\title{
Free field representation of the $\mathrm{ZF}$ algebra of the $\mathrm{SU}(\mathrm{N}) \times \mathrm{SU}(\mathrm{N}) \mathrm{PCF}$ model
}

\author{
Sergey Frolov刚 \\ School of Mathematics and Hamilton Mathematics Institute, \\ Trinity College, Dublin 2, Ireland
}

\begin{abstract}
A free field representation of the Zamolodchikov-Faddeev algebra of the $\mathrm{SU}(\mathrm{N}) \times \mathrm{SU}(\mathrm{N})$ Principal Chiral Field model is constructed, and used to derive an integral representation for form factors of a multi-parameter family of exponential fields.

This paper is a tribute to the memory of Prof. Petr Kulish.
\end{abstract}

* Correspondent fellow at Steklov Mathematical Institute, Moscow.

$\dagger$ email: frolovs@maths.tcd.ie 


\section{Contents}

1 Introduction 2

2 Generalities 4

2.1 The S-matrix of the model . . . . . . . . . . . . . . 4

2.2 Form factors . . . . . . . . . . . . . . . . 6

2.3 Free fields and basic vertex operators . . . . . . . . . . . . . 7

3 Free field representation 9

3.1 Charges and free fields in the large $N$ limit . . . . . . . . . . . . . 9 9

3.2 Ansatz for ZF operators and charges . . . . . . . . . . . . . 11

3.3 List of Green's functions . . . . . . . . . . . . . . . . . . . 12

3.4 The angular Hamiltonian . . . . . . . . . . . . . . . . . . . . . 14

3.5 Commutativity relations . . . . . . . . . . . . . 15

4 The ZF algebra 16

4.1 The ZF relations . . . . . . . . . . . . . . . . . . . . 16

4.2 Integration contours and the ZF operators $Z_{2 \dot{1}}, Z_{1 \dot{2}}, Z_{2 \dot{2}} \ldots \ldots \ldots$

4.3 The ZF algebra for $Z_{11}, Z_{21}, Z_{12}, Z_{22} \ldots \ldots \ldots \ldots$

5 Bound states $\quad 20$

6 Form factors

7 Conclusion 28

A Green's functions derivation 29

B Constraints and elementary free fields 33

C ZF operators $Z_{2 \dot{1}}, Z_{1 \dot{2}}, Z_{2 \dot{2}}$

D Traces of vertex operators 40

\section{Introduction}

Form factors of a two-dimensional quantum field theory model are matrix elements between the vacuum, $|v a c\rangle$, and in-states

$$
F_{K_{1} \ldots K_{n}}\left(\theta_{1}, \ldots, \theta_{n}\right)=\left\langle v a c|O(0)| \theta_{1}, \ldots, \theta_{n}\right\rangle_{K_{1} \ldots K_{n}}^{(i n)}
$$

where $K_{i}$ is a flavour index of the $i$-th particle, and $\theta_{i}$ is its rapidity variable related to its energy and momentum by $E_{i}=m_{i} \cosh \theta_{i}, p_{i}=m_{i} \sinh \theta_{i}$. In a crossing invariant 
theory one can express a generic matrix element, $\langle$ out $|O(x)| i n\rangle$, of a local operator $O$ between in- and out-states in terms of the analytically continued form factors.

The form factors of integrable two-dimensional relativistic models satisfy certain axioms [1]-[3] which have been solved for some models, see e.g. [3]-[11]. Finding a solution to the axioms is highly nontrivial and requires an extensive use of the form factors' analytic properties. An important observation by Lukyanov [12] (following the ideas in [13]) is that for a given model form factors can be found by constructing a free field representation of its Zamolodchikov-Faddeev (ZF) algebra [14, 15]. Lukyanov's approach has been successfully applied to several models [12], [16]-[27], in particular in [26. 27] to the $\mathrm{SU}(\mathrm{N})$ Gross-Neveu (GN) model [28]. An advantage of this approach is that the analytic properties of form factors follow from a free field representation. This might be helpful for nonrelativistic but crossing invariant models where analytic properties of form factors are not completely understood. An important example of such a model is provided by the $\mathrm{AdS}_{5} \times \mathrm{S}^{5}$ superstring sigma model in the light-cone gauge [29]. Even though most of the form factors axioms can be generalised to the case [30] no solution has been found yet because the analytic properties of the $\operatorname{Ad}_{5} \times \mathrm{S}^{5}$ form factors are unknown. One may hope that Lukyanov's approach might be more efficient in the $\mathrm{AdS}_{5} \times \mathrm{S}^{5}$ case.

Another complication of the $\mathrm{AdS}_{5} \times \mathrm{S}^{5}$ model is that its symmetry algebra is a sum of two algebras (which are in addition centrally-extended super Lie algebras sharing a central element). The only model of such a type for which a free field representation has been constructed is the two-parameter family of integrable models (the SS model) [32]. This representation was found by Fateev and Lashkevich [19]. A generalisation of their results to other models is not straightforward.

The goal of this paper is to extend Lukyanov's approach to the $\mathrm{SU}(\mathrm{N}) \times \mathrm{SU}(\mathrm{N})$ Principal Chiral Field (PCF) model. The symmetry algebra of the model is obviously $\mathfrak{s u}(N) \oplus \mathfrak{s u}(N)$. The "elementary" particles of the model transform in the rank-1 bifundamental representation of $\mathrm{SU}(\mathrm{N}) \times \mathrm{SU}(\mathrm{N})$, and they form $r$-particle bound states transforming in the rank- $r$ bi-fundamental representations of $\mathrm{SU}(\mathrm{N}) \times \mathrm{SU}(\mathrm{N})$ [33]. The exact S-matrix of the PCF model up to a CDD factor [34] is a direct product of the S-matrices of the chiral GN model [35]-[39], and can be found from the usual requirements of unitarity, analyticity, crossing symmetry and the bound state structure [33]. Similarly to the chiral GN model [36], anti-particles of elementary particles are bound states of $N-1$ elementary particles, and in general anti-particles of rank- $r$ particles are rank- $(N-r)$ particles.

Nothing is known about form factors of the PCF model for finite $N \geq 3$ except the two-particle form factor of the current operator found in [40]. At infinite $N$ multiparticle form factors of the renormalised field operator were found in [41, and those of the current and energy-momentum tensor operators in [42, 43]. Since up to a twist the

\footnotetext{
${ }^{1}$ Notice that the axioms for a cubic light-cone string field theory vertex [31] are very similar to the form factors axioms.
} 
$\mathrm{SU}(2) \times \mathrm{SU}(2)=\mathrm{O}(4)$ model can be obtained from the SS model in a special limit $p_{1}, p_{2} \rightarrow$ $\infty$, much more is known about the $N=2$ case. The form factors of the SS model for a large class of local operators which includes the $U(1)$ currents and energy-momentum tensor where determined in [4] by solving the form factor axioms, and those for a 3parameter family of exponential fields in [32] by constructing a free field representation of the ZF algebra.

In this paper a free field representation of the $\mathrm{ZF}$ algebra of the $\mathrm{SU}(\mathrm{N}) \times \mathrm{SU}(\mathrm{N})$ PCF model for elementary particles and their bound states is constructed. It can be used to derive an integral representation for form factors of a multi-parameter family of exponential fields through Lukyanov's trace formula [12]. The determination of the precise form of the exponential fields and their relation to the fields which appear in the Lagrangian of the PCF model is outside the scope of the paper. For $N=2$ the free field representation and the integral representation should be equivalent to those found by Fateev and Lashkevich [19]. However, the $\mathrm{O}(4)$ limit of their representation is subtle, and in this paper a proof of the equivalence is not attempted.

The outline of the paper is as follows. In section 2 the properties of the scattering matrix, and the particles content of the PCF model are reviewed. Here, the general idea of the free field representation approach to form factors is also explained. In section 3 the construction of a representation of the extended ZF algebra and angular Hamiltonian for the PCF model is considered, Green's functions and relations between free fields are listed, and the main properties of the representation are discussed. Next, it is proven in section 4 that the vertex operators constructed in section 3 indeed satisfy the $\mathrm{ZF}$ algebra relations. Then, the derivation of the highest weight bound state vertex operators and bound states is performed in section 5. Finally, form factors are discussed in section 6, where general formulae for constructing form factors are established. In several appendices the necessary functions are collected and the derivations of some results stated in the main text are presented.

\section{Generalities}

\subsection{The S-matrix of the model}

The spectrum of particles of the $\mathrm{SU}(\mathrm{N}) \times \mathrm{SU}(\mathrm{N})$ PCF model consists of elementary particles of mass $m$ transforming in the rank- 1 bi-fundamental representation of $\mathrm{SU}(\mathrm{N}) \times \mathrm{SU}(\mathrm{N})$, and their $r$-particle bound states of mass $m_{r}=m \sin \frac{\pi r}{N} / \sin \frac{\pi}{N}$ transforming in the rank $r=2, \ldots, N-1$ bi-fundamental representation of $\mathrm{SU}(\mathrm{N}) \times \mathrm{SU}(\mathrm{N})$. A rank- $r$ particle with rapidity $\theta$ is created by a ZF operator $\mathcal{A}_{K \dot{K}}^{\dagger}(\theta)$, and annihilated by $\mathcal{A}^{K \dot{K}}(\theta)$ where $K=\left(k_{1}, \ldots, k_{r}\right)$ and $\dot{K}=\left(\dot{k}_{1}, \ldots, \dot{k}_{r}\right)$ have integer-valued components ordered as $1 \leq k_{1}<k_{2}<\cdots<k_{r} \leq N$, and $\dot{1} \leq \dot{k}_{1}<\dot{k}_{2}<\cdots<\dot{k}_{r} \leq \dot{N}$. The left and right $\mathrm{SU}(\mathrm{N})$ groups act on the undotted and dotted indices respectively. 
The S-matrix for the elementary particles of the PCF model is [33]

$$
\begin{gathered}
\mathbb{S}^{\mathrm{PCF}}(\theta)=\chi_{C D D}(\theta) \cdot S(\theta) \mathbb{R}(\theta) \otimes S(\theta) \mathbb{R}(\theta)=S_{P C F}(\theta) \mathbb{R}(\theta) \otimes \mathbb{R}(\theta), \\
S(\theta)=-\frac{\Gamma\left(\frac{i \theta}{2 \pi}\right) \Gamma\left(\frac{1}{N}-\frac{i \theta}{2 \pi}\right)}{\Gamma\left(-\frac{i \theta}{2 \pi}\right) \Gamma\left(\frac{1}{N}+\frac{i \theta}{2 \pi}\right)}, \quad \chi_{C D D}(\theta)=\frac{\sinh \left(\frac{\theta}{2}+\frac{i \pi}{N}\right)}{\sinh \left(\frac{\theta}{2}-\frac{i \pi}{N}\right)} .
\end{gathered}
$$

Here the standard $\mathrm{SU}(\mathrm{N})$-invariant R-matrix is

$$
\mathbb{R}(\theta)=\frac{\theta \mathbb{I}-\frac{2 \pi i}{N} \mathbb{P}}{\theta-\frac{2 \pi i}{N}}=\mathbb{P}_{s}+\frac{\theta+\frac{2 \pi i}{N}}{\theta-\frac{2 \pi i}{N}} \mathbb{P}_{a}
$$

where $\mathbb{P}$ is the permutation operator and $\mathbb{P}_{s}=\frac{1}{2}(\mathbb{I}+\mathbb{P}), \mathbb{P}_{a}=\frac{1}{2}(\mathbb{I}-\mathbb{P})$, are the projection operators onto the symmetric and antisymmetric parts of the tensor product of two fundamental representations. The $\mathrm{S}$-matrix $\mathbb{S}^{\mathrm{PCF}}(\theta)$ has a single pole at $\theta=\frac{2 \pi i}{N}$ due to the pole of $\mathbb{R}(\theta)$ in the antisymmetric part and the zero of $S_{P C F}(\theta)$ at $\theta=\frac{2 \pi i}{N}$ which leads to the existence of the rank- $r$ bound states. The $(N-1)$-particle bound states are identified with anti-particles of the elementary particles. In general a rank- $r$ and a rank$(N-r)$ particles created by $\mathcal{A}_{K \dot{K}}^{\dagger}$ and $\mathcal{A}_{\bar{K} \bar{K}}^{\dagger}$ form a particle-antiparticle pair if $\bar{K}$ and $\overline{\dot{K}}$ are such that $K \cup \bar{K}=\mathcal{P}(1,2, \ldots, N)$ and $\dot{K} \cup \bar{K}=\dot{\mathcal{P}}(\dot{1}, \dot{2}, \ldots, \dot{N})$ where $\mathcal{P}$ and $\dot{\mathcal{P}}$ are some permutations of $1,2, \ldots N$, and $\dot{1}, \dot{2}, \ldots, \dot{N}$, respectively. In what follows in such a pair a bound state of smaller rank (that is $r<N / 2$ ) is considered as a particle. If $N$ is even, $N=2 p$, then a bound state with the label $K=\left(1, k_{2}, \ldots, k_{p}\right)$ is considered as a particle. The ZF operators can be normalised in such a way that for a particle $\mathcal{A}_{K \dot{K}}^{\dagger}$ and antiparticle $\mathcal{A}_{L \dot{L}}^{\dagger}$ the charge conjugation matrix $C_{K \dot{K}, L \dot{L}}=\epsilon_{K L} \epsilon_{\dot{K} \dot{L}}$ where $\epsilon_{K L} \equiv \epsilon_{i_{1} \ldots i_{N}}$ is skew-symmetric, and $\epsilon_{1 \ldots N}=1$, and similarly for $\epsilon_{\dot{K} \dot{L}}$. The S-matrices of the bound states are obtained from the S-matrix for elementary particles by the fusion procedure. The creation and annihilation operators satisfy the ZF algebra [14, 15].

Up to a CDD factor the $\mathrm{SU}(\mathrm{N}) \times \mathrm{SU}(\mathrm{N}) \mathrm{PCF}$ model can be thought of as the tensor product of two chiral SU(N) Gross-Neveu models due to the following relation between their S-matrices for the elementary particles

$$
\mathbb{S}^{\mathrm{PCF}}(\theta)=\left(\mathbb{S}^{G N}(\theta) \otimes \mathbb{S}^{G N}(\theta)\right) \chi_{C D D}(-\theta)
$$

where

$$
\begin{gathered}
\mathbb{S}^{\mathrm{GN}}(\theta)=S_{G N}(\theta) \mathbb{R}(\theta), \\
S_{G N}(\theta)=S(\theta) \chi_{C D D}(\theta)=\frac{\Gamma\left(\frac{i \theta}{2 \pi}\right) \Gamma\left(\frac{N-1}{N}-\frac{i \theta}{2 \pi}\right)}{\Gamma\left(-\frac{i \theta}{2 \pi}\right) \Gamma\left(\frac{N-1}{N}+\frac{i \theta}{2 \pi}\right)} .
\end{gathered}
$$

The Gross-Neveu S-matrix satisfies the crossing symmetry condition

$$
\prod_{k=-\frac{N-1}{2}}^{\frac{N-1}{2}} S_{G N}\left(\theta+\frac{2 \pi i}{N} k\right)=(-1)^{N-1} \frac{\theta-i \pi \frac{N-1}{N}}{\theta+i \pi \frac{N-1}{N}}
$$


and has the large $\theta$ asymptotics $S( \pm \infty)=e^{\mp i \pi \frac{N-1}{N}}$. It admits the nice integral form

$$
S_{G N}(\theta)=\exp \left(-2 i \int_{0}^{\infty} \frac{d t}{t} \frac{e^{\frac{\pi t}{N}} \sinh \frac{(N-1) \pi t}{N}}{\sinh \pi t} \sin \theta t\right)
$$

Writing an integral representation of the CDD factor, one finds

$$
\chi_{C D D}(\theta)=-\exp \left(-2 i \int_{0}^{\infty} \frac{d t}{t} \frac{\sinh \pi t\left(1-\frac{2}{N}\right)}{\sinh \pi t} \sin \theta t\right)
$$

and therefore

$$
S_{P C F}(\theta)=S_{1 \mathrm{i} 1 \mathrm{i}}^{1 \mathrm{i} \dot{1}}(\theta)=\exp \left(-4 i \int_{0}^{\infty} \frac{d t}{t} \frac{\sinh \frac{\pi t}{N} \sinh \left(\frac{(N-1) \pi t}{N}\right)}{\sinh \pi t} \sin \theta t\right)=\frac{g_{P C F}(-\theta)}{g_{P C F}(\theta)} .
$$

Here

$$
g_{P C F}(\theta)=\frac{\Gamma\left(\frac{i \theta}{2 \pi}-\frac{1}{N}+1\right) \Gamma\left(\frac{i \theta}{2 \pi}+\frac{1}{N}\right)}{\Gamma\left(\frac{i \theta}{2 \pi}\right) \Gamma\left(\frac{i \theta}{2 \pi}+1\right)}
$$

is the Green's function which will appear in the free field representation of the PCF model to be constructed in this paper.

\subsection{Form factors}

The ZF operators are used to create the in- and out-states as follows

$$
\begin{array}{ll}
\left|\theta_{1}, \theta_{2}, \cdots, \theta_{n}\right\rangle_{\mathbb{I}_{1}, \ldots, \mathbb{I}_{n}}^{(i n)}=\mathcal{A}_{\mathbb{I}_{n}}^{\dagger}\left(\theta_{n}\right) \cdots \mathcal{A}_{\mathbb{I}_{1}}^{\dagger}\left(\theta_{1}\right)|v a c\rangle, & \theta_{1}<\theta_{2}<\cdots<\theta_{n}, \\
\left|\theta_{1}, \theta_{2}, \cdots, \theta_{n}\right\rangle_{\mathbb{I}_{1}, \ldots, \mathbb{I}_{n}}^{(\text {out })}=\mathcal{A}_{\mathbb{I}_{1}}^{\dagger}\left(\theta_{1}\right) \cdots \mathcal{A}_{\mathbb{I}_{n}}^{\dagger}\left(\theta_{n}\right)|v a c\rangle, & \theta_{1}<\theta_{2}<\cdots<\theta_{n},
\end{array}
$$

where $\mathbb{I}$ is a multi-index $\mathbb{I} \equiv I \dot{I}$, and the vacuum state $|v a c\rangle$ is annihilated by $\mathcal{A}^{\mathbb{I}}(\theta)$, and has the unit norm, $\langle v a c \mid v a c\rangle=1$.

Thus, form factors (1.1) of a local operator $O(x)$ can be written as

$$
F_{\mathbb{I}_{1} \ldots \mathbb{I}_{n}}\left(\theta_{1}, \ldots, \theta_{n}\right)=\left\langle\operatorname{vac}\left|O(0) \mathcal{A}_{\mathbb{I}_{n}}^{\dagger}\left(\theta_{n}\right) \cdots \mathcal{A}_{\mathbb{I}_{1}}^{\dagger}\left(\theta_{1}\right)\right| v a c\right\rangle .
$$

According to Lukyanov [12], the determination of form factors can be reduced to the problem of finding a representation of a so-called extended $\mathrm{ZF}$ algebra which is generated by vertex operators $Z_{\mathbb{I}}(\theta)$, the angular Hamiltonian $\mathbb{K}$, and the central elements $\Omega_{\mathbb{I}}$ obeying the defining relations

$$
\begin{aligned}
Z_{\mathbb{I}}\left(\theta_{1}\right) Z_{\mathbb{J}}\left(\theta_{2}\right) & =Z_{\mathbb{L}}\left(\theta_{2}\right) Z_{\mathbb{K}}\left(\theta_{1}\right) S_{\mathbb{I} \mathbb{I}}^{\mathbb{K} \mathbb{L}}\left(\theta_{12}\right), \\
Z_{\mathbb{I}}\left(\theta_{1}\right) Z_{\mathbb{J}}\left(\theta_{2}\right) & =-\frac{i C_{\mathbb{I}}}{\theta_{12}-i \pi}+\mathcal{O}(1), \quad \theta_{12} \rightarrow i \pi, \\
\frac{d}{d \theta} Z_{\mathbb{I}}(\theta) & =-\left[\mathbb{K}, Z_{\mathbb{I}}(\theta)\right]-i \Omega_{\mathbb{I}} Z_{\mathbb{I}}(\theta), \\
Z_{\mathbb{I}}\left(\theta^{\prime}+i \mathfrak{u}_{+}\right) Z_{\mathbb{J}}\left(\theta-i \mathfrak{u}_{-}\right) & =\frac{i}{\theta^{\prime}-\theta} \sum_{\mathbb{K} \in \mathcal{K}} \Gamma_{\mathbb{I} \mathbb{J}}^{\mathbb{K}} Z_{\mathbb{K}}(\theta)+\mathcal{O}(1), \quad \theta^{\prime} \rightarrow \theta,
\end{aligned}
$$


where the notation $\theta_{i j} \equiv \theta_{i}-\theta_{j}$ is used. The bootstrap conditions 2.16) are required if the particles $\mathcal{A}_{\mathbb{K}}^{\dagger}$ of the same mass with $\mathbb{K} \in \mathcal{K}$ are bound states of particles $\mathcal{A}_{\mathbb{I}}^{\dagger}$ and $\mathcal{A}_{\mathbb{J}}^{\dagger}$ with $\mathbb{I} \in \mathcal{I}$ and $\mathbb{J} \in \mathcal{J}$. The mass of the bound state $\mathcal{A}_{\mathbb{K}}^{\dagger}$ is equal to $m_{\mathbb{K}}=$ $m_{\mathbb{I}} \cos \mathfrak{u}_{+}+m_{\mathbb{J}} \cos \mathfrak{u}_{-}$where $\mathfrak{u}_{ \pm}$are found from the equations

$$
\mathfrak{u}_{+}+\mathfrak{u}_{-}=\mathfrak{u}_{\mathbb{I} \mathbb{J}}^{\mathbb{K}}, \quad m_{\mathbb{I}} \sin \mathfrak{u}_{+}=m_{\mathbb{J}} \sin \mathfrak{u}_{-} .
$$

The scattering matrix of the particles $\mathcal{A}_{\mathbb{I}}^{\dagger}$ and $\mathcal{A}_{\mathbb{J}}^{\dagger}$ must have a simple pole at $\theta=i \mathfrak{u}_{\mathbb{I}}^{\mathbb{K}}$. $\Gamma_{\mathbb{I} \mathbb{J}}^{\mathbb{K}}$ are some constants, and the relations $(2.16)$ can be inverted and used to derive the vertex operators for the bound states from the vertex operators for elementary particles.

Notice that due to 2.13) and (2.14), $Z_{\mathbb{I}}(\theta)$ and $C^{\mathbb{I} \mathbb{J}} Z_{\mathbb{J}}(\theta+i \pi), C^{\mathbb{I}} C_{\mathbb{J}}=\delta_{\mathbb{K}}^{\mathbb{I}}$ can be thought as representing the ZF creation and annihilation operators, respectively:2

An important observation of Lukyanov is that any representation $\pi_{O}$ of the extended $\mathrm{ZF}$ algebra corresponds to a local operator $O$, and the form factors 2.12 of this operator are given by the formula

$$
F_{\mathbb{I}_{1} \ldots \mathbb{I}_{n}}^{O}\left(\theta_{1}, \ldots, \theta_{n}\right)=\mathcal{N}_{O} \frac{\operatorname{Tr}_{\pi_{O}}\left[e^{2 \pi i \mathbb{K}} Z_{\mathbb{I}_{n}}\left(\theta_{n}\right) \cdots Z_{\mathbb{I}_{1}}\left(\theta_{1}\right)\right]}{\operatorname{Tr}_{\pi_{O}}\left[e^{2 \pi i \mathbb{K}}\right]},
$$

where the normalisation constant $\mathcal{N}_{O}$ depends only on the local operator $O$ and has to be fixed by other means. It can be shown that if (2.18) satisfies the necessary analyticity properties, then the form factor axioms follow from the cyclicity of the trace and the extended ZF algebra.

In addition, if one finds a linear operator $\Lambda(\tilde{O})$ acting in $\pi_{O}$ which satisfies

$$
e^{\theta \mathbb{K}} \Lambda(\tilde{O}) e^{-\theta \mathbb{K}}=e^{\theta s(\tilde{O})} \Lambda(\tilde{O}), \quad \Lambda(\tilde{O}) Z_{\mathbb{I}}(\theta)=e^{2 \pi i \Omega(\tilde{O}, I)} Z_{\mathbb{I}}(\theta) \Lambda(\tilde{O}),
$$

then it corresponds to some local operator $\tilde{O}(x)$ with the spin $s(\tilde{O})$, and the form factors of the operator : $\tilde{O}(x) O(x)$ : are given by

$$
F_{\mathbb{I}_{1} \ldots \mathbb{I}_{n}}^{\tilde{O} O}\left(\theta_{1}, \ldots, \theta_{n}\right)=\mathcal{N}_{\tilde{O} O} \frac{\operatorname{Tr}_{\pi_{O}}\left[e^{2 \pi i \mathbb{K}} \Lambda(\tilde{O}) Z_{\mathbb{I}_{n}}\left(\theta_{n}\right) \cdots Z_{\mathbb{I}_{1}}\left(\theta_{1}\right)\right]}{\operatorname{Tr}_{\pi_{O}}\left[e^{2 \pi i \mathbb{K}}\right]}
$$

Notice that $\Omega(\tilde{O}, I)$ appears in $(2.19)$ if the particle $\mathcal{A}_{\mathbb{I}}^{\dagger}$ has nontrivial statistics with respect to $\tilde{O}(x)$.

\subsection{Free fields and basic vertex operators}

The second important result by [12] is that for many models the extended ZF algebra can be realised in terms of free bosons. In what follows free fields $\phi_{\mu}(\theta)$ which satisfy the following relations

$$
\left[\phi_{\mu}\left(\theta_{1}\right), \phi_{\nu}\left(\theta_{2}\right)\right]=\ln S_{\nu \mu}\left(\theta_{21}\right), \quad\left\langle\phi_{\mu}\left(\theta_{1}\right) \phi_{\nu}\left(\theta_{2}\right)\right\rangle=-\ln g_{\nu \mu}\left(\theta_{21}\right),
$$

\footnotetext{
${ }^{2}$ For the $\mathrm{SU}(2 \mathrm{p})$ chiral GN model the relations $(2.14)$ are modified by replacing $C_{\mathbb{I} \mathbb{J}}$ with $C_{\mathbb{I J}} \Gamma$ where $\Gamma$ is an auxiliary element satisfying $\Gamma^{2}=i d$ which (anti-)commutes with $Z_{I}$ [27].
} 
are used. The S-matrices $S_{\mu \nu}$ and Green's functions $g_{\mu \nu}$ are related to each other as

$$
S_{\mu \nu}(\theta)=\frac{g_{\nu \mu}(-\theta)}{g_{\mu \nu}(\theta)}=\frac{1}{S_{\nu \mu}(-\theta)} .
$$

The fields $\phi_{\mu}$ are used to construct the basic vertex operators

$$
V_{\mu}(\theta)=: e^{i \phi_{\mu}(\theta)}: \equiv e^{i \phi_{\mu}(\theta)}
$$

which obey the following relations

$$
V_{\mu}\left(\theta_{1}\right) V_{\nu}\left(\theta_{2}\right)=g_{\nu \mu}\left(\theta_{21}\right): V_{\mu}\left(\theta_{1}\right) V_{\nu}\left(\theta_{2}\right):, \quad V_{\mu}\left(\theta_{1}\right) V_{\nu}\left(\theta_{2}\right)=S_{\mu \nu}\left(\theta_{12}\right) V_{\nu}\left(\theta_{2}\right) V_{\mu}\left(\theta_{1}\right) .
$$

The free fields can be written in the form

$$
\phi_{\mu}(\theta)=Q_{\mu}+\int_{-\infty}^{\infty} \frac{d t}{i t} A_{\mu}(t) e^{i \theta t}
$$

where the creation $A_{\mu}(-t), t>0$ and annihilation operators $A_{\mu}(t)|0\rangle=0, t>0$ have the commutation relations

$$
\left[A_{\mu}(t), A_{\nu}\left(t^{\prime}\right)\right]=t f_{\nu \mu}(t) \delta\left(t+t^{\prime}\right), \quad f_{\mu \nu}(-t)=f_{\nu \mu}(t) .
$$

The zero mode operators $Q_{\mu}$ commute with $A_{\nu}(t)$, and are introduced to guarantee that the ZF operators have correct eigenvalues with respect to the Cartan generators $P_{\mu}$ which annihilate the vacuum: $P_{\mu}|0\rangle=0$. Their commutation relations will not be important for this paper.

The S-matrices $S_{\mu \nu}$ and Green's functions $g_{\mu \nu}$ are related to $f_{\mu \nu}$ as follows

$$
S_{\mu \nu}(\theta)=\exp \left(\int_{-\infty}^{\infty} \frac{d t}{t} f_{\mu \nu}(t) e^{-i \theta t}\right), \quad g_{\mu \nu}(\theta)=\exp \left(-\int_{0}^{\infty} \frac{d t}{t} f_{\mu \nu}(t) e^{-i \theta t}\right),
$$

where it is assumed that the integral representation for $S_{\mu \nu}(\theta)$ is well-defined for some $\theta$ 's. If it is not defined then one should analytically continue Green's functions $g_{\mu \nu}(\theta)$ and $g_{\nu \mu}(-\theta)$ to a common domain and calculate their ratio to find $S_{\mu \nu}(\theta)$.

The annihilation and creation parts of $\phi_{\mu}(\theta)$ will be denoted as follows

$$
\phi_{\mu}^{-}(\theta)=\int_{0}^{\infty} \frac{d t}{i t} A_{\mu}(t) e^{i \theta t}, \quad \phi_{\mu}^{+}(\theta)=\int_{-\infty}^{0} \frac{d t}{i t} A_{\mu}(t) e^{i \theta t}=-\int_{0}^{\infty} \frac{d t}{i t} A_{\mu}(-t) e^{-i \theta t} .
$$

It is also important to mention that the integrals of the form

$$
\int_{0}^{\infty} d t F(t)
$$

will be always understood as [44]

$$
\int_{0}^{\infty} d t F(t) \equiv \int_{C_{0}} \frac{d t}{2 \pi i} F(t) \ln (-t),
$$

where the integration contour $C_{0}$ is shown in Figure 1 . 


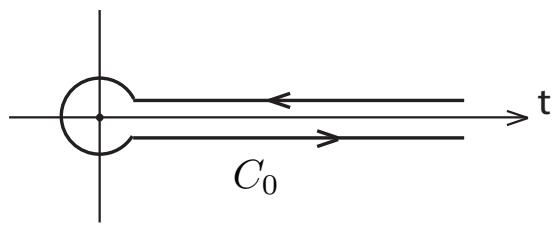

Figure 1: The integration contour $C_{0}$ in the integral $\int_{C_{0}} \frac{d t}{2 \pi i} F(t) \ln (-t)$.

\section{Free field representation}

The ZF operators for the elementary particles of the PCF model are $\mathbb{A}_{k \dot{k}}^{\dagger}, k=1, \ldots, N$, $\dot{k}=\dot{1}, \ldots, \dot{N}]^{3}$ The $\mathrm{SU}(\mathrm{N}) \times \mathrm{SU}(\mathrm{N})$ charges $\mathbb{R}_{b}{ }^{a}$ and $\mathbb{R}_{\dot{b}}{ }^{\dot{a}}$ act on the ZF operators as follows

$$
\mathbb{R}_{b}^{a} \mathbb{A}_{k \dot{k}}^{\dagger}-\mathbb{A}_{k \dot{k}}^{\dagger} \mathbb{R}_{b}^{a}=\delta_{k}^{a} \mathbb{A}_{b \dot{k}}^{\dagger}, \quad \dot{\mathbb{R}}_{\dot{b}}^{\dot{a}} \mathbb{A}_{k \dot{k}}^{\dagger}-\mathbb{A}_{k \dot{k}}^{\dagger} \dot{\mathbb{R}}_{\dot{b}}^{\dot{a}}=\delta_{\dot{k}}^{\dot{a}} \mathbb{A}_{k \dot{b}}^{\dagger}
$$

It is natural to use $\mathbb{A}_{1 \mathrm{i}}^{\dagger}$ as the highest weight operator, and charges $\mathbb{J}_{k} \equiv \mathbb{R}_{k+1}{ }^{k}$ and $\dot{\mathbb{J}}_{\dot{k}} \equiv \dot{\mathbb{R}}_{\dot{k}+\dot{1}}{ }^{\dot{k}}$ as the lowering operators which produce all elementary particles ZF operators from $\mathbb{A}_{1 i}^{\dagger}$.

\subsection{Charges and free fields in the large $N$ limit}

To motivate the free field representation which is described in the next subsection it is useful to consider the large $N$ limit. In this limit the S-matrix goes to the identity matrix, and the ZF algebra for the ZF operators becomes the usual Heisenberg algebra of the annihilation and creation operators $a^{m \dot{m}}$ and $a_{n \dot{n}}^{\dagger}$ whose nontrivial commutation relations are

$$
a^{m \dot{m}}\left(\theta_{1}\right) a_{n \dot{n}}^{\dagger}\left(\theta_{2}\right)-a_{n \dot{n}}^{\dagger}\left(\theta_{2}\right) a^{m \dot{m}}\left(\theta_{1}\right)=\delta_{n}^{m} \delta_{\dot{n}}^{\dot{m}} \delta\left(\theta_{12}\right) .
$$

These operators and relations depend on the rapidities but the dependence will be often omitted to simplify the expressions below. The charges of the two copies of $\mathfrak{s u}(N)$ 's are built from the operators

$$
V_{m \dot{r}}^{n} \equiv a_{m \dot{r}}^{\dagger} a^{n \dot{r}}, \quad \dot{V}_{\dot{m} r}^{\dot{n}} \equiv a_{r \dot{m}}^{\dagger} a^{r \dot{n}}
$$

These operators satisfy the commutation relations

$$
\begin{aligned}
& V_{m \dot{r}}^{n} V_{k \dot{q}}^{l}-V_{k \dot{q}}^{l} V_{m \dot{r}}^{n}=\delta_{k}^{n} \delta_{\dot{q} \dot{r}} V_{m \dot{r}}^{l}+\delta_{m}^{l} \delta_{\dot{q} \dot{r}} V_{k \dot{r}}^{n}, \\
& \dot{V}_{\dot{m} r}^{\dot{n}} \dot{V}_{\dot{k} q}^{l}-\dot{V}_{\dot{k} q}^{i} \dot{V}_{\dot{m} r}^{\dot{n}}=\delta_{\dot{k}}^{\dot{n}} \delta_{q r} \dot{V}_{\dot{m} r}^{i}+\delta_{\dot{m}}^{l} \delta_{q r} \dot{V}_{\dot{k} r}^{\dot{n}}, \\
& V_{m \dot{r}}^{n} \dot{V}_{\dot{k} q}^{l}-\dot{V}_{\dot{k} q}^{l} V_{m \dot{r}}^{n}=\left(\delta_{q}^{n} \delta_{\dot{k}}^{\dot{r}}-\delta_{m}^{q} \delta_{\dot{r}}^{l}\right) a_{m \dot{k}}^{\dagger} a^{n \dot{l}} .
\end{aligned}
$$

\footnotetext{
${ }^{3}$ The dotted and undotted indices are obviously different $\dot{k} \neq k$. However, in some formulae they are identified which will be clearly stressed out.
} 
One sees from the last relation that the operators

$$
R_{m}^{n} \equiv \sum_{\dot{r}=1}^{\dot{N}} \int d \alpha V_{m \dot{r}}^{n}(\alpha), \quad \dot{R}_{\dot{k}}^{i} \equiv \sum_{q=1}^{N} \int d \alpha \dot{V}_{\dot{k} q}^{i}(\alpha),
$$

commute

$$
R_{m}^{n} \dot{R}_{\dot{k}}^{i}-\dot{R}_{\dot{k}}^{i} R_{m}^{n}=0,
$$

and it is not difficult to check that they form the $\mathfrak{u}(N) \oplus \mathfrak{u}(N)$ algebra.

The charges $R_{k+1}{ }^{k}$ and $R_{\dot{k}+\dot{1}}{ }^{\dot{k}}$ are linear combinations of $V_{k+1, \dot{r}}^{k}$ and $\dot{V}_{\dot{k}+\dot{1}, r}^{\dot{k}}$ whose nontrivial relations are

$$
\begin{gathered}
V_{k+1, \dot{r}}^{k} V_{n \dot{q}}^{n-1}=\delta_{k n} \delta_{\dot{r} \dot{q}} V_{k+1, \dot{r}}^{k-1}+: V_{k+1, \dot{r}}^{k} V_{n \dot{r}}^{n-1}:, \\
\dot{V}_{\dot{k}+\mathrm{i}, r}^{\dot{k}} \dot{V}_{\dot{n} q}^{\dot{n}-\dot{1}}=\delta_{\dot{k} \dot{n}} \delta_{r q} \dot{V}_{\dot{k}+\dot{1}, r}^{\dot{k}-\dot{1}}+: \dot{V}_{\dot{k}+\dot{1}, r}^{\dot{k}} \dot{V}_{\dot{n} q}^{\dot{n}-1}:, \\
V_{k+1, \dot{r}}^{k} \dot{V}_{\dot{n} q}^{\dot{n}-\dot{1}}=\delta_{\dot{r} \dot{n}} \delta_{k q} a_{k+1, \dot{n}}^{\dagger} a^{k, \dot{n}-\dot{1}}+: V_{k+1, \dot{r}}^{k} \dot{V}_{\dot{n} q}^{\dot{n}-\dot{1}}:, \\
\dot{V}_{\dot{n} q}^{\dot{n}-\dot{1}} V_{k+1, \dot{r}}^{k}=\delta_{\dot{r}, \dot{n}-\dot{1}} \delta_{k+1, q} a_{k+1, \dot{n}}^{\dagger} a^{k, \dot{n}-\dot{1}}+: \dot{V}_{\dot{n} q}^{\dot{n}-\dot{1}} V_{k+1, \dot{r}}^{k}:,
\end{gathered}
$$

where Kronecker's deltas are multiplied by Dirac's delta of the difference of the rapidities the operators depend on.

In terms of free fields it is therefore reasonable to assume that the charges $\mathbb{J}_{k}$ and $\dot{\mathbb{J}}_{k}$ are linear combinations of the following vertex operators

$$
V_{k+1, \dot{r}}^{k}(\alpha) \sim e^{i \phi_{k \dot{r}}(\alpha)}, \quad \dot{V}_{\dot{k}+\dot{1}, r}^{\dot{k}}(\alpha) \sim e^{i \dot{\phi}_{k_{r}}(\alpha)} .
$$

Moreover, it is natural to expect that a delta-function term in the product $V_{\mu}\left(\theta_{1}\right) V_{\nu}\left(\theta_{2}\right)$ of two $V$ 's in (3.9-3.12) implies that the corresponding Green's function $g_{\nu \mu}\left(\theta_{21}\right)$ has a pole at $\theta_{21}=0$. On the other hand even if the product of two $V$ 's in 3.93 .12 is regular it does not mean that the corresponding Green's function is equal to 1 because these relations are only valid in the large $N$ limit.

Thus, the appearance of the delta-function terms in the products

$$
V_{k+1, \dot{r}}^{k} V_{k \dot{r}}^{k-1}, \quad \dot{V}_{\dot{k}+\dot{1}, r}^{\dot{k}} \dot{V}_{\dot{k} r}^{\dot{k}-\dot{1}}, \quad V_{k+1, \dot{n}+\dot{1}}^{k} \dot{V}_{\dot{n}+\dot{1}, k}^{\dot{n}} \text { and } \dot{V}_{\dot{n}+1, k+1}^{\dot{n}} V_{k+1, \dot{n}}^{k}
$$

implies that the Green's functions

$$
g_{k-1, \dot{r} \mid k \dot{r}}(\theta), \quad g_{\dot{k}-\dot{i}, r \mid \dot{k} r}(\theta), \quad g_{\dot{n} k \mid k, \dot{n}+\dot{1}}(\theta) \text { and } g_{k \dot{n} \mid \dot{n}, k+1}(\theta)
$$

have a simple pole at $\theta=0$.

Then, the commutativity of the left and right charges suggests that not all of the $2(N-1) N$ free fields are independent, and one should have the following relations

$$
\phi_{k, \dot{n}}+\dot{\phi}_{\dot{n}, k+1}=\phi_{k, \dot{n}+\dot{1}}+\dot{\phi}_{\dot{n}, k}, \quad k=1, \ldots, N-1, \dot{n}=\dot{1}, \ldots, \dot{N}-\dot{1} .
$$

Thus, it is expected that the number of independent fields is $N^{2}-1$ which matches the number of fields in the $S U(N)$ PCF Lagrangian. 
Notice that the zero modes $Q_{k \dot{r}}, Q_{\dot{k} r}, P_{k \dot{r}}$ and $P_{\dot{k} r}$ should satisfy

$$
Q_{k \dot{r}}=Q_{k}, \quad P_{k \dot{r}}=P_{k}, \quad Q_{\dot{k} r}=Q_{\dot{k}}, \quad P_{\dot{k} r}=P_{\dot{k}},
$$

where the zero modes $P_{k}, Q_{k}$ and $P_{\dot{k}}, Q_{\dot{k}}$ commute with the oscillators in the fields, and satisfy the algebra

$$
\left[P_{k}, Q_{n}\right]=i a_{k n}, \quad\left[Q_{k}, Q_{n}\right]=\left[P_{k}, P_{n}\right]=0, \quad k, n=0,1, \ldots, N .
$$

Here $a_{i j}=2 \delta_{i j}-\delta_{i-1, j}-\delta_{i+1, j}$ is the Cartan matrix of type $A_{N-1}$. Similar relations hold between $P_{\dot{k}}, Q_{\dot{k}}$. In addition the dotted and undotted zero modes commute.

\subsection{Ansatz for ZF operators and charges}

The ZF operators $\mathbb{A}_{k \dot{r}}^{\dagger}$ and the lowering operators $\mathbb{J}_{k}^{-}, \dot{\mathbb{J}}_{\dot{k}}^{-}$will be represented by the ZF vertex operators $Z_{k \dot{r}}$ and charges $\chi_{k}^{-}, \dot{\chi}_{\dot{k}}^{-}$. According to the discussion above, a rather general ansatz is

$$
\begin{aligned}
Z_{1 \dot{1}}(\theta) & =\rho(\theta) e^{i \phi_{0}(\theta)}, \\
\chi_{k}^{-} & =\sum_{\dot{r}=\dot{1}}^{\dot{N}} \int_{C_{k \dot{r}}} \frac{d \alpha_{k \dot{r}}}{2 \pi} c_{k \dot{r}}\left(\alpha_{k \dot{r}}\right) e^{i \phi_{k \dot{r}}\left(\alpha_{k \dot{r}}\right)}, \quad \dot{\chi}_{\dot{k}}^{-}=\sum_{r=1}^{N} \int_{C_{k r}} \frac{d \alpha_{\dot{k} r}}{2 \pi} c_{k r}\left(\alpha_{\dot{k}_{r}}\right) e^{i \dot{\phi}_{k r}\left(\alpha_{k r}\right)}, \\
Z_{k+1, \dot{r}}(\theta) & =\chi_{k}^{-} Z_{k \dot{r}}(\theta)-Z_{k \dot{r}}(\theta) \chi_{k}^{-}, \quad k=1, \ldots, N-1, \\
Z_{k, \dot{r}+1}(\theta) & =\dot{\chi}_{\dot{r}}^{-} Z_{k \dot{r}}(\theta)-Z_{k \dot{r}}(\theta) \dot{\chi}_{\dot{r}}^{-}, \quad \dot{r}=\dot{1}, \ldots, \dot{N}-\dot{1},
\end{aligned}
$$

where the functions $\rho(\theta), c_{k \dot{r}}(\alpha)$ and $c_{k r}(\alpha)$ satisfy some relations to be determined in the following sections. The free field $\phi_{0}$ can be expressed in terms of $\phi_{k \dot{r}}, \dot{\phi}_{k_{r}}$ due to the relations 2.15) of the extended ZF algebra, see (5.42). The integration contour $C$ in any operator $\chi$ which involves integration is fixed as follows [12]. The product of all vertex operators in a monomial containing $\chi$ is normal ordered. This produces a product of various Green's functions. The contour $C$ runs from $\operatorname{Re} \alpha=-\infty$ to $\operatorname{Re} \alpha=+\infty$ and it lies above all poles due to operators to the right of $\chi$ but below all poles due to operators to the left of $\chi$. The contour $C$ should be additionally deformed according to the procedure described if one then acts by the resulting monomial operator on other operators.

Recall, that

$$
V_{\mu}\left(\theta_{1}\right) V_{\nu}\left(\theta_{2}\right)=g_{\nu \mu}\left(\theta_{2}-\theta_{1}\right): V_{\mu}\left(\theta_{1}\right) V_{\nu}\left(\theta_{2}\right):,
$$

and $\mu, \nu$ can be 0 , or $k \dot{r}$ or $\dot{k}$. Thus, one has the following Green's functions

$$
g_{00}=g_{P C F}, \quad g_{0 \mid k \dot{r}}, g_{k \dot{r} \mid 0}, \quad g_{0 \mid k r}, g_{\dot{k} \mid 0}, \quad g_{k \dot{r} \mid n \dot{q}}, \quad g_{\dot{k} r \mid \dot{n} q}, \quad g_{k \dot{r} \mid \dot{n} q}, g_{\dot{n} q \mid k \dot{r}},
$$

and the corresponding S-matrices. The Green's functions are listed in subsection 3.3, and their derivation is sketched in appendix A. In what follows $\phi_{k \dot{r}}$ and $\phi_{k r}$ are sometimes referred to as the left and right sector fields, respectively. Their Green's functions 
$g_{k \dot{r} \mid n \dot{q}}, g_{\dot{k} \mid \dot{n} q}, g_{k \dot{r} \mid \dot{n} q}, g_{\dot{n} q \mid k \dot{r}}$ are referred to as the left-left, right-right, left-right, and rightleft functions.

By using these formulae, one finds in particular

$$
\begin{aligned}
& Z_{2 \mathrm{i}}(\theta)=\chi_{1}^{-} Z_{1 \dot{1}}(\theta)-Z_{1 \mathrm{i}}(\theta) \chi_{1}^{-}=\rho(\theta) \sum_{\dot{r}} \int_{C_{1 \dot{r}}} \frac{d \alpha_{1 \dot{r}}}{2 \pi} c_{1 \dot{r}}\left(\alpha_{1 \dot{r}}\right) g_{0 \mid \dot{1}}^{a}\left(\theta-\alpha_{1 \dot{r}}\right) e^{i \phi_{0}(\theta)+i \phi_{1 \dot{r}}\left(\alpha_{1 \dot{r}}\right)}, \\
& Z_{1 \dot{2}}(\theta)=\dot{\chi}_{\dot{1}}^{-} Z_{1 \dot{1}}(\theta)-Z_{1 \dot{1}}(\theta) \dot{\chi}_{\dot{1}}^{-}=\rho(\theta) \sum_{r} \int_{C_{\dot{1} r}} \frac{d \alpha_{\dot{1} r}}{2 \pi} c_{\dot{1}_{r}}\left(\alpha_{\dot{1}_{r}}\right) g_{0 \mid \dot{1} r}^{a}\left(\theta-\alpha_{\dot{1}_{r}}\right) e^{i \phi_{0}(\theta)+i \dot{\phi}_{\dot{1}}\left(\alpha_{\dot{1}}\right)},
\end{aligned}
$$

where the integration contours run above the poles of $g_{0 \mid A}$ functions, and below the poles of $g_{A \mid 0}$ functions, and for any Green's function one defines

$$
g_{A \mid B}^{a}(\alpha-\beta) \equiv g_{A \mid B}(\alpha-\beta)-g_{B \mid A}(\beta-\alpha) .
$$

To simplify formulae the summation symbols and the differentials $\frac{d \alpha_{k \dot{r}}}{2 \pi}, \frac{d \alpha_{k r}}{2 \pi}$, and the dependance of free fields and Green's functions on their arguments are often dropped: $\phi_{A} \equiv \phi_{A}\left(\alpha_{A}\right), g_{A \mid B} \equiv g_{A \mid B}\left(\alpha_{A}-\alpha_{B}\right)$, e.g.

$$
\begin{aligned}
& \phi_{0} \equiv \phi_{0}(\theta), \quad \phi_{k \dot{r}} \equiv \phi_{k \dot{r}}\left(\alpha_{k \dot{r}}\right), \quad \dot{\phi}_{k r} \equiv \dot{\phi}_{\dot{k} r}\left(\alpha_{\dot{k}_{r}}\right) \\
& g_{0 \mid 1 \dot{r}} \equiv g_{0 \mid 1 \dot{r}}\left(\theta-\alpha_{\dot{r}}\right), \quad g_{\dot{k} \mid n \dot{r}} \equiv g_{\dot{k} q \mid n \dot{r}}\left(\alpha_{\dot{k} q}-\alpha_{n \dot{r}}\right),
\end{aligned}
$$

unless there is an ambiguity.

The ZF operator $Z_{m \dot{n}}$ then can be symbolically written as follows

$$
\begin{aligned}
Z_{m \dot{n}}(\theta) & =\rho(\theta) \int \prod_{k=1}^{m-1} c_{k \dot{r}_{k}} g_{k-1, \dot{r}_{k-1} \mid k \dot{r}_{k}}^{a} \prod_{\dot{k}=\dot{1}}^{\dot{n}-1} c_{k r_{k}} g_{\dot{k}-\dot{1}, r_{\dot{k}-1} \mid k_{k}}^{a} \prod_{k=1}^{m-1} \prod_{\dot{k}=\dot{1}}^{\dot{n}-1} g_{\dot{k} r_{k} \mid k \dot{r}_{k}}^{a} \\
& \times \exp \left(i \phi_{0}+i \sum_{k=1}^{m-1} \phi_{k \dot{r}_{k}}+i \sum_{\dot{k}=\dot{1}}^{\dot{n}-\dot{1}} \dot{\phi}_{\dot{k}_{r_{k}}}\right)
\end{aligned}
$$

where $g_{0 \dot{r}_{0} \mid 1 \dot{r}_{1}}^{a} \equiv g_{0 \mid 1 \dot{r}_{1}}^{a}$, the sum over $\dot{r}_{k}$ and $r_{\dot{k}}$ is taken, and in each term of the sum the integration contours run according to the rule decribed above.

\subsection{List of Green's functions}

Here are all Green's functions different from 1 collected.

$$
\begin{gathered}
g_{0 \mid 1 \mathrm{i}}(\theta)=g_{0 \mid \mathrm{i} 1}(\theta)=\frac{\theta-\frac{2 \pi i}{N}}{\theta}, \quad g_{1 \dot{\mathrm{i} \mid 0}}(\theta)=g_{\mathrm{i} 1 \mid 0}(\theta)=1, \\
g_{0 \mid \dot{r}}(\theta)=g_{0 \mid \dot{\mathrm{i}} r}(\theta)=1, \quad g_{1 \dot{r} \mid 0}(\theta)=g_{\mathrm{i} r \mid 0}(\theta)=\frac{\theta}{\theta+\frac{2 \pi i}{N}}, \quad \dot{1}<\dot{r}, 1<r, \\
g_{k \dot{r} \mid k \dot{q}}(\theta)=g_{\dot{k}_{r \mid k q}}(\theta)=1, \quad g_{k \dot{q} \mid k \dot{r}}(\theta)=g_{\dot{k} q \mid k r}(\theta)=\frac{\theta+\frac{2 \pi i}{N}}{\theta-\frac{2 \pi i}{N}}, \quad \dot{r}<\dot{q}, r<q,
\end{gathered}
$$




$$
\begin{gathered}
g_{k \dot{r} \mid k \dot{r}}(\theta)=g_{\dot{k} r \mid k r}(\theta)=\frac{\theta}{\theta-\frac{2 \pi i}{N}}, \quad \dot{k}, \dot{r} \geq \dot{1}, k, r \geq 1, \\
g_{k \dot{r} \mid k+1, \dot{q}}(\theta)=g_{\dot{k} \mid \dot{k}+\dot{1}, q}(\theta)=\frac{\theta-\frac{2 \pi i}{N}}{\theta}, \quad g_{k+1, \dot{q} \mid k \dot{r}}(\theta)=g_{\dot{k}+\dot{1}, q \mid \dot{k} r}(\theta)=1, \quad \dot{r} \geq \dot{q}, r \geq q, \\
g_{k \dot{r} \mid k+1, \dot{q}}(\theta)=g_{k r \mid \dot{k}+\dot{1}, q}(\theta)=1, \quad g_{k+1, \dot{q} \mid k, \dot{r}}(\theta)=g_{\dot{k}+\dot{1}, q \mid \dot{k} r}(\theta)=\frac{\theta}{\theta+\frac{2 \pi i}{N}}, \quad \dot{r}<\dot{q}, r<q, \\
g_{k \dot{r} \mid \dot{r}, k+1}(\theta)=g_{\dot{r} k \mid k, \dot{r}+\dot{1}}(\theta)=\frac{\theta-\frac{2 \pi i}{N}}{\theta}, \quad g_{\dot{r}, k+1 \mid k \dot{r}}(\theta)=g_{k, \dot{r}+\dot{1} \mid \dot{r} k}(\theta)=\frac{\theta+\frac{2 \pi i}{N}}{\theta} .
\end{gathered}
$$

The Green's functions satisfy the following equations

$$
g_{A \mid k \dot{r}}(\theta) g_{A \mid \dot{r}, k+1}(\theta)=g_{A \mid \dot{r} k}(\theta) g_{A \mid k, \dot{r}+\dot{1}}(\theta), \quad g_{k \dot{r} \mid A}(\theta) g_{\dot{r}, k+1 \mid A}(\theta)=g_{\dot{r} k \mid A}(\theta) g_{k, \dot{r}+\dot{1} \mid A}(\theta),
$$

where $A$ is any of the indices of the fields. These equations are obtained from the following constraints between the free fields

$$
\phi_{k \dot{r}}(\theta)+\dot{\phi}_{\dot{r}, k+1}(\theta)=\dot{\phi}_{\dot{r} k}(\theta)+\phi_{k, \dot{r}+\dot{1}}(\theta) .
$$

Then, the functions $c_{k \dot{r}}(\alpha)$ and $c_{k r}(\alpha)$ satisfy the equations

$$
c_{k \dot{r}}(\theta) c_{\dot{r}, k+1}(\theta)=c_{\dot{r} k}(\theta) c_{k, \dot{r}+\dot{1}}(\theta) .
$$

Both (3.31) and 3.32 are derived from the commutativity of the left and right algebras charges.

The constraints (3.31) are solved in the appendix B, and the fields $\phi_{k \dot{r}}, \dot{\phi}_{\dot{r} k}$ and $\phi_{0}$ are expressed in terms of the $N^{2}-1$ elementary free fields defined in (B.31).

It is worthwhile to mention that shifting the variables $\alpha_{k \dot{r}}$ as

$$
\alpha_{k \dot{r}} \rightarrow \alpha_{k \dot{r}}-\frac{\pi i}{N} k, \quad \alpha_{k r} \rightarrow \alpha_{k r}-\frac{\pi i}{N} \dot{k},
$$

one can transform all left-left and right-right functions to the ones which have zeroes and poles at the same locations as the Gross-Neveu functions. However, the left-right Green's functions would have zeroes and poles at positions which depend on their indices. To be precise

$$
\begin{aligned}
& g_{k \dot{r} \mid \dot{r}, k+1}(\theta) \rightarrow \frac{\theta-\frac{\pi i}{N}(2+k-\dot{r})}{\theta-\frac{\pi i}{N}(k-\dot{r})}, \quad g_{\dot{r}, k+1 \mid k \dot{r}}(\theta) \rightarrow \frac{\theta+\frac{\pi i}{N}(2+k-\dot{r})}{\theta+\frac{\pi i}{N}(k-\dot{r})}, \\
& g_{\dot{r} k \mid k, \dot{r}+\dot{1}}(\theta) \rightarrow \frac{\theta-\frac{\pi i}{N}(2+\dot{r}-k)}{\theta-\frac{\pi i}{N}(\dot{r}-k)}, \quad g_{k, \dot{r}+\dot{1} \mid \dot{r} k}(\theta) \rightarrow \frac{\theta+\frac{\pi i}{N}(2+\dot{r}-k)}{\theta+\frac{\pi i}{N}(\dot{r}-k)} .
\end{aligned}
$$




\subsection{The angular Hamiltonian}

An important step in constructing a free field representation is to find an angular Hamiltonian. The most general Hamiltonian is of the form

$$
\mathbb{K}=i \int_{0}^{\infty} d t \sum_{A, B} h_{B A}(t) a_{A}(-t) a_{B}(t)
$$

where the sum runs over all independent elementary operators. The relations to be satisfied are

$$
\frac{d}{d \theta} Z_{m \dot{n}}(\theta)=-\left[\mathbb{K}, Z_{m \dot{n}}(\theta)\right]-i \Omega_{m \dot{n}} Z_{m \dot{n}}(\theta) .
$$

Computing the derivative of (3.24) with respect to $\theta$ it is straightforward to show that if

$$
\rho(\theta)=e^{i \varkappa \theta} \tilde{\rho}, \quad c_{k \dot{r}}(\theta)=e^{i \varkappa_{k} \theta} \tilde{c}_{k \dot{r}}, \quad c_{k r}(\theta)=e^{i \varkappa_{k} \theta} \tilde{c}_{k_{r}},
$$

then

$$
\begin{aligned}
\frac{d}{d \theta} Z_{m \dot{n}}(\theta) & =-i \Omega_{m \dot{n}} Z_{m \dot{n}}(\theta) \\
& +\rho(\theta) \int \prod_{k=1}^{m-1} c_{k \dot{r}_{k}} g_{k-1, \dot{r}_{k-1} \mid k \dot{r}_{k}}^{a} \prod_{\dot{k}=\dot{1}}^{\dot{n}-\dot{1}} c_{k r_{k}} g_{\dot{k}-\dot{1}, r_{\dot{k}-1} \mid}^{a} \mid \dot{k}_{\dot{k}} \prod_{k=1}^{m-1} \prod_{\dot{k}=\dot{1}}^{\dot{n}-\dot{1}} g_{\dot{k} r_{\dot{k}} \mid k \dot{r}_{k}}^{a} \\
& \times\left(\frac{\partial}{\partial \theta}+\sum_{k=1}^{m-1} \frac{\partial}{\partial \alpha_{k \dot{r}_{k}}}+\sum_{\dot{k}=\dot{1}}^{\dot{n}-\dot{1}} \frac{\partial}{\partial \alpha_{\dot{k} r_{k}}}\right) \exp \left(i \phi_{0}+i \sum_{k=1}^{m-1} \phi_{k \dot{r}_{k}}+i \sum_{\dot{k}=\dot{1}}^{\dot{n}-\dot{1}} \phi_{\dot{k} r_{\dot{k}}}\right),
\end{aligned}
$$

where $\varkappa, \varkappa_{k}, \varkappa_{\dot{k}}, \tilde{\rho}, \tilde{c}_{k \dot{r}}, \tilde{c}_{k r}$ are constants, and

$$
\Omega_{m \dot{n}}=-\left(\varkappa+\sum_{k=1}^{m-1} \varkappa_{k}+\sum_{\dot{k}=\dot{1}}^{\dot{n}-\dot{1}} \varkappa_{\dot{k}}\right) .
$$

Thus it is sufficient to find $\mathbb{K}$ such that

$$
\left[\mathbb{K}, V_{\mu}(\theta)\right]=-\frac{d}{d \theta} V_{\mu}(\theta)
$$

where $V_{\mu}$ is any vertex operator

$$
V_{\mu}(\theta)=e^{i \phi_{\mu}(\theta)}, \quad \phi_{\mu}(\theta)=\int_{-\infty}^{\infty} \frac{d t}{i t} \Phi_{\mu A}(t) a_{A}(t) e^{i \theta t}
$$

where the index $A$ runs over the indices of the $N^{2}-1$ independent elementary operators. One gets

$$
-\frac{d}{d \theta} V_{\mu}(\theta)=-i \int_{-\infty}^{\infty} d t e^{i \theta t}: \Phi_{\mu A}(t) a_{A}(t) e^{i \phi_{\mu}(\theta)}:
$$


and

$$
\begin{aligned}
{\left[\mathbb{K}, V_{\mu}(\theta)\right]=:\left[\mathbb{K}, i \phi_{\mu}(\theta)\right] e^{i \phi_{\mu}(\theta)}: } & =-i \int_{-\infty}^{0} d t e^{i \theta t} \Phi_{\mu B}(t) h_{B A}(-t): a_{A}(t) e^{i \phi_{\mu}(\theta)}: \\
& -i \int_{0}^{\infty} d t e^{i \theta t} \Phi_{\mu B}(t) h_{A B}(t): a_{A}(t) e^{i \phi_{\mu}(\theta)}:
\end{aligned}
$$

where it is used that $f_{A B}(t)=\delta_{A B}$. Thus, $h_{A B}(t)=\delta_{A B}$, and the angular Hamiltonian is given by the following simple formula

$$
\mathbb{K}=i \int_{0}^{\infty} d t \sum_{A} a_{A}(-t) a_{A}(t)
$$

\subsection{Commutativity relations}

The charges and ZF operators must satisfy the commutativity relations

$$
\begin{gathered}
{\left[\chi_{k}^{-}, \chi_{n}^{-}\right]=0, \quad|k-n| \neq 1,} \\
{\left[\left[\chi_{\dot{k}}^{-}, \chi_{\bar{k}+1}^{-}\right], \chi_{\dot{k}}^{-}\right]=0, \quad\left[\left[\chi_{k}^{-}, \chi_{k+1}^{-}\right], \chi_{k+1}^{-}\right]=0,} \\
{\left[\dot{\chi}_{\dot{k}}^{-}, \dot{\chi}_{\dot{n}}^{-}\right]=0, \quad|\dot{k}-\dot{n}| \neq \dot{1},} \\
{\left[\left[\dot{\chi}_{\dot{k}}^{-}, \dot{\chi}_{\dot{k}+\dot{1}}^{-}\right], \dot{\chi}_{\dot{k}}^{-}\right]=0, \quad\left[\left[\dot{\chi}_{\dot{k}}^{-}, \dot{\chi}_{\dot{k}+\dot{1}}^{-}\right], \dot{\chi}_{\dot{k}+\dot{1}}^{-}\right]=0,} \\
{\left[\chi_{\dot{k}}^{-}, \dot{\chi}_{\dot{n}}^{-}\right]=0, \quad \forall k, \dot{n}, \quad \text { unless } \quad m=k,} \\
\chi_{\dot{k}}^{-} Z_{m \dot{n}}(\theta)=Z_{m \dot{n}}(\theta) \chi_{k}^{-}, \quad \dot{1} \dot{n} \leq \dot{N}, \quad \text { unless } \quad \dot{m}=\dot{k}, \\
\dot{\chi}_{\dot{k}}^{-} Z_{n \dot{m}}(\theta)=Z_{n \dot{m}}(\theta) \dot{\chi}_{\dot{k}}^{-}, \quad 1 \leq n \leq N, \quad
\end{gathered}
$$

which follow from the $\mathfrak{s u}(N) \oplus \mathfrak{s u}(N)$ symmetry algebra of the model.

The relations 3.46) and 3.48 just imply that the Green's functions $g_{k \dot{r} \mid l \dot{q}}(\theta)=$ $g_{k_{r} \mid i_{q}}(-\theta)$ have no poles unless $|k-l|=1,|\dot{k}-\dot{l}|=\dot{1}$. The simplest choice used in this paper is $g_{k \dot{r} \mid l \dot{q}}=g_{k_{r} \mid \dot{i}_{q}}=1$. Then, the commutativity 3.50 of the left and right charges leads to the relations (3.31) and (3.32), and together with (3.46) and (3.48) guarantees that (3.51) and (3.52) hold as soon as the relations

$$
\begin{array}{ll}
\chi_{k}^{-} Z_{k+1, \mathrm{i}}(\theta)=Z_{k+1, \mathrm{i}}(\theta) \chi_{k}^{-}, & \chi_{k}^{-} Z_{k+2, \mathrm{i}}(\theta)=Z_{k+2, \mathrm{i}}(\theta) \chi_{k}^{-}, \\
\dot{\chi}_{\dot{k}}^{-} Z_{1, \dot{k}+\mathrm{i}}(\theta)=Z_{1, \dot{k}+\mathrm{i}}(\theta) \dot{\chi}_{\dot{k}}^{-}, & \dot{\chi}_{\dot{k}}^{-} Z_{1, \dot{k}+\dot{2}}(\theta)=Z_{1, \dot{k}+\dot{2}}(\theta) \dot{\chi}_{\dot{k}}^{-},
\end{array}
$$

are satisfied. The relations (3.53) for $k>1$ follow from $(3.47)$ and $(3.49)$ which can be easily verified. The commutativity of $\chi_{1}^{-}$with $Z_{2 \dot{1}}$, and $\dot{\chi}_{\dot{1}}^{-}$with $Z_{1 \dot{2}}$ is proven in next section. 


\section{The ZF algebra}

\subsection{The ZF relations}

The ZF algebra relations for $Z_{k \dot{r}}, Z_{l \dot{q}}$ take the form

$$
\begin{aligned}
Z_{k \dot{r}}\left(\theta_{1}\right) Z_{l \dot{q}}\left(\theta_{2}\right)=S\left(\theta_{12}\right)[ & s_{12}^{2} Z_{k \dot{r}}\left(\theta_{2}\right) Z_{l \dot{q}}\left(\theta_{1}\right)+s_{21}^{2} Z_{l \dot{q}}\left(\theta_{2}\right) Z_{k \dot{r}}\left(\theta_{1}\right) \\
& \left.+s_{12} s_{21}\left(Z_{k \dot{q}}\left(\theta_{2}\right) Z_{l \dot{r}}\left(\theta_{1}\right)+Z_{l \dot{r}}\left(\theta_{2}\right) Z_{k \dot{q}}\left(\theta_{1}\right)\right)\right]
\end{aligned}
$$

where

$$
s_{12}=-\frac{\frac{2 \pi i}{N}}{\theta_{12}-\frac{2 \pi i}{N}}, \quad s_{21}=\frac{\theta_{12}}{\theta_{12}-\frac{2 \pi i}{N}}, \quad s_{12}+s_{21}=1 .
$$

The relations simplify for $\dot{q}=\dot{r}$

$$
Z_{k \dot{r}}\left(\theta_{1}\right) Z_{l \dot{r}}\left(\theta_{2}\right)=S\left(\theta_{12}\right)\left[s_{12} Z_{k \dot{r}}\left(\theta_{2}\right) Z_{l \dot{r}}\left(\theta_{1}\right)+s_{21} Z_{l \dot{r}}\left(\theta_{2}\right) Z_{k \dot{r}}\left(\theta_{1}\right)\right]
$$

and for $l=k$

$$
Z_{k \dot{r}}\left(\theta_{1}\right) Z_{k \dot{q}}\left(\theta_{2}\right)=S\left(\theta_{12}\right)\left[s_{12} Z_{k \dot{r}}\left(\theta_{2}\right) Z_{k \dot{q}}\left(\theta_{1}\right)+s_{21} Z_{k \dot{q}}\left(\theta_{2}\right) Z_{k \dot{r}}\left(\theta_{1}\right)\right]
$$

and they take the simplest form for $l=k, \dot{q}=\dot{r}$

$$
Z_{k \dot{r}}\left(\theta_{1}\right) Z_{k \dot{r}}\left(\theta_{2}\right)=S\left(\theta_{12}\right) Z_{k \dot{r}}\left(\theta_{2}\right) Z_{k \dot{r}}\left(\theta_{1}\right)
$$

Up to the S-matrix $S\left(\theta_{12}\right)$, the relations (4.3) or (4.4) are the same as the ZF algebra relations of the Gross-Neveu model.

All these ZF relations follow from the commutativity relations discussed in subsection 3.5. and the ZF algebra relations for $Z_{11}, Z_{2 \dot{1}}, Z_{1 \dot{2}}, Z_{2 \dot{2}}$. Indeed, assume that the $\mathrm{ZF}$ relations (4.1) have been proven for all indices which are less or equal to $k, l, \dot{r}, \dot{q}$. It is then easy to prove that the ZF relations hold for $k+1, l, \dot{r}, \dot{q}$. Indeed, for $l \neq k$ one gets

$$
\begin{aligned}
Z_{k+1, \dot{r}}\left(\theta_{1}\right) Z_{l \dot{q}}\left(\theta_{2}\right)= & \left(\chi_{k}^{-} Z_{k \dot{r}}\left(\theta_{1}\right)-Z_{k \dot{r}}\left(\theta_{1}\right) \chi_{k}^{-}\right) Z_{l \dot{q}}\left(\theta_{2}\right) \\
= & \chi_{k}^{-} Z_{k \dot{r}}\left(\theta_{1}\right) Z_{l \dot{q}}\left(\theta_{2}\right)-Z_{k \dot{r}}\left(\theta_{1}\right) Z_{l \dot{q}}\left(\theta_{2}\right) \chi_{k}^{-} \\
= & S\left(\theta_{12}\right)\left[s_{12}^{2} Z_{k+1, \dot{r}}\left(\theta_{2}\right) Z_{l \dot{q}}\left(\theta_{1}\right)+s_{21}^{2} Z_{l \dot{q}}\left(\theta_{2}\right) Z_{k+1, \dot{r}}\left(\theta_{1}\right)\right. \\
& \left.\quad+s_{12} s_{21}\left(Z_{k+1, \dot{q}}\left(\theta_{2}\right) Z_{l \dot{r}}\left(\theta_{1}\right)+Z_{l \dot{r}}\left(\theta_{2}\right) Z_{k+1, \dot{q}}\left(\theta_{1}\right)\right)\right],
\end{aligned}
$$


while if $l=k \geq 2$ then

$$
\begin{aligned}
Z_{k+1, \dot{r}}\left(\theta_{1}\right) Z_{k \dot{q}}\left(\theta_{2}\right)= & Z_{k+1, \dot{r}}\left(\theta_{1}\right)\left(\chi_{k-1}^{-} Z_{l-1, \dot{q}}\left(\theta_{2}\right)-Z_{k-1, \dot{q}}\left(\theta_{2}\right) \chi_{k-1}^{-}\right) \\
= & \chi_{k-1}^{-} Z_{k+1, \dot{r}}\left(\theta_{1}\right) Z_{k-1, \dot{q}}\left(\theta_{2}\right)-Z_{k+1, \dot{r}}\left(\theta_{1}\right) Z_{k-1, \dot{q}}\left(\theta_{2}\right) \chi_{\bar{k}-1}^{-} \\
= & {\left[\chi_{k-1}^{-}, S\left(\theta_{12}\right)\left[s_{12}^{2} Z_{k+1, \dot{r}}\left(\theta_{2}\right) Z_{k-1, \dot{q}}\left(\theta_{1}\right)+s_{21}^{2} Z_{k-1, \dot{q}}\left(\theta_{2}\right) Z_{k+1, \dot{r}}\left(\theta_{1}\right)\right.\right.} \\
& \left.\left.\quad+s_{12} s_{21}\left(Z_{k+1, \dot{q}}\left(\theta_{2}\right) Z_{k-1, \dot{r}}\left(\theta_{1}\right)+Z_{k-1, \dot{r}}\left(\theta_{2}\right) Z_{k+1, \dot{q}}\left(\theta_{1}\right)\right)\right]\right] \\
= & S\left(\theta_{12}\right)\left[s_{12}^{2} Z_{k+1, \dot{r}}\left(\theta_{2}\right) Z_{k \dot{q}}\left(\theta_{1}\right)+s_{21}^{2} Z_{k \dot{q}}\left(\theta_{2}\right) Z_{k+1, \dot{r}}\left(\theta_{1}\right)\right. \\
& \left.\quad+s_{12} s_{21}\left(Z_{k+1, \dot{q}}\left(\theta_{2}\right) Z_{k \dot{r}}\left(\theta_{1}\right)+Z_{k \dot{r}}\left(\theta_{2}\right) Z_{k+1, \dot{q}}\left(\theta_{1}\right)\right)\right] .
\end{aligned}
$$

Obviously, the consideration of the other indices follows the same line. Thus, it is sufficient to prove that $Z_{1 \dot{1}}, Z_{21}, Z_{1 \dot{2}}, Z_{2 \dot{2}}$ satisfy the ZF algebra.

\subsection{Integration contours and the $\mathrm{ZF}$ operators $Z_{2 \dot{1}}, Z_{1 \dot{2}}, Z_{2 \dot{2}}$}

To simplify the computations it is often convenient to choose a particular ordering of integration contours in $Z_{m \dot{n}}(\theta)$ operators. In what follows in each $Z_{m \dot{n}}(\theta)$ all the integration contours $C_{k \dot{r}}$ and $C_{\dot{k} r}$ are shifted below $\theta^{--} \equiv \theta-2 \pi i / N$. Then, the contours are arranged as follows

$$
\begin{array}{ccc}
Z_{m \dot{n}}(\theta): & \theta^{--} / C_{1 \dot{r}} / C_{\dot{1} r} / C_{2 \dot{q}} / C_{\dot{2} q} / \cdots / C_{m-1, \dot{s}} / C_{\dot{m}-\dot{1}, s} / \cdots / C_{\dot{n}-\dot{1}, w}, \quad m \leq \dot{n}, \\
Z_{m \dot{n}}(\theta): & \theta^{--} / C_{1 \dot{r}} / C_{\dot{1} r} / C_{2 \dot{q}} / C_{\dot{2} q} / \cdots / C_{n-1, \dot{s}} / C_{\dot{n}-\dot{1}, s} / \cdots / C_{m-1, \dot{w}}, \quad m>\dot{n},
\end{array}
$$

where $\dot{n}$ and $n$ are identified, and the notation $C_{1 \dot{r}} / C_{\dot{1} r}$ means that the contour $C_{1 \dot{r}}$ is a bit above $C_{\dot{1} r}$. Then, the contours $C_{k \dot{r}}$ and $C_{k+1, \dot{r}}$ are separated by $2 \pi i / N$. Finally, for all $k, \dot{k}, \dot{r}, \dot{q}, r, q$ one takes $C_{k \dot{r}}=C_{k \dot{q}}$ and $C_{k_{r}}=C_{\dot{k} q}$. This choice makes convenient to use the specific path to any ZF operator, see (4.9).

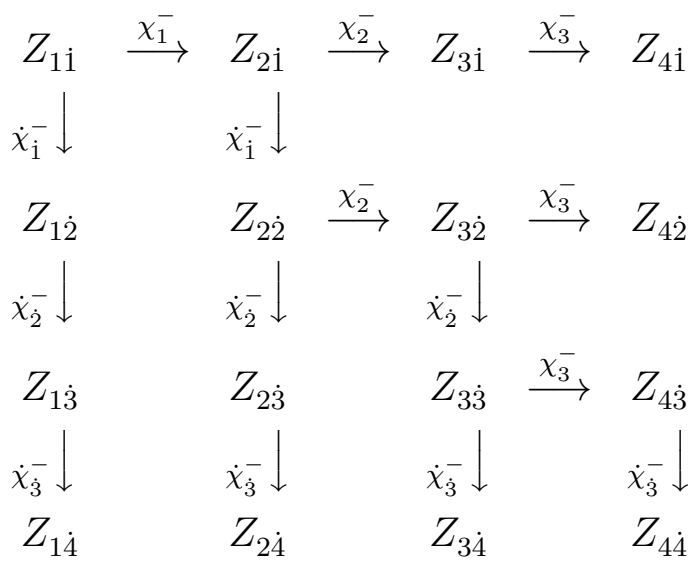

It is also particularly useful to analyse the highest weight $r$-particle bound state vertex operators discussed in section 5 . 
All integration contours in the formulae below are canonically ordered, and the notation $R_{A \mid B} \equiv i r_{A \mid B}$ is used where $r_{A \mid B}$ is the residue of $g_{A \mid B}$. Since any of the Green's functions has no more than one pole there is no ambiguity in the definition.

The derivation of the ZF operators $Z_{21}, Z_{1 \dot{2}}, Z_{2 \dot{2}}$ is given in appendix C.

One gets $Z_{21}$ and $Z_{1 \dot{2}}$

$$
\begin{aligned}
Z_{2 \mathrm{i}}(\theta) & =Z_{2 \dot{1}}^{(1)}(\theta)+Z_{2 \dot{1}}^{(2)}(\theta) \\
& =\rho(\theta) \int_{\theta^{--} / C_{1 \dot{r}}} c_{1 \dot{r}} g_{0 \mid \dot{1}}^{a} e^{i \phi_{0}+i \phi_{1 \dot{r}}}+\rho(\theta) c_{1 \dot{\mathrm{i}}}(\theta) R_{0 \mid 1 \dot{1}} e^{i \phi_{0}+i \phi_{1 \dot{1}}(\theta)}, \\
Z_{1 \dot{2}}(\theta) & =Z_{1 \dot{2}}^{(1)}(\theta)+Z_{1 \dot{2}}^{(2)}(\theta) \\
& =\rho(\theta) \int_{\theta^{--} / C_{\mathrm{i} r}} c_{\dot{1} r} g_{0 \mid \dot{1} r}^{a} e^{i \phi_{0}+i \phi_{\mathrm{i} r}}+\rho(\theta) c_{\dot{1} 1}(\theta) R_{0 \mid \dot{1} 1} e^{i \phi_{0}(\theta)+i \dot{\phi}_{\mathrm{i} 1}(\theta)} .
\end{aligned}
$$

Note that there is no pole at $\theta_{12}=\mathfrak{u}_{2}$ in the products $Z_{1 \mathrm{i}}\left(\theta_{1}\right) Z_{2 \mathrm{i}}\left(\theta_{2}\right)$ and $Z_{1 \mathrm{i}}\left(\theta_{1}\right) Z_{1 \dot{2}}\left(\theta_{2}\right)$ because, e.g. $R_{0 \mid \mathrm{i} r}=\delta_{r 1} R_{0 \mid \mathrm{i} 1}$ but $g_{1 \mathrm{i} \mid 0}=1$.

The operator $Z_{22}$ is given by

$$
\begin{aligned}
& Z_{2 \dot{2}}(\theta)=\sum_{a=1}^{6} Z_{2 \dot{2}}^{(a)}(\theta) \\
& Z_{2 \dot{2}}^{(1)}(\theta)=\rho(\theta) \int_{\theta^{--} / C_{1 \dot{r}} / C_{\dot{1} q}} c_{1 \dot{r}} c_{\mathrm{1}_{q}} g_{0 \mid 1 \dot{r}}^{a} g_{0 \mid \dot{1} q}^{a} g_{\dot{\mathrm{i}} q \mid 1 \dot{r}} e^{i \phi_{0}+i \phi_{1 \dot{r}}+i \dot{\phi}_{\mathrm{i} q}}, \\
& Z_{2 \dot{2}}^{(2)}(\theta)=\rho(\theta) \int_{\theta^{--} / C_{\mathrm{i}_{q}}} c_{1 \mathrm{i}}(\theta) c_{\mathrm{i} q} R_{0 \mid 1 \mathrm{i}} g_{0 \mid \mathrm{i} q}^{a} g_{\mathrm{i} q \mid 1 \mathrm{i}}\left(\alpha_{\dot{1}_{q}}-\theta\right) e^{i \phi_{0}+i \phi_{1 \mathrm{i}}(\theta)+i \dot{\phi}_{\mathrm{i}_{q}}}, \\
& Z_{2 \dot{2}}^{(3)}(\theta)=\rho(\theta) \int_{\theta^{--} / C_{1 \dot{r}}} c_{1 \dot{r}} c_{\dot{1} 1}(\theta) g_{0 \mid 1 \dot{r}}^{a} R_{0 \mid \dot{1} 1} g_{1 \dot{r} \mid \dot{1} 1}\left(\alpha_{1 \dot{r}}-\theta\right) e^{i \phi_{0}+i \phi_{1 \dot{r}}+i \dot{\phi}_{11}(\theta)}, \\
& Z_{2 \dot{2}}^{(4)}(\theta)=\rho(\theta) \int_{\theta^{--} / C_{1 \dot{r}}} c_{1 \dot{r}} c_{\dot{1} q}\left(\alpha_{1 \dot{r}}\right) g_{0 \mid 1 \dot{r}}^{a} g_{0 \mid \dot{1} q}\left(\theta-\alpha_{1 \dot{r}}\right) R_{1 \dot{r} \mid \dot{1} q} e^{i \phi_{0}+i \phi_{1 \dot{r}}+i \dot{\phi}_{1 q}\left(\alpha_{1 \dot{r}}\right)}, \\
& Z_{2 \dot{2}}^{(5)}(\theta)=\rho(\theta) c_{1 \mathrm{i}}(\theta) c_{\mathrm{i} 1}(\theta) R_{0 \mid 1 \mathrm{i}} R_{0 \mid \mathrm{i} 1} e^{i \phi_{0}+i \phi_{1 \mathrm{i}}(\theta)+i \dot{\phi}_{\mathrm{i} 1}(\theta)}, \\
& Z_{2 \dot{2}}^{(6)}(\theta)=\rho(\theta) c_{1 \mathrm{i}}(\theta) c_{\mathrm{i} 2}(\theta) R_{0 \mid 1 \mathrm{i}} R_{1 \mathrm{i} \mid \mathrm{i} 2} e^{i \phi_{0}+i \phi_{1 \mathrm{i}}(\theta)+i \dot{\phi}_{\mathrm{i} 2}(\theta)} .
\end{aligned}
$$

\subsection{The $\mathrm{ZF}$ algebra for $Z_{1 \dot{1}}, Z_{2 \dot{1}}, Z_{1 \dot{2}}, Z_{2 \dot{2}}$}

It is not difficult to show that all ZF relations for $Z_{1 \dot{1}}, Z_{2 \dot{1}}, Z_{1 \dot{2}}, Z_{2 \dot{2}}$ follow from the commutativity of the left and right charges

$$
\chi_{1}^{-} \dot{\chi}_{\dot{1}}^{-}=\dot{\chi}_{\dot{1}}^{-} \chi_{1}^{-}
$$

the commutativity of the charges with the following ZF operators

$$
\chi_{1}^{-} Z_{2 \dot{k}}(\theta)=Z_{2 \dot{k}}(\theta) \chi_{1}^{-}, \quad \dot{\chi}_{\dot{1}}^{-} Z_{k \dot{2}}(\theta)=Z_{k \dot{2}}(\theta) \dot{\chi}_{\dot{1}}^{-}, \quad \dot{k}=\dot{1}, \dot{2}, \quad k=1,2,
$$


and the basic relations

$$
\begin{gathered}
Z_{1 \mathrm{i}}\left(\theta_{1}\right) Z_{1 \mathrm{i}}\left(\theta_{2}\right)=S\left(\theta_{12}\right) Z_{1 \mathrm{i}}\left(\theta_{2}\right) Z_{1 \mathrm{i}}\left(\theta_{1}\right) \\
Z_{1 \mathrm{i}}\left(\theta_{1}\right) Z_{2 \mathrm{i}}\left(\theta_{2}\right)=S\left(\theta_{12}\right)\left[s_{12} Z_{1 \mathrm{i}}\left(\theta_{2}\right) Z_{2 \mathrm{i}}\left(\theta_{1}\right)+s_{21} Z_{2 \mathrm{i}}\left(\theta_{2}\right) Z_{1 \mathrm{i}}\left(\theta_{1}\right)\right] \\
Z_{1 \mathrm{i}}\left(\theta_{1}\right) Z_{1 \dot{2}}\left(\theta_{2}\right)=S\left(\theta_{12}\right)\left[s_{12} Z_{1 \mathrm{i}}\left(\theta_{2}\right) Z_{1 \dot{2}}\left(\theta_{1}\right)+s_{21} Z_{1 \dot{2}}\left(\theta_{2}\right) Z_{1 \mathrm{i}}\left(\theta_{1}\right)\right] \\
Z_{1 \mathrm{i}}\left(\theta_{1}\right) Z_{2 \dot{2}}\left(\theta_{2}\right)=S\left(\theta_{12}\right)\left[s_{12}^{2} Z_{1 \mathrm{i}}\left(\theta_{2}\right) Z_{2 \dot{2}}\left(\theta_{1}\right)+s_{21}^{2} Z_{2 \dot{2}}\left(\theta_{2}\right) Z_{1 \mathrm{i}}\left(\theta_{1}\right)\right. \\
\left.+s_{12} s_{21}\left(Z_{2 \mathrm{i}}\left(\theta_{2}\right) Z_{1 \dot{2}}\left(\theta_{1}\right)+Z_{1 \dot{2}}\left(\theta_{2}\right) Z_{2 \dot{1}}\left(\theta_{1}\right)\right)\right]
\end{gathered}
$$

It is easy to verify all these relations by using the formulae for the ZF operators from the previous subsection. As was mentioned before, the commutativity of charges follows from the relations $(3.31)$ and $(3.32)$. As an example, let us prove the commutativity of the charge $\chi_{1}^{-}$with $Z_{2 i}$.

One has (dropping $\rho(\theta)$ )

$$
\begin{aligned}
\chi_{1}^{-} Z_{2 \dot{1}}^{(1)}(\theta) & \sim \int_{C_{\alpha_{\dot{r}}} / \theta / \theta^{--} / C_{\alpha_{\dot{q}}}} c_{1 \dot{r}} c_{1 \dot{q}} g_{0 \mid 1 \dot{q}}^{a} g_{0 \mid 1 \dot{r}} g_{1 \dot{q} \mid 1 \dot{r}} e^{i \phi_{0}+i \phi_{1 \dot{r}}+i \phi_{1 \dot{q}}} \\
& =\int_{\theta^{--} / C_{\alpha_{\dot{r}}}=C_{\alpha_{\dot{q}}}} c_{1 \dot{r}} c_{1 \dot{q}} g_{0 \mid 1 \dot{q}}^{a} g_{0 \mid 1 \dot{r}} g_{1 \dot{q} \mid 1 \dot{r}} e^{i \phi_{0}+i \phi_{1 \dot{r}}+i \phi_{1 \dot{q}}} \\
& +\int_{\theta^{--} / C_{\alpha_{\dot{q}}}} c_{1 \dot{r}}(\theta) c_{1 \dot{q}} g_{0 \mid 1 \dot{q}}^{a} R_{0 \mid 1 \dot{r}} g_{1 \dot{q} \mid 1 \dot{r}}\left(\alpha_{1 \dot{q}}-\theta\right) e^{i \phi_{0}+i \phi_{1 \dot{r}}(\theta)+i \phi_{1 \dot{q}}} \\
\chi_{1}^{-} Z_{2 \dot{1}}^{(2)}(\theta) & \sim \int_{C_{\alpha_{\dot{r}}} / \theta} c_{1 \dot{r}} c_{1 \dot{q}}(\theta) R_{0 \mid 1 \dot{q}} g_{0 \mid 1 \dot{r}} g_{1 \dot{q} \mid 1 \dot{r}}\left(\theta-\alpha_{1 \dot{r}}\right) e^{i \phi_{0}+i \phi_{1 \dot{r}}+i \phi_{1 \dot{q}}(\theta)} \\
& =\int_{\theta^{--} / C_{\alpha_{\dot{r}}}} c_{1 \dot{r}} c_{1 \dot{q}}(\theta) R_{0 \mid 1 \dot{q}} g_{0 \mid 1 \dot{r}} g_{1 \dot{q} \mid 1 \dot{r}}\left(\theta-\alpha_{1 \dot{r}}\right) e^{i \phi_{0}+i \phi_{1 \dot{r}}+i \phi_{1 \dot{q}}(\theta)},
\end{aligned}
$$

where one used that $g_{1 \mathrm{i} \mid 1 \mathrm{i}}(0)=0$. One also has

$$
\begin{gathered}
Z_{2 \dot{1}}^{(1)}(\theta) \chi_{1}^{-} \sim \int_{\theta^{--} / C_{\alpha_{\dot{r}}}=C_{\alpha_{\dot{q}}}} c_{1 \dot{r}}\left(\alpha_{\dot{r}}\right) c_{1 \dot{r}} c_{1 \dot{q}} g_{0 \mid 1 \dot{q}}^{a} g_{1 \dot{r} \mid 0} g_{1 \dot{r} \mid 1 \dot{q}} e^{i \phi_{0}+i \phi_{1 \dot{r}}+i \phi_{1 \dot{q}}}, \\
Z_{2 \dot{1}}^{(2)}(\theta) \chi_{1}^{-} \sim \int_{\theta^{--} / C_{\alpha_{\dot{r}}}} c_{1 \dot{r}} c_{1 \dot{q}}(\theta) R_{0 \mid 1 \dot{q}} g_{1 \dot{r} \mid 0} g_{1 \dot{r} \mid 1 \dot{q}}\left(\alpha_{1 \dot{r}}-\theta\right) e^{i \phi_{0}+i \phi_{1 \dot{r}}+i \phi_{1 \dot{q}}(\theta)},
\end{gathered}
$$

where there is no need to shift the integration contour $C_{\alpha_{\dot{r}}}$. It is easy to check that the integrands of the double integrals are equal to each other after symmetrisation with respect to $\dot{r}, \dot{q}$, and that the integrands of the single integrals also add up to 0 . 


\section{$5 \quad$ Bound states}

The ordering of integration contours discussed in the previous section is particularly useful to analyse the highest weight $r$-particle bound state vertex operators 4

$$
Z_{12 \ldots r, \dot{1} \ldots \dot{r}}(\theta)=\lim _{\epsilon_{i+1, i} \rightarrow 0} \prod_{j=2}^{r}\left(i \epsilon_{j+1, j}\right) Z_{1 \dot{1}}\left(\theta_{1}^{\epsilon}\right) Z_{2 \dot{2}}\left(\theta_{2}^{\epsilon}\right) \cdots Z_{r \dot{r}}\left(\theta_{r}^{\epsilon}\right), \quad \theta_{j}^{\epsilon} \equiv \theta+\mathfrak{u}_{r-2 j+1}+\epsilon_{j},
$$

where $\mathfrak{u}_{k} \equiv \frac{\pi}{N} i k$ is purely imaginary, $\epsilon_{1}=0$ and all $\epsilon_{j k} \equiv \epsilon_{j}-\epsilon_{k}$ do not vanish until one takes the limits.

Similarly to bound states of the chiral GN model [27], any rank- $r$ bound state vertex operator is generated from the highest weight vertex operator $Z_{12 \ldots r, \dot{1} \dot{2} \ldots \dot{r}}$ by acting on it with the lowering symmetry operators.

The highest weight multi-particle bound state vertex operators $Z_{12 \ldots r, \dot{i} \ldots \ldots \dot{r}}$ can be found from the terms in ZF operators which have no integration after the integration contours have been reduced to the canonical form.

\section{Highest weight 2-particle bound state}

It is easy to see that the products $Z_{1 \dot{1}}\left(\theta_{1}\right) Z_{2 \dot{2}}\left(\theta_{2}\right), Z_{2 \dot{2}}\left(\theta_{1}\right) Z_{1 \dot{1}}\left(\theta_{2}\right), Z_{2 \dot{1}}\left(\theta_{1}\right) Z_{1 \dot{2}}\left(\theta_{2}\right)$ and $Z_{1 \dot{2}}\left(\theta_{1}\right) Z_{2 \dot{1}}\left(\theta_{2}\right)$ have a pole at $\theta_{12}=2 \pi i / N$ which means that for $N \geq 3$ these operators form a bound state, while for $N=2$ the pole shows that $Z_{1 \dot{1}}, Z_{2 \dot{2}}$, and $Z_{1 \dot{2}}, Z_{2 \dot{1}}$ are particle-antiparticle pairs.

To find $Z_{12, \dot{i} \dot{2}}$ let us consider $Z_{1 \dot{1}}\left(\theta_{1}\right) Z_{2 \dot{2}}\left(\theta_{2}\right)$ in the limit $\theta_{12} \rightarrow \mathfrak{u}_{2}$. Since the canonically-ordered integration contours in $Z_{2 \dot{2}}$ run below all poles and $g_{1 i \mid 0}=g_{11 \mid 0}=1$, only the last term in 4.12 produces the bound state pole. Introducing the notation

$$
U_{2 \dot{2}}(\theta)=\mathcal{N}_{2 \dot{2}}(\theta) e^{i \phi_{0}(\theta)+i \phi_{1 \dot{1}}(\theta)+i \dot{\phi}_{12}(\theta)}, \quad \mathcal{N}_{2 \dot{2}}(\theta)=\rho(\theta) c_{1 \dot{1}}(\theta) c_{\dot{1} 2}(\theta) R_{0 \mid 1 \dot{1}} R_{1 \dot{1} \mid \dot{1} 2},
$$

one finds

$$
\begin{aligned}
& Z_{1 \dot{1}}\left(\theta_{1}\right) U_{2 \dot{2}}\left(\theta_{2}\right)=\rho\left(\theta_{1}\right) \mathcal{N}_{2 \dot{2}}\left(\theta_{2}\right) g_{00}\left(\theta_{21}\right) g_{\mathrm{i} 2 \mid 0}\left(\theta_{21}\right) e^{i \phi_{0}\left(\theta_{1}\right)+i \phi_{0}\left(\theta_{2}\right)+i \phi_{1 \mathrm{i}}\left(\theta_{2}\right)+i \dot{\phi}_{\mathrm{i} 2}\left(\theta_{2}\right)} \\
& =-\frac{g_{00}\left(-\mathfrak{u}_{2}\right) r_{\dot{1} 2 \mid 0}}{\theta_{12}-\mathfrak{u}_{2}} \rho\left(\theta_{1}\right) \mathcal{N}_{2 \dot{2}}\left(\theta_{2}\right) e^{i \phi_{0}\left(\theta_{1}\right)+i \phi_{0}\left(\theta_{2}\right)+i \phi_{1 \dot{1}}\left(\theta_{2}\right)+i \dot{\phi}_{\mathrm{i} 2}\left(\theta_{2}\right)}+\mathcal{O}(1) \\
& =\frac{i}{\theta_{12}-\mathfrak{u}_{2}} Z_{12, \dot{1} \dot{2}}(\theta)+\mathcal{O}(1), \quad \theta_{1} \rightarrow \theta^{+} \equiv \theta+\mathfrak{u}_{1}, \theta_{2} \rightarrow \theta^{-} \equiv \theta-\mathfrak{u}_{1} .
\end{aligned}
$$

Here the highest weight 2-particle bound state vertex operator $Z_{(2)} \equiv Z_{12, \mathrm{i2}}$ is given by

$$
\begin{aligned}
Z_{(2)}(\theta) & =\mathcal{N}_{(2)}(\theta) e^{i \phi_{0}\left(\theta^{+}\right)+i \phi_{0}\left(\theta^{-}\right)+i \phi_{1 \mathrm{i}}\left(\theta^{-}\right)+i \dot{\phi}_{\mathrm{i} 2}\left(\theta^{-}\right)}, \\
\mathcal{N}_{(2)}(\theta) & =i g_{00}\left(-\mathfrak{u}_{2}\right) r_{\dot{1} \mid 0} \rho\left(\theta^{+}\right) \mathcal{N}_{2 \dot{2}}\left(\theta^{-}\right)
\end{aligned}
$$

\footnotetext{
${ }^{4}$ In this section the dotted and undotted indices are identified that is if $r$ is equal to a number then $\dot{r}$ is equal to the same number with a dot, e.g. if $r=3$ then $\dot{r}=\dot{3}$.
} 
Thus, up to a normalisation the vertex operator

$$
V_{(2)}(\theta)=e^{i \phi_{(2)}(\theta)}, \quad \phi_{(2)}(\theta)=\phi_{0}\left(\theta^{+}\right)+\phi_{0}\left(\theta^{-}\right)+\phi_{1 \mathrm{i}}\left(\theta^{-}\right)+\dot{\phi}_{\mathrm{i} 2}\left(\theta^{-}\right),
$$

creates the highest weight 2-particle bound state. All the other 2-particle bound states are obtained from $Z_{(2)}$ by acting on it with the charges $\chi_{\bar{k}}^{-}, \dot{\chi}_{\dot{k}}^{-}$. It is easy to check that the fields $\phi_{1 \dot{r}}$ and $\dot{\phi}_{i r}$ have trivial Green's functions with the 2-particle bound state free field $\left.\phi_{(2)}\right)^{5}$

$$
\left\langle\phi_{1 \dot{r}}(\alpha) \phi_{(2)}(\theta)\right\rangle=\left\langle\dot{\phi}_{1 r}(\alpha) \phi_{(2)}(\theta)\right\rangle=\left\langle\phi_{(2)}(\theta) \phi_{1 \dot{r}}(\alpha)\right\rangle=\left\langle\phi_{(2)}(\theta) \dot{\phi}_{i_{r}}(\alpha)\right\rangle=1 .
$$

Thus, the charges $\chi_{1}^{-}, \dot{\chi}_{\dot{1}}^{-}$commute with $Z_{(2)}$

$$
\left[\chi_{1}^{-}, Z_{(2)}(\theta)\right]=\left[\dot{\chi}_{\dot{1}}^{-}, Z_{(2)}(\theta)\right]=0,
$$

which is a necessary condition for $Z_{(2)}$ to be the highest weight 2-particle bound state.

For $N=2$ the operators $Z_{1 i}$ and $Z_{22}$ form a particle-antiparticle pair, and the operator $Z_{(2)}(\theta)$ must be equal to $-C_{1 \dot{1}, 2 \dot{2}}=-1$ where $C_{k i, m \dot{n}}=\epsilon_{k m} \epsilon_{\dot{i n}}$ is the charge conjugation matrix. Thus, one finds the following relation between the free fields

$$
\phi_{0}\left(\theta^{++}\right)+\phi_{0}(\theta)+\phi_{1 \dot{1}}(\theta)+\dot{\phi}_{12}(\theta)=0
$$

which together with

$$
\phi_{1 \dot{1}}(\theta)+\dot{\phi}_{12}(\theta)=\phi_{1 \dot{2}}(\theta)+\dot{\phi}_{11}(\theta),
$$

reduces the number of independent fields to 3 . Then, the normalisation condition $\mathcal{N}_{(2)}(\theta)=-1$ leads to

$$
g_{00}\left(-\mathfrak{u}_{2}\right) R_{\mathrm{i} 2 \mid 0} R_{0 \mid 1 \mathrm{i}} R_{1 \mathrm{i} \mid \mathrm{i} 2} \rho\left(\theta^{+}\right) \rho\left(\theta^{-}\right) c_{1 \mathrm{i}}\left(\theta^{-}\right) c_{12}\left(\theta^{-}\right)=-1 .
$$

Taking into account that for $N=2$

$$
g_{00}\left(-\mathfrak{u}_{2}\right)=\frac{2}{\pi}, \quad R_{\mathrm{i} 2 \mid 0}=R_{0 \mid 1 \mathrm{i}}=R_{1 \mathrm{i} \mid \mathrm{i} 2}=\pi,
$$

one gets

$$
2 \pi^{2} \rho\left(\theta^{++}\right) \rho(\theta) c_{1 \mathrm{i}}(\theta) c_{\mathrm{i} 2}(\theta)=-1 .
$$

Now, according to 3.38

$$
\rho(\theta)=e^{i \varkappa \theta} \tilde{\rho}, \quad c_{k \dot{r}}(\theta)=e^{i \varkappa_{k} \theta} \tilde{c}_{k \dot{r}}, \quad c_{\dot{k} r}(\theta)=e^{i \varkappa_{\dot{k}} \theta} \tilde{c}_{\dot{k} r},
$$

and therefore the equation 5.12 splits into the following two relations

$$
2 \varkappa+\varkappa_{1}+\varkappa_{1}=0, \quad 2 \pi^{2} e^{-\pi \varkappa} \tilde{\rho}^{2} \tilde{c}_{1 \mathrm{i}} \tilde{c}_{12}=-1 .
$$

\footnotetext{
${ }^{5}$ Obviously, $\phi_{k \dot{r}}$ and $\dot{\phi}_{k r}$ for $k \geq 3$ also have trivial Green's functions with $\phi_{(2)}$.
} 
These two relations together with the equation (3.32) provide 3 constraints on the 8 parameters. In addition, it is clear that for $N=2$ the extended ZF algebra is invariant under the following scaling transformations

$$
Z_{1 \mathrm{i}} \rightarrow \sigma_{1 \mathrm{i}} Z_{1 \mathrm{i}}, \quad \chi_{1} \rightarrow \sigma_{1} \chi_{1}, \quad \dot{\chi}_{\mathrm{i}} \rightarrow \frac{1}{\sigma_{1 \mathrm{i}}^{2} \sigma_{1}} \dot{\chi}_{\mathrm{i}}
$$

where $\sigma_{11}$ and $\sigma_{1}$ are arbitrary constants. Thus, one can set $\tilde{c}_{11}$ and $\tilde{c}_{12}$ to any values, and therefore the number of independent parameters leading to physically distinct results is 3 which agrees with [19]. For example, choosing $\tilde{c}_{1 i}=e^{-\pi \varkappa_{1} / 2}, \tilde{c}_{i 2}=e^{-\pi \varkappa_{i} / 2}$, one gets $\tilde{\rho}=\frac{i}{\sqrt{2} \pi}$, and the following two constraints on the remaining five parameters

$$
2 \varkappa+\varkappa_{1}+\varkappa_{i}=0, \quad e^{-\pi \varkappa} \tilde{c}_{12} \tilde{c}_{11}=1 .
$$

\section{Highest weight 3-particle bound state}

As was mentioned above, the 2-particle bound state field $\phi_{(2)}$ has trivial Green's functions with $\phi_{k \dot{r}}, \dot{\phi}_{\dot{k} r}$ for $k \neq 2, \dot{k} \neq \dot{2}$. It is easy to find that it has the following Green's functions with $\phi_{0}, \phi_{2 \dot{q}}, \dot{\phi}_{\dot{2} q}$

$$
\begin{gathered}
g_{0(2)}(\theta)=g_{(2) 0}(\theta)=\frac{\Gamma\left(\frac{i \theta}{2 \pi}-\frac{3}{2 N}+1\right) \Gamma\left(\frac{i \theta}{2 \pi}+\frac{3}{2 N}\right)}{\Gamma\left(\frac{i \theta}{2 \pi}-\frac{1}{2 N}+1\right) \Gamma\left(\frac{i \theta}{2 \pi}+\frac{1}{2 N}\right)}, \\
g_{2 \dot{q} \mid(2)}(\theta)=g_{\dot{2} q \mid(2)}(\theta)=\frac{\theta+\mathfrak{u}_{1}}{\theta+\mathfrak{u}_{3}}, \quad g_{(2) \mid 2 \dot{q}}(\theta)=g_{(2) \mid \dot{2} q}(\theta)=1, \quad q \geq 3, \dot{q} \geq \dot{3}, \\
g_{2 \dot{q} \mid(2)}(\theta)=g_{\dot{2} q \mid(2)}(\theta)=1, \quad g_{(2) \mid 2 \dot{q}}(\theta)=g_{(2) \mid \dot{2} q}(\theta)=\frac{\theta-\mathfrak{u}_{3}}{\theta-\mathfrak{u}_{1}}, \quad q \leq 2, \dot{q} \leq \dot{2} .
\end{gathered}
$$

Thus, one can get a pole in $Z_{12, \dot{1} \dot{2}}\left(\theta_{1}\right) Z_{3 \dot{3}}\left(\theta_{2}\right)$ at $\theta_{12}=-\mathfrak{u}_{3}$ only if $Z_{3 \dot{3}}\left(\theta_{2}\right)$ contains a term with $\phi_{2 \dot{3}}$ or $\phi_{\dot{2} 3}$. The terms in $Z_{3 \dot{3}}$ without integration are obtained from

$$
\dot{\chi}_{\dot{2}}^{-} \chi_{2}^{-} U_{2 \dot{2}}=\mathcal{N}_{2 \dot{2}}(\theta) \int c_{2 \dot{r}} c_{\dot{2} q} g_{1 \dot{\mathrm{i}} \mid 2 \dot{r}} g_{\dot{1} 2 \mid 2 \dot{r}} g_{1 \dot{1} \mid \dot{2} q} g_{\dot{1} 2 \mid \dot{2} q} g_{2 \dot{r} \mid \dot{2} q} e^{i \phi_{0}(\theta)+i \phi_{1 \dot{1}}(\theta)+i \dot{\phi}_{\dot{1} 2}(\theta)+i \phi_{2 \dot{r}}+i \dot{\phi}_{\dot{2} q}} \text {. }
$$

If $\dot{r}=\dot{3}$ then the function $g_{1 \mathrm{i} \mid 2 \dot{r}} g_{\mathrm{i} 2 \mid 2 \dot{r}}$ has no pole, and taking the contour $C_{2 \dot{r}}$ below $\theta^{--}$ would not produce any term without integration. Thus, $q=3$ and therefore $\dot{r}=\dot{2}$, and the term of interest is

$$
\begin{aligned}
U_{3 \dot{3}}(\theta) & =\mathcal{N}_{3 \dot{3}}(\theta) e^{i \phi_{0}(\theta)+i \phi_{1 \dot{1}}(\theta)+i \dot{\phi}_{12}(\theta)+i \phi_{2 \dot{2}}(\theta)+i \dot{\phi}_{\dot{2} 3}(\theta)}, \\
\mathcal{N}_{3 \dot{3}}(\theta) & =\mathcal{N}_{2 \dot{2}}(\theta) c_{2 \dot{2}}(\theta) c_{\dot{2} 3}(\theta) R_{\dot{1} 2 \mid 2 \dot{2}} R_{2 \dot{2} \mid \dot{2} 3} .
\end{aligned}
$$

Then, the highest weight 3 -particle bound state vertex operator $Z_{(3)} \equiv Z_{123, \dot{i} \dot{3} \text { appears }}$ in the residue of the product $Z_{(2)}\left(\theta_{1}\right) U_{3 \dot{3}}\left(\theta_{2}\right)$ at $\theta_{12}=3 \pi i / N$

$$
\begin{aligned}
& Z_{(3)}(\theta)=\lim _{\epsilon \rightarrow 0} i \epsilon Z_{(2)}\left(\theta+\mathfrak{u}_{1}\right) U_{3 \dot{3}}\left(\theta-\mathfrak{u}_{2}+\epsilon\right) \\
& =\mathcal{N}_{(3)}(\theta) e^{i \phi_{(2)}\left(\theta^{+}\right)+i \phi_{0}\left(\theta^{--}\right)+i \phi_{1 \dot{1}}\left(\theta^{--}\right)+i \dot{\phi}_{\dot{1} 2}\left(\theta^{--}\right)+i \phi_{2 \dot{2}}\left(\theta^{--}\right)+i \dot{\phi}_{\dot{2} 3}\left(\theta^{--}\right)} \\
& \mathcal{N}_{(3)}(\theta)=i g_{0(2)}\left(-\mathfrak{u}_{3}\right) r_{2 \dot{3} \mid(2)} \mathcal{N}_{(2)}\left(\theta+\mathfrak{u}_{1}\right) \mathcal{N}_{3 \dot{3}}\left(\theta-\mathfrak{u}_{2}\right) .
\end{aligned}
$$


Thus, up to a normalisation the vertex operator

$$
\begin{aligned}
V_{(3)}(\theta) & =e^{i \phi_{(3)}(\theta)} \\
\phi_{(3)}(\theta) & =\phi_{0}\left(\theta^{++}\right)+\phi_{0}(\theta)+\phi_{0}\left(\theta^{--}\right)+\phi_{1 \dot{1}}(\theta)+\phi_{1 \dot{1}}\left(\theta^{--}\right) \\
& +\dot{\phi}_{12}(\theta)+\dot{\phi}_{12}\left(\theta^{--}\right)+\phi_{2 \dot{2}}\left(\theta^{--}\right)+\dot{\phi}_{\dot{2} 3}\left(\theta^{--}\right)
\end{aligned}
$$

creates the highest weight 3-particle bound state for $N>3$, while for $N=3$ the operator $Z_{(2)}$ creates the anti-particle of $Z_{3 \dot{3}}$, and therefore $V_{(3)}$ must be equal to 1

$$
V_{(3)}(\theta)=1, \quad N=3
$$

leading to the relation

$$
\begin{aligned}
\phi_{0}\left(\theta^{++}\right) & +\phi_{0}(\theta)+\phi_{0}\left(\theta^{--}\right)+\phi_{1 \dot{1}}(\theta)+\phi_{1 \dot{1}}\left(\theta^{--}\right) \\
& +\dot{\phi}_{12}(\theta)+\dot{\phi}_{\dot{1} 2}\left(\theta^{--}\right)+\phi_{2 \dot{2}}\left(\theta^{--}\right)+\dot{\phi}_{\dot{2} 3}\left(\theta^{--}\right)=0, \quad N=3 .
\end{aligned}
$$

Then, the normalisation condition $\mathcal{N}_{(3)}(\theta)=-1$ leads again to two equations on the parameters $\varkappa, \varkappa_{k}, \varkappa_{\dot{k}}, \tilde{c}_{k \dot{r}}, \tilde{c}_{k r}$ which will be discussed later for the general $N$ case.

\section{Highest weight 4-particle bound state}

It is easy to verify that the 3-particle bound state field $\phi_{(3)}$ has trivial Green's functions with $\phi_{k \dot{r}}, \phi_{k r}$ for $k \neq 3, \dot{k} \neq \dot{3}$, and it has the following Green's functions with $\phi_{0}, \phi_{3 \dot{q}}$, $\phi_{\dot{3} q}$

$$
\begin{gathered}
g_{0(3)}(\theta)=g_{(3) 0}(\theta)=\frac{\Gamma\left(\frac{i \theta}{2 \pi}-\frac{2}{N}+1\right) \Gamma\left(\frac{i \theta}{2 \pi}+\frac{2}{N}\right)}{\Gamma\left(\frac{i \theta}{2 \pi}-\frac{1}{N}+1\right) \Gamma\left(\frac{i \theta}{2 \pi}+\frac{1}{N}\right)}, \\
g_{3 \dot{q} \mid(3)}(\theta)=g_{\dot{3} q \mid(3)}(\theta)=\frac{\theta+\mathfrak{u}_{2}}{\theta+\mathfrak{u}_{4}}, \quad g_{(3) \mid 3 \dot{q}}(\theta)=g_{(3) \mid \dot{3} q}(\theta)=1, \quad q \geq 4, \dot{q} \geq \dot{4}, \\
g_{3 \dot{q} \mid(3)}(\theta)=g_{\dot{3} q \mid(3)}(\theta)=1, \quad g_{(3) \mid 3 \dot{q}}(\theta)=g_{(3) \mid \dot{3} q}(\theta)=\frac{\theta-\mathfrak{u}_{4}}{\theta-\mathfrak{u}_{2}}, \quad q \leq 3, \dot{q} \leq \dot{3} .
\end{gathered}
$$

Thus, one can get a pole in $Z_{123, \dot{i} \dot{\dot{j}}}\left(\theta_{1}\right) Z_{4 \dot{4}}\left(\theta_{2}\right)$ at $\theta_{12}=-\mathfrak{u}_{4}$ only if $Z_{4 \dot{4}}\left(\theta_{2}\right)$ contains a term with $\phi_{3 \dot{4}}$ or $\phi_{\dot{3} 4}$. The terms in $Z_{4 \dot{4}}$ without integration are obtained from

$$
\begin{aligned}
\dot{\chi}_{\dot{3}}^{-} \chi_{3}^{-} U_{3 \dot{3}}=\mathcal{N}_{3 \dot{3}}(\theta) \int & c_{3 \dot{r}} c_{\dot{3} q} g_{2 \dot{2} \mid 3 \dot{r}} g_{\dot{2} 3 \mid 3 \dot{r}} g_{2 \dot{2} \mid \dot{3} q} g_{\dot{2} 3 \mid \dot{3} q} g_{3 \dot{r} \mid \dot{3} q} \\
& \times e^{i \phi_{0}(\theta)+i \phi_{1 \dot{1}}(\theta)+i \dot{\phi}_{\dot{1} 2}(\theta)+i \phi_{2 \dot{2}}(\theta)+i \dot{\phi}_{\dot{2} 3}(\theta)+i \phi_{3 \dot{r}}+i \dot{\phi}_{\dot{3} q}}
\end{aligned}
$$

and the term without integration is obtained for $q=4$ and $\dot{r}=\dot{3}$

$$
\begin{aligned}
U_{4 \dot{4}}(\theta) & =\mathcal{N}_{4 \dot{4}}(\theta) e^{i \phi_{0}(\theta)+i \phi_{1 \dot{1}}(\theta)+i \dot{\phi}_{\dot{1} 2}(\theta)+i \phi_{2 \dot{2}}(\theta)+i \dot{\phi}_{\dot{2} 3}(\theta)+i \phi_{3 \dot{3}}(\theta)+i \dot{\phi}_{\dot{3} 4}(\theta)}, \\
\mathcal{N}_{4 \dot{4}}(\theta) & =\mathcal{N}_{3 \dot{3}}(\theta) c_{3 \dot{3}}(\theta) c_{\dot{3} 4}(\theta) R_{\dot{2} 3 \mid 3 \dot{3}} R_{3 \dot{3} \mid \dot{3} 4} .
\end{aligned}
$$


Then, the highest weight 4-particle bound state vertex operator $Z_{(4)} \equiv Z_{1234, \dot{1} \dot{2} \dot{3} \dot{4}}$ appears in the residue of the product $Z_{(3)}\left(\theta_{1}\right) U_{4 \dot{4}}\left(\theta_{2}\right)$ at $\theta_{12}=4 \pi i / N$

$$
\begin{aligned}
& Z_{(4)}(\theta)=\lim _{\epsilon \rightarrow 0} i \epsilon \mathcal{Z}_{(3)}\left(\theta+\mathfrak{u}_{1}\right) U_{4 \dot{4}}\left(\theta-\mathfrak{u}_{3}+\epsilon\right)=\mathcal{N}_{(4)}(\theta) e^{i \phi_{(4)}(\theta)}, \\
& \mathcal{N}_{(4)}(\theta)=i g_{0(3)}\left(-\mathfrak{u}_{4}\right) r_{3 \dot{4} \mid(3)} \mathcal{N}_{(3)}\left(\theta+\mathfrak{u}_{1}\right) \mathcal{N}_{4 \dot{4}}\left(\theta-\mathfrak{u}_{3}\right), \\
& \phi_{(4)}(\theta)=\sum_{k=-\frac{3}{2}}^{\frac{3}{2}} \phi_{0}\left(\theta+\mathfrak{u}_{2 k}\right)+\sum_{n=1}^{3} \sum_{k=-\frac{3}{2}}^{\frac{3}{2}-n}\left(\phi_{n \dot{n}}\left(\theta+\mathfrak{u}_{2 k}\right)+\phi_{\dot{n}, n+1}\left(\theta+\mathfrak{u}_{2 k}\right)\right) .
\end{aligned}
$$

Thus, up to a normalisation the vertex operator $V_{(4)}(\theta)=e^{i \phi_{(4)}(\theta)}$ creates the highest weight 4-particle bound state for $N>4$, while for $N=4$ the operator $Z_{(3)}$ creates the anti-particle of $Z_{4 \dot{4}}$, and therefore $V_{(4)}$ satisfies

$$
V_{(4)}(\theta)=1, \quad N=4 .
$$

Moreover, for any $N$ the 4-particle bound state field $\phi_{(4)}$ has trivial Green's functions with with $\phi_{k \dot{r}}, \phi_{k r}$ for $k \neq 4, \dot{k} \neq \dot{4}$.

\section{Highest weight $r$-particle bound state}

The formulae above can be easily generalised, and the highest weight $r$-particle bound state vertex operator $Z_{(r)} \equiv Z_{12 \ldots r, \mathrm{i} \dot{2} \ldots \dot{r}}$ is given by

$$
\begin{aligned}
& Z_{(r)}(\theta)=\lim _{\epsilon \rightarrow 0} i \epsilon \mathcal{Z}_{(r-1)}\left(\theta+\mathfrak{u}_{1}\right) U_{r \dot{r}}\left(\theta-\mathfrak{u}_{r-1}+\epsilon\right)=\mathcal{N}_{(r)}(\theta) e^{i \phi_{(r)}(\theta)}, \\
& \mathcal{N}_{(r)}(\theta)=i g_{0(r-1)}\left(-\mathfrak{u}_{r}\right) r_{r-1, \dot{r}} \mid(r-1) \\
& \mathcal{N}_{(r-1)}\left(\theta+\mathfrak{u}_{1}\right) \mathcal{N}_{r \dot{r}}\left(\theta-\mathfrak{u}_{r-1}\right), \\
& \mathcal{N}_{r \dot{r}}(\theta)=\mathcal{N}_{r-1, \dot{r}-\dot{1}}(\theta) c_{r-1, \dot{r}-\dot{1}}(\theta) c_{\dot{r}-\dot{1}, r}(\theta) R_{\dot{r}-\dot{2}, r-1 \mid r-1, \dot{r}-\dot{1}} R_{r-1, \dot{r}-\dot{1} \mid \dot{r}-\dot{1} r}, \\
& \phi_{(r)}(\theta)=\sum_{k=-\frac{r-1}{2}}^{\frac{r-1}{2}} \phi_{0}\left(\theta+\mathfrak{u}_{2 k}\right)+\sum_{n=1}^{r-1} \sum_{k=-\frac{r-1}{2}}^{\frac{r-1}{2}-n}\left(\phi_{n \dot{n}}\left(\theta+\mathfrak{u}_{2 k}\right)+\phi_{\dot{n}, n+1}\left(\theta+\mathfrak{u}_{2 k}\right)\right) .
\end{aligned}
$$

The $r$-particle bound state field $\phi_{(r)}$ commutes with $\phi_{k \dot{r}}, \phi_{k r}$ for $k \neq r, \dot{k} \neq \dot{r}$, and it has the following Green's functions with $\phi_{0}, \phi_{r \dot{q}}, \phi_{\dot{r} q}$

$$
\begin{gathered}
g_{0(r)}(\theta)=g_{(r) 0}(\theta)=\frac{\Gamma\left(\frac{i \theta}{2 \pi}-\frac{r+1}{2 N}+1\right) \Gamma\left(\frac{i \theta}{2 \pi}+\frac{r+1}{2 N}\right)}{\Gamma\left(\frac{i \theta}{2 \pi}-\frac{r-1}{2 N}+1\right) \Gamma\left(\frac{i \theta}{2 \pi}+\frac{r-1}{2 N}\right)}, \\
g_{0(r)}\left(-\mathfrak{u}_{r+1}\right)=\frac{r \Gamma\left(\frac{r+1}{N}\right)}{\Gamma\left(\frac{1}{N}\right) \Gamma\left(1+\frac{r}{N}\right)}, \\
g_{r \dot{q} \mid(r)}(\theta)=g_{\dot{r} q \mid(r)}(\theta)=\frac{\theta+\mathfrak{u}_{r-1}}{\theta+\mathfrak{u}_{r+1}}, \quad g_{(r) \mid r \dot{q}}(\theta)=g_{(r) \mid \dot{r} q}(\theta)=1, \quad \dot{q}>\dot{r}, \\
g_{r \dot{q} \mid(r)}(\theta)=g_{\dot{r} q \mid(r)}(\theta)=1, \quad g_{(r) \mid r \dot{q}}(\theta)=g_{(r) \mid \dot{r} q}(\theta)=\frac{\theta-\mathfrak{u}_{r+1}}{\theta-\mathfrak{u}_{r-1}}, \quad \dot{q} \leq \dot{r} .
\end{gathered}
$$


Explicit formulae expressing $\phi_{0}$ and $\phi_{(r)}$ in terms of the elementary fields are collected in appendix $\mathrm{B}$.

The normalisation functions $\mathcal{N}_{(r)}(\theta)$ can be found explicitly. First, one expresses them in terms of $\mathcal{N}_{k \dot{k}}$

$$
\mathcal{N}_{(r)}(\theta)=R_{0 \mid 1 \mathrm{i}}^{r-1} \prod_{k=1}^{r-1} g_{0(k)}\left(-\mathfrak{u}_{k+1}\right) \prod_{k=1}^{r} \mathcal{N}_{k \dot{k}}\left(\theta-\mathfrak{u}_{2 k-r-1}\right)
$$

where $\mathcal{N}_{1 \mathrm{i}}(\theta) \equiv \rho(\theta)$, and one takes into account that the residues of all the functions in the formula (5.33) are equal to $r_{0 \mid 1 i}=-2 \pi i / N$. Next, one gets

$$
\begin{gathered}
\mathcal{N}_{k \dot{k}}(\theta)=\rho(\theta) R_{0 \mid 1 \dot{1}}^{2(k-1)} \prod_{m=1}^{k-1} c_{m \dot{m}}(\theta) c_{\dot{m}, m+1}(\theta), \\
\prod_{k=1}^{r-1} g_{0(k)}\left(-\mathfrak{u}_{k+1}\right)=\frac{\Gamma\left(\frac{r}{N}\right)}{\Gamma\left(\frac{1}{N}\right) \Gamma\left(1+\frac{1}{N}\right)^{r-1}},
\end{gathered}
$$

and therefore

$$
\begin{aligned}
\mathcal{N}_{(r)}(\theta) & =\frac{\Gamma\left(\frac{r}{N}\right)}{\Gamma\left(\frac{1}{N}\right) \Gamma\left(1+\frac{1}{N}\right)^{r-1}} R_{0 \mid 1 \dot{1}}^{r^{2}-1} \prod_{k=1}^{r} \rho\left(\theta-\mathfrak{u}_{2 k-r-1}\right) \\
& \times \prod_{k=1}^{r} \prod_{m=1}^{k-1} c_{m \dot{m}}\left(\theta-\mathfrak{u}_{2 k-r-1}\right) c_{\dot{m}, m+1}\left(\theta-\mathfrak{u}_{2 k-r-1}\right)
\end{aligned}
$$

Finally, taking into account (3.38), one gets

$$
\begin{aligned}
\mathcal{N}_{(r)}(\theta) & =\frac{\Gamma\left(\frac{r}{N}\right)}{\Gamma\left(\frac{1}{N}\right) \Gamma\left(1+\frac{1}{N}\right)^{r-1}}\left(\frac{2 \pi}{N}\right)^{r^{2}-1} \tilde{\rho}^{r} e^{i r \varkappa \theta} \prod_{k=1}^{r-1} e^{i(r-k)\left(\varkappa_{k}+\varkappa_{\dot{k}}\right) \theta} \\
& \times \prod_{m=1}^{r-1}\left(e^{\frac{\pi}{N} m \varkappa_{m}} \tilde{c}_{m \dot{m}}\right)^{r-m}\left(e^{\frac{\pi}{N} m \varkappa_{\dot{m}}} \tilde{c}_{\dot{m}, m+1}\right)^{r-m} .
\end{aligned}
$$

The vertex operator $Z_{(N-1)}$ creates the anti-particle of $Z_{N \dot{N}}$, and therefore $Z_{(N)}$ must be equal to -1 . This leads to the relation $\phi_{(N)}=0$, which allows one to express $\phi_{0}$ in terms of the other fields

$$
\sum_{k=-\frac{N-1}{2}}^{\frac{N-1}{2}} \phi_{0}\left(\theta+\mathfrak{u}_{2 k}\right)+\sum_{n=1}^{N-1} \sum_{k=-\frac{N-1}{2}}^{\frac{N-1}{2}-n}\left(\phi_{n \dot{n}}\left(\theta+\mathfrak{u}_{2 k}\right)+\phi_{\dot{n}, n+1}\left(\theta+\mathfrak{u}_{2 k}\right)\right)=0,
$$

and to the normalisation condition

$$
\mathcal{N}_{(N)}(\theta)=-1
$$


It is important to stress that (5.42) leads to the following nontrivial crossing-type equation for Green's function $g_{00}$

$$
\prod_{k=-\frac{N-1}{2}}^{\frac{N-1}{2}} g_{00}\left(\theta+\mathfrak{u}_{2 k}\right)=\frac{\theta+\left(1-\frac{1}{N}\right) \pi i}{\theta-\left(1-\frac{1}{N}\right) \pi i},
$$

which is indeed satisfied.

By using (5.41), the normalisation condition takes the form

$$
\begin{aligned}
\mathcal{N}_{(N)}(\theta) & =\frac{1}{\Gamma\left(\frac{1}{N}\right) \Gamma\left(1+\frac{1}{N}\right)^{N-1}}\left(\frac{2 \pi}{N}\right)^{N^{2}-1} \tilde{\rho}^{N} e^{i N \varkappa \theta} \prod_{k=1}^{N-1} e^{i(N-k)\left(\varkappa_{k}+\varkappa_{\dot{k}}\right) \theta} \\
& \times \prod_{m=1}^{N-1}\left(e^{\frac{\pi}{N} m \varkappa_{m}} \tilde{c}_{m \dot{m}}\right)^{N-m}\left(e^{\frac{\pi}{N} m \varkappa_{m}} \tilde{c}_{\dot{m}, m+1}\right)^{N-m}=-1
\end{aligned}
$$

which splits into the following two equations

$$
\begin{aligned}
& N \varkappa+\sum_{k=1}^{N-1}(N-k)\left(\varkappa_{k}+\varkappa_{\dot{k}}\right)=0, \\
& \frac{1}{\Gamma\left(\frac{1}{N}\right) \Gamma\left(1+\frac{1}{N}\right)^{N-1}}\left(\frac{2 \pi}{N}\right)^{N^{2}-1} \tilde{\rho}^{N} \prod_{m=1}^{N-1}\left(e^{\frac{\pi}{N} m \varkappa_{m}} \tilde{c}_{m \dot{m}}\right)^{N-m}\left(e^{\frac{\pi}{N} m \varkappa_{m}} \tilde{c}_{\dot{m}, m+1}\right)^{N-m}=-1 .
\end{aligned}
$$

Adding these two constraints to the $(N-1)^{2}$ constraints on $\tilde{c}_{k \dot{m}}$ and $\tilde{c}_{\dot{m} k}$, one gets $N^{2}-2 N+3$ constraints on the $2 N^{2}$ parameters $\varkappa, \varkappa_{k}, \varkappa_{k}, \tilde{\rho}, \tilde{c}_{k \dot{r}}, \tilde{c}_{k r}$. The number of physically inequivalent parameters is however less then $N^{2}+2 N-3$ because the extended ZF algebra is invariant under the following scaling transformations

$$
Z_{1 \mathrm{i}} \rightarrow \sigma_{1 \mathrm{i}} Z_{1 \mathrm{i}}, \quad \chi_{k} \rightarrow \sigma_{k} \chi_{k}, \quad \dot{\chi}_{\dot{k}} \rightarrow \sigma_{\dot{k}} \dot{\chi}_{\dot{1}}
$$

where $\sigma_{11}, \sigma_{k}$ and $\sigma_{\dot{k}}$ are constants satisfying the constraint

$$
\sigma_{11}^{N} \prod_{m=1}^{N-1}\left(\sigma_{m} \sigma_{\dot{m}}\right)^{N-m}=1
$$

Thus, one can choose the values of $2 N-2$ parameters, and the number of independent parameters leading to physically distinct results is $N^{2}-1$ which is equal to the number of fields in the $\mathrm{SU}(\mathrm{N}) \times \mathrm{SU}(\mathrm{N}) \mathrm{PCF}$ model. For example, choosing

$$
\tilde{c}_{m \dot{m}}=e^{-\frac{\pi}{N} m \varkappa_{m}}, \quad \tilde{c}_{\dot{m}, m+1}=e^{-\frac{\pi}{N} m \varkappa_{\dot{m}}},
$$

one finds $\tilde{\rho}$

$$
\tilde{\rho}^{N}=-\Gamma\left(\frac{1}{N}\right) \Gamma\left(1+\frac{1}{N}\right)^{N-1}\left(\frac{N}{2 \pi}\right)^{N^{2}-1}
$$


To conclude this section, it is worth mentioning that the normalisation condition $\mathcal{N}_{(N)}=-1$ allows one to obtain the following expression for $\mathcal{N}_{(N-1)}$

$$
\begin{aligned}
\mathcal{N}_{(N-1)}(\theta) & =\Gamma\left(1-\frac{1}{N}\right) \Gamma\left(1+\frac{1}{N}\right)\left(\frac{2 \pi}{N}\right)^{1-2 N} \frac{1}{\tilde{\rho}} e^{-i \varkappa \theta} e^{-i(N-1)\left(\varkappa_{N-1}+\varkappa_{\dot{N}-\dot{1}}\right) \theta} \\
& \times \prod_{m=1}^{r-1}\left(e^{\frac{\pi}{N} m \varkappa_{m}} \tilde{c}_{m \dot{m}}\right)^{-1}\left(e^{\frac{\pi}{N} m \varkappa_{\dot{m}}} \tilde{c}_{\dot{m}, m+1}\right)^{-1}
\end{aligned}
$$

\section{Form factors}

According to Lukyanov [12], up to an overall normalisation constant the form factors of the exponential operator $O$ corresponding to the constructed representation $\pi_{O}$ of the $\mathrm{ZF}$ algebra are

$$
F_{\mathbb{I}_{1} \ldots \mathbb{I}_{n}}^{O}\left(\theta_{1}, \ldots, \theta_{n}\right)=\left\langle\left\langle Z_{\mathbb{I}_{n}}\left(\theta_{n}\right) \cdots Z_{\mathbb{I}_{1}}\left(\theta_{1}\right)\right\rangle\right\rangle
$$

where for any operator $W$ acting in $\pi_{O}$ the quantity $\langle\langle W\rangle\rangle$ is defined by

$$
\langle\langle W\rangle\rangle=\frac{\operatorname{Tr}_{\pi_{O}}\left[e^{2 \pi i \mathbb{K}} W\right]}{\operatorname{Tr}_{\pi_{O}}\left[e^{2 \pi i \mathbb{K}}\right]} .
$$

Due to the $\mathrm{SU}(\mathrm{N}) \times \mathrm{SU}(\mathrm{N})$ symmetry the form factors do not vanish only for the states which do not carry charges with respect to the Cartan generators $P_{k}$ and $P_{\dot{k}}$.

It is clear that the form factors (6.1) are sums of multiple integrals with integrands of the form

$$
R_{\mu_{1} \ldots \mu_{q}}\left(\beta_{1}, \ldots, \beta_{q}\right)=\left\langle\left\langle V_{\mu_{q}}\left(\beta_{q}\right) \cdots V_{\mu_{1}}\left(\beta_{1}\right)\right\rangle\right\rangle,
$$

where the set $\left\{\beta_{1}, \ldots, \beta_{q}\right\}$ contains $\theta_{j}$-rapidities. It is shown in appendix D that for any operator $W$ which is the product of free field exponents

$$
W=V_{n}\left(\theta_{n}\right) \cdots V_{1}\left(\theta_{1}\right), \quad V_{j}(\theta)=: e^{i \phi_{j}(\theta)}:=e^{i \phi_{j}^{+}(\theta)} e^{i \phi_{j}^{-}(\theta)}
$$

one obtains $\langle\langle W\rangle\rangle$ by applying the Wick theorem

$$
\left\langle\left\langle V_{n}\left(\theta_{n}\right) \cdots V_{1}\left(\theta_{1}\right)\right\rangle\right\rangle=\prod_{j=1}^{n} C_{V_{j}} \prod_{k<j} G_{V_{k} V_{j}}\left(\theta_{k}-\theta_{j}\right),
$$

where

$$
\begin{gathered}
C_{V_{j}}=\left\langle\left\langle V_{j}\left(\theta_{j}\right)\right\rangle\right\rangle=\exp \left(-\left\langle\left\langle\phi_{j}^{-}(0) \phi_{j}^{+}(0)\right\rangle\right\rangle\right), \\
G_{V_{k} V_{j}}\left(\theta_{k}-\theta_{j}\right)=\exp \left(-\left\langle\left\langle\phi_{j}\left(\theta_{j}\right) \phi_{k}\left(\theta_{k}\right)\right\rangle\right\rangle\right) .
\end{gathered}
$$

The constants $C_{V_{\mu}}$ and the functions $G_{\mu \nu} \equiv G_{V_{\mu} V_{\nu}}$ are computed in appendix D. It is worth mentioning that some of the functions $G_{\mu \nu}$ are minimal two-particle form factors. In particular, $G_{(N-1)(1)}$ (D.31) is the particle-antiparticle minimal form factor which determines the two-particle form factor of the current operator calculated in [40]. 
The integration contours in 6.1 are similar to the ones for the vacuum expectation values

$$
\left\langle 0\left|Z_{M_{n}}\left(\theta_{n}\right) \cdots Z_{M_{1}}\left(\theta_{1}\right)\right| 0\right\rangle
$$

However, $G_{\mu \mid \nu}$-functions have more poles, and the rule for choosing an integration contour is modified as follows. In addition to the usual requirements, one also requires that an integration contour $C_{A}$ due to the operator $\chi=\int_{C} e^{i \phi_{A}}$ is in the simply-connected region which contains all the poles of $g_{\mu \mid A}$-functions due to the vertex operators $V_{\mu}$ to the right of $\chi$, and all the poles of $g_{A \mid \nu}$-functions due to the vertex operators $V_{\nu}$ to the left of $\chi$ but no other poles of $G_{\mu \mid A}$ and $G_{A \mid \nu}$. For example, the integration contour $C_{k \dot{r}}$ in $\chi_{k}^{-}$runs from $\operatorname{Re} \alpha_{k \dot{r}}=-\infty$ to $\operatorname{Re} \alpha_{k \dot{r}}=+\infty$, and it lies above a pole of the $g_{\mu \mid k \dot{r}}$-function due to the vertex operator $V_{\mu}$ to the right of $\chi_{k}^{-}$and above all the poles of the $G_{\mu \mid k \dot{r}}$-function which are below this pole of $g_{\mu \mid k r}$. However, $C_{k \dot{r}}$ runs below all the poles of the $G_{\mu \mid k \dot{r}}$-function which are above this pole of $g_{\mu \mid k \dot{r}}$. If $g_{\mu \mid k \dot{r}}$ has no pole then $C_{k \dot{r}}$ just runs below all the poles of the $G_{\mu \mid k \dot{r}}$-function. Similarly, the contour $C_{k \dot{r}}$ runs below a pole of the $g_{k \dot{r} \mid \nu}$-function due to the vertex operator $V_{\nu}$ to the left of $\chi_{k}^{-}$and below all the poles of $G_{k \dot{r} \mid \nu}$ which are above this pole of $g_{k \dot{r} \mid \nu}$ but above all the poles of $G_{k \dot{r} \mid \nu}$ which are below this pole of $g_{k \dot{r} \mid \nu}$. If $g_{k \dot{r} \mid \nu}$ has no pole then $C_{k \dot{r}}$ just runs above all the poles of $G_{k \dot{r} \mid \nu}$, see appendix D for explicit examples.

\section{Conclusion}

In this paper, a free field representation for the $\mathrm{ZF}$ algebra of the $\mathrm{SU}(\mathrm{N}) \times \mathrm{SU}(\mathrm{N}) \mathrm{PCF}$ model was found. Similarly to the Fateev-Lashkevich representation [19] for the ZF algebra of the SS model, this representation allows one to construct form factors of $\left(N^{2}-1\right)$-parameter family of exponential fields of the $\mathrm{SU}(\mathrm{N}) \times \mathrm{SU}(\mathrm{N}) \mathrm{PCF}$ model. The precise form of the exponential fields and their relation to the fields which appear in the Lagrangian of the model remain to be determined.

It is also useful to construct the operators $\Lambda(\tilde{O})$ which satisfy the equations 2.19 . They describe in particular the current and energy-momentum tensor operators. The construction of Lukyanov [12] of these operators does not seem to work for the PCF model.

The approach developed should be applicable to any two dimensional relativistic integrable model invariant under a direct sum of two simple Lie or q-deformed algebras. It would be interesting to apply it to the other PCF models [45].

An important problem which can be now addressed is to construct a free field representation for the ZF algebra of the $\mathrm{AdS}_{5} \times \mathrm{S}^{5}$ superstring sigma model in the light-cone gauge. The model is crossing invariant [46] but it is not relativistic invariant which complicates its analytic properties. The most difficult question is to find Green's function $g_{00}$. The results of this paper show that it would satisfy an extra crossing-type equation similar to (5.44), and hopefully one might be able to solve it. Since Green's functions $g_{\mu \nu}$ determine functions $G_{\mu \nu}$ some of which play the role of minimal form factors, it is 
hoped that this approach may shed some light on the $\mathrm{AdS}_{5} \times \mathrm{S}^{5}$ form factors and their analytic properties.

\section{Acknowledgements}

I would like to thank Tristan McLoughlin and Zoltan Bajnok for useful discussions, and the Galileo Galilei Institute for Theoretical Physics (GGI) for the hospitality and INFN for partial support during the completion of this work, within the program "New Developments in AdS3/CFT2 Holography"

\section{A Green's functions derivation}

Green's function $g_{00}(\theta)$ is found from the requirement that it has a simple zero at $\theta=0$ and no poles or zeroes for $\operatorname{Im}(\theta)<0$, and is given by 2.11

$$
g_{00}(\theta)=g_{P C F}(\theta)=\frac{\Gamma\left(\frac{i \theta}{2 \pi}-\frac{1}{N}+1\right) \Gamma\left(\frac{i \theta}{2 \pi}+\frac{1}{N}\right)}{\Gamma\left(\frac{i \theta}{2 \pi}\right) \Gamma\left(\frac{i \theta}{2 \pi}+1\right)}, \quad f_{00}(t)=2 \frac{\sinh \frac{(N-1) \pi t}{N}}{\sinh \pi t} \sinh \frac{\pi t}{N} .
$$

\section{Left-left and right-right Green's functions}

Since up to an overall multiplier the operators $Z_{k \mathrm{i}}$ (and $Z_{1 \dot{k}}$ ) form the ZF algebra of the Gross-Neveu model, the S-matrices $S_{0 \mid k \dot{r}}\left(\theta-\alpha_{k \dot{r}}\right), S_{k \dot{r} \mid l \dot{q}}\left(\alpha_{k \dot{r}}-\alpha_{l \dot{q}}\right)$ and $S_{0 \mid \dot{k} r}\left(\theta-\alpha_{\dot{k}}\right)$, $S_{\dot{k}_{r} \mid \dot{i}_{q}}\left(\alpha_{k_{r}}-\alpha_{i_{q}}\right)$ are the same as the S-matrices $S_{0 k}$ and $S_{k l}$ of the Gross-Neveu model, up to signs and shifts of $\alpha_{k \dot{r}}$. It is easy to determine the most general solution for the S-matrices from the ZF relations (4.3) or (4.4) and show that up to shifts of $\alpha_{k r}$ it is unique. It is convenient to choose the following S-matrices for $S_{0 \mid 1 \dot{r}}, \dot{r}=\dot{1}, \ldots, \dot{N}$

$$
S_{0 \mid 1 \dot{r}}(\theta)=\frac{\theta}{\theta-\frac{2 \pi i}{N}}
$$

for $S_{k \dot{r} \mid k \dot{q}}, k=1, \ldots, N, \dot{r}=\dot{1}, \ldots, \dot{N}$

$$
S_{k \dot{r} \mid k \dot{q}}(\theta)=\frac{\theta-\frac{2 \pi i}{N}}{\theta+\frac{2 \pi i}{N}}
$$

and finally for $S_{k \dot{r} \mid k+1 \dot{q}}, k=1, \ldots, N-1, \dot{r}, \dot{q}=\dot{1}, \ldots, \dot{N}$,

$$
S_{k \dot{r} \mid k+1, \dot{q}}(\theta)=\frac{\theta}{\theta-\frac{2 \pi i}{N}}
$$

and the same expressions for $S_{\mathrm{i}_{r \mid 1} q}$. All the other S-matrices are either related to the listed ones by unitarity or are equal to 1 . This choice leads to a simple pole structure of the left-right Green's functions consistent with the discussion in subsection 3.1. 
The functions $g_{0 \mid 1 \dot{r}}, g_{1 \dot{r} \mid 0}$ and $g_{k \dot{r} \mid k+1, \dot{q}}, g_{k+1, \dot{q} \mid k, \dot{r}}$, however, cannot be the same as $g_{k, k+1}=g_{k+1, k}$ functions for the Gross-Neveu model because it would lead to the appearance of a pole at $\theta_{12}=2 \pi i / N$ in the products $Z_{1 \mathrm{i}}\left(\theta_{1}\right) Z_{2 \dot{1}}\left(\theta_{2}\right)$ and $Z_{1 \mathrm{i}}\left(\theta_{1}\right) Z_{1 \dot{2}}\left(\theta_{2}\right)$, and, say, $Z_{2 \dot{1}}\left(\theta_{1}\right) Z_{3 \dot{1}}\left(\theta_{2}\right)$ and $Z_{1 \dot{2}}\left(\theta_{1}\right) Z_{1 \dot{3}}\left(\theta_{2}\right)$ due to a pole in $g_{k, k+1}$ and $g_{k+1, k}$. Thus, one has to assume that only one of these functions has a pole.

Since $\mathbb{A}_{2 \dot{r}}^{\dagger}(\alpha) \mathbb{A}^{1 \dot{r}}(\alpha) \mathbb{A}_{1 \dot{1}}^{\dagger}(\theta) \sim \delta_{\dot{1} \dot{r}} \delta(\theta-\alpha)$, one expects $g_{0 \mid 1 \dot{1}}(\theta)$ to have a pole at $\theta=0$ while $g_{0 \mid 1 \dot{r}}(\theta), \dot{r} \geq 2$ to be regular at $\theta=0$. Then, the general ansatz for $g_{0 \mid A}$ and $g_{A \mid 0}$ functions is

$$
\begin{aligned}
& g_{0 \mid 1 \mathrm{i}}(\theta)=\frac{\theta-\frac{2 \pi i}{N}}{\theta} h_{0 \mid 1 \mathrm{i}}(\theta), \quad g_{1 \mathrm{i} \mid 0}(\theta)=h_{0 \mid 1 \mathrm{i}}(-\theta), \\
& g_{0 \mid 1 \dot{r}}(\theta)=h_{0 \mid \dot{1}}(\theta), \quad g_{1 \dot{r} \mid 0}(\theta)=\frac{\theta}{\theta+\frac{2 \pi i}{N}} h_{0 \mid \dot{r}}(-\theta), \quad \dot{r} \geq \dot{2}, \\
& g_{0 \mid \mathrm{i} 1}(\theta)=\frac{\theta-\frac{2 \pi i}{N}}{\theta} h_{0 \mid \mathrm{i} 1}(\theta), \quad g_{\mathrm{i} 1 \mid 0}(\theta)=h_{0 \mid \mathrm{i} 1}(-\theta), \\
& g_{0 \mid \mathrm{i} r}(\theta)=h_{0 \mid \mathrm{i} r}(\theta), \quad g_{\mathrm{i} r \mid 0}(\theta)=\frac{\theta}{\theta+\frac{2 \pi i}{N}} h_{0 \mid \mathrm{i} r}(-\theta), \quad r \geq 2,
\end{aligned}
$$

where $h_{0 \mid 1 \dot{r}}(\theta)$ cannot have poles and cannot have a zero at $\theta=0$.

Similarly, the general ansatz for the left-left and right-right Green's functions is

$$
\begin{aligned}
& g_{k \dot{r} \mid k \dot{q}}(\theta)=h_{k \dot{r} \mid k \dot{q}}(\theta), \quad g_{k \dot{q} \mid k \dot{r}}(\theta)=\frac{\theta+\frac{2 \pi i}{N}}{\theta-\frac{2 \pi i}{N}} h_{k \dot{r} \mid k \dot{q}}(-\theta), \quad \dot{r}<\dot{q}, \\
& g_{k \dot{r} \mid k \dot{r}}(\theta)=\frac{\theta}{\theta-\frac{2 \pi i}{N}} h_{k \dot{r} \mid k \dot{r}}(\theta), \quad h_{k \dot{r} \mid k \dot{r}}(-\theta)=h_{k \dot{r} \mid k \dot{r}}(\theta), \\
& g_{\dot{k} \mid \dot{k} q}(\theta)=h_{k_{r} \mid k_{k}}(\theta), \quad g_{\dot{k} \mid \dot{k} r}(\theta)=\frac{\theta+\frac{2 \pi i}{N}}{\theta-\frac{2 \pi i}{N}} h_{k_{r} \mid k_{q}}(-\theta), \quad r<q, \\
& g_{\dot{k} \mid \dot{k} r}(\theta)=\frac{\theta}{\theta-\frac{2 \pi i}{N}} h_{\dot{k}_{r \mid} \mid k_{r}}(\theta), \quad h_{\dot{k}_{r \mid} \mid \dot{k}_{r}}(-\theta)=h_{\dot{k}_{r \mid k} r}(\theta), \\
& g_{k \dot{r} \mid k+1, \dot{q}}(\theta)=\frac{\theta-\frac{2 \pi i}{N}}{\theta} h_{k \dot{r} \mid k+1, \dot{q}}(\theta), \quad g_{k+1, \dot{q} \mid k \dot{r}}(\theta)=h_{k \dot{r} \mid k+1, \dot{q}}(-\theta), \quad \dot{r} \geq \dot{q} \\
& g_{k \dot{r} \mid k+1, \dot{q}}(\theta)=h_{k \dot{r} \mid k+1, \dot{q}}(\theta), \quad g_{k+1, \dot{q} \mid k, \dot{r}}(\theta)=\frac{\theta}{\theta+\frac{2 \pi i}{N}} h_{k \dot{r} \mid k+1, \dot{q}}(-\theta), \quad \dot{r}<\dot{q}, \\
& g_{\dot{k} \mid \dot{k}+\dot{i}, q}(\theta)=\frac{\theta-\frac{2 \pi i}{N}}{\theta} h_{\dot{k} \mid \dot{k}+\dot{1}, q}(\theta), \quad g_{\dot{k}+\dot{1}, q \mid \dot{k} r}(\theta)=h_{\dot{k} \mid \dot{k}+\dot{\mathrm{i}, q}}(-\theta), \quad r \geq q, \\
& g_{\dot{k} \mid \dot{k}+\dot{1}, q}(\theta)=h_{\dot{k}_{r \mid \dot{k}+\dot{1}, q}}(\theta), \quad g_{\dot{k}+\dot{\mathrm{i}}, q \mid \dot{k} r}(\theta)=\frac{\theta}{\theta+\frac{2 \pi i}{N}} h_{\dot{k} \mid \dot{k}+\dot{1}, q}(-\theta), \quad r<q .
\end{aligned}
$$

The analytic properties of the $h$-functions will be discussed later.

All the other Green's functions which are not listed above have no poles. This follows from the commutativity relations discussed in subsection (3.5). They are set to 1. 


\section{Left-right and right-left Green's functions}

The commutativity of left and right algebras charges implies that the corresponding Green's functions satisfy

$$
g_{k \dot{r} \mid \dot{q} n}\left(\alpha_{l}-\beta_{r}\right)=g_{\dot{q} n \mid k \dot{r}}\left(\beta_{r}-\alpha_{l}\right) \quad \Rightarrow \quad S_{k \dot{r} \mid \dot{q} n}\left(\alpha_{l}-\beta_{r}\right)=1 .
$$

Some of these functions have no poles. The simplest choice is to take all nonsingular left-right functions to be equal to 1 . However, according to the discussion in subsection 3.1. the functions (grouped according to the relations below)

$$
\left(g_{k, \dot{n}+\dot{1} \mid \dot{n} k}, g_{k \dot{n} \mid \dot{n}, k+1}\right),
$$

have a pole at $\alpha_{l}=\beta_{r}$

$$
\begin{aligned}
& g_{k, \dot{n}+\mathrm{i} \mid \dot{n} k}(\alpha)=\frac{\alpha+\frac{2 \pi i}{N}}{\alpha} h_{k, \dot{n}+\mathrm{i} \mid \dot{n} k}(\alpha)=g_{\dot{n} k \mid k, \dot{n}+\dot{\mathrm{i}}}(-\alpha), \\
& g_{k \dot{n} \mid \dot{n}, k+1}(\alpha)=\frac{\alpha-\frac{2 \pi i}{N}}{\alpha} h_{k \dot{n} \mid \dot{n}, k+1}(\alpha)=g_{\dot{n}, k+1 \mid k \dot{n}}(-\alpha),
\end{aligned}
$$

where $h$ 's are functions which have no poles, and have no zero at $\alpha=0$. It is easy to show that the commutativity of left and right algebras charges then leads to the relations of the form

$$
\begin{aligned}
\phi_{k, \dot{n}+\dot{1}}(\alpha)+\dot{\phi}_{\dot{n} k}(\alpha) & =\phi_{k \dot{n}}(\alpha)+\dot{\phi}_{\dot{n}, k+1}(\alpha), \\
c_{k, \dot{n}+1}(\alpha) c_{\dot{n} k}(\alpha) r_{\dot{n} k \mid k, \dot{n}+1} & =c_{k \dot{n}}(\alpha) c_{\dot{n}, k+1}(\alpha) r_{k \dot{n} \mid \dot{n}, k+1}, \quad 1 \leq k \leq N-1, \quad \dot{1} \leq \dot{n} \leq \dot{N}-\dot{1},
\end{aligned}
$$

where $\left.r_{k \dot{n} \mid \dot{n}, k+1} \equiv \operatorname{Res}\left(g_{k \dot{n} \mid \dot{n}, k+1}(\alpha)\right)\right|_{\alpha=0},\left.r_{\dot{n} k \mid k, \dot{n}+1} \equiv \operatorname{Res}\left(g_{\dot{n} k \mid k, \dot{n}+1}(\alpha)\right)\right|_{\alpha=0}$.

These relations lead to a huge number of the following additional relations between various Green's functions

$$
\begin{aligned}
& g_{A \mid k, \dot{n}+\dot{1}}(\alpha) g_{A \mid \dot{n} k}(\alpha)=g_{A \mid k \dot{n}}(\alpha) g_{A \mid \dot{n}, k+1}(\alpha), \\
& g_{k, \dot{n}+\dot{1} \mid A}(\alpha) g_{\dot{n} k \mid A}(\alpha)=g_{k \dot{n} \mid A}(\alpha) g_{\dot{n}, k+1 \mid A}(\alpha),
\end{aligned}
$$

where $A$ is any of the indices of the fields. Taking the ratio of these equations, one gets the following relations between the S-matrices

$$
S_{A \mid k, \dot{n}+\mathrm{i}}(\alpha) S_{A \mid \dot{n} k}(\alpha)=S_{A \mid k \dot{n}}(\alpha) S_{A \mid \dot{n}, k+1}(\alpha),
$$

which are indeed satisfied. Thus, it is sufficient to consider just one set of these equations.

Now, taking $A=0$, one gets

$$
g_{0 \mid k, \dot{n}+\dot{1}}(\alpha) g_{0 \mid \dot{n} k}(\alpha)=g_{0 \mid k \dot{n}}(\alpha) g_{0 \mid \dot{n}, k+1}(\alpha) .
$$

Nontrivial relations can appear only for $k=1$ or $\dot{n}=1$. One gets for $k=1$ and $\dot{n} \geq \dot{2}$

$$
g_{0 \mid 1, \dot{n}+\dot{1}}(\alpha)=g_{0 \mid 1 \dot{n}}(\alpha) \Rightarrow h_{0 \mid 1 \dot{n}}(\alpha)=h_{0 \mid 1 \dot{2}}(\alpha), \quad \dot{n} \geq \dot{2} .
$$


Similarly, for $k \geq 2$ and $\dot{n}=\dot{1}$

$$
g_{0 \mid \mathrm{i} k}(\alpha)=g_{0 \mid \mathrm{i}, k+1}(\alpha) \Rightarrow h_{0 \mid \mathrm{i} k}(\alpha)=h_{0 \mid \mathrm{i} 2}(\alpha), \quad k \geq 2 .
$$

Finally, for $k=1$ and $\dot{n}=1$

$$
g_{0 \mid 1 \dot{2}}(\alpha) g_{0 \mid \mathrm{i} 1}(\alpha)=g_{0 \mid 1 \mathrm{i}}(\alpha) g_{0 \mid \mathrm{i} 2}(\alpha) \Rightarrow h_{0 \mid 1 \dot{2}}(\alpha) h_{0 \mid \mathrm{i} 1}(\alpha)=h_{0 \mid 1 \mathrm{i}}(\alpha) h_{0 \mid \mathrm{i} 2}(\alpha),
$$

and therefore there are three independent functions $h_{0 \mid A}$.

Next, taking $A=m \dot{r}$, one gets the following relations between Green's functions

$$
g_{m \dot{r} \mid k, \dot{n}+\dot{1}}(\alpha) g_{m \dot{r} \mid \dot{n} k}(\alpha)=g_{m \dot{r} \mid k \dot{n}}(\alpha) g_{m \dot{r} \mid \dot{n}, k+1}(\alpha),
$$

There are several cases to be considered.

1. $m \leq k-2$ or $m \geq k+2$

The equations are satisfied because all these Green's functions are equal to 1.

2. $m=k-1: \quad g_{k-1, \dot{r} \mid k, \dot{n}+\dot{1}}(\alpha) g_{k-1, \dot{r} \mid \dot{n} k}(\alpha)=g_{k-1, \dot{r} \mid k \dot{n}}(\alpha)$. Then,

$$
\begin{array}{r}
g_{k-1, \dot{n} \mid k, \dot{n}+\mathrm{i}}(\alpha) g_{k-1, \dot{n} \mid \dot{n} k}(\alpha)=g_{k-1, \dot{n} \mid k \dot{n}}(\alpha) \Rightarrow h_{k-1, \dot{n} \mid k, \dot{n}+\mathrm{i}}(\alpha) h_{k-1, \dot{n} \mid \dot{n} k}(\alpha)=h_{k-1, \dot{n} \mid k \dot{n}}(\alpha), \\
g_{k-1, \dot{r} \mid k, \dot{n}+\mathrm{i}}(\alpha)=g_{k-1, \dot{r} \mid k \dot{n}}(\alpha) \Rightarrow h_{k-1, \dot{r} \mid k, \dot{n}+\mathrm{i}}(\alpha)=h_{k-1, \dot{r} \mid k \dot{n}}(\alpha) \quad \text { if } \quad \dot{r} \neq \dot{n} .
\end{array}
$$

3. $m=k+1: \quad g_{k+1, \dot{r} \mid k, \dot{n}+\dot{1}}(\alpha)=g_{k+1, \dot{r} \mid k \dot{n}}(\alpha) g_{k+1, \dot{r} \mid \dot{n}, k+1}(\alpha)$. Then,

$$
\begin{gathered}
g_{k+1, \dot{n}+\mathrm{i} \mid k, \dot{n}+\mathrm{i}}(\alpha)=g_{k+1, \dot{n}+\mathrm{i} \mid k \dot{n}}(\alpha) g_{k+1, \dot{n}+\mathrm{i} \mid \dot{n}, k+1}(\alpha) \Rightarrow \\
h_{k, \dot{n}+\mathrm{i} \mid k+1, \dot{n}+\mathrm{i}}(\alpha)=h_{k \dot{n} \mid k+1, \dot{n}+\mathrm{i}}(\alpha) h_{k+1, \dot{n}+\mathrm{i} \mid \dot{n}, k+1}(-\alpha) . \\
g_{k+1, \dot{r} \mid k, \dot{n}+\mathrm{i}}(\alpha)=g_{k+1, \dot{r} \mid k \dot{n}}(\alpha) \Rightarrow h_{k, \dot{n}+\dot{\mathrm{i}} \mid k+1, \dot{r}}(\alpha)=h_{k \dot{n} \mid k+1, \dot{r}}(\alpha) \quad \text { if } \quad \dot{r} \neq \dot{n}+\dot{1} .
\end{gathered}
$$

Thus

$$
\begin{aligned}
h_{k \dot{n} \mid k+1, \dot{r}}(\alpha) & =h_{k \dot{n} \mid k+1, \mathrm{i}}(\alpha)=h_{k \mathrm{i} \mid k+1, \mathrm{i}}(\alpha), \quad \dot{n} \geq \dot{r}, \\
h_{k \dot{n} \mid k+1, \dot{r}}(\alpha) & =h_{k \mathrm{i} \mid k+1, \dot{r}}(\alpha)=h_{k \mathrm{i} \mid k+1, \dot{2}}(\alpha), \quad \dot{n}<\dot{r}, \\
h_{k \dot{n} \mid \dot{n}, k+1}(\alpha) & =h_{k \dot{\mathrm{i} \mid \mathrm{i}, k+1}}(\alpha), \\
h_{k, \dot{n}+1 \mid \dot{n} k}(\alpha) & =h_{k \dot{2} \mid \mathrm{i} k}(\alpha), \\
h_{k \mathrm{i} \mid k+1, \dot{\mathrm{i}}}(\alpha) & =h_{k \mathrm{i} \mid k+1, \dot{2}}(\alpha) h_{k+1, \dot{2} \mid \mathrm{i}, k+1}(-\alpha), \\
h_{k \dot{\mathrm{i}} \mid k+1, \mathrm{i}}(\alpha) & =h_{k \dot{\mathrm{i}} \mid k+1, \dot{2}}(\alpha) h_{k \dot{\mathrm{i}} \mid \mathrm{i}, k+1}(\alpha), \\
h_{k+1, \dot{2} \mid \mathrm{i}, k+1}(-\alpha) & =h_{k \mathrm{i} \mid \mathrm{i}, k+1}(\alpha) .
\end{aligned}
$$

4. $m=k: \quad g_{k \dot{r} \mid k, \dot{n}+\dot{1}}(\alpha) g_{k \dot{r} \mid \dot{n} k}(\alpha)=g_{k \dot{r} \mid k \dot{n}}(\alpha) g_{k \dot{r} \mid \dot{n}, k+1}(\alpha)$. Then,

$$
g_{k \dot{n} \mid k, \dot{n}+\dot{1}}(\alpha)=g_{k \dot{n} \mid k \dot{n}}(\alpha) g_{k \dot{n} \mid \dot{n}, k+1}(\alpha) \Rightarrow h_{k \dot{n} \mid k, \dot{n}+\dot{1}}(\alpha)=h_{k \dot{n} \mid k \dot{n}}(\alpha) h_{k \dot{n} \mid \dot{n}, k+1}(\alpha) \text {, }
$$




$$
\begin{gathered}
g_{k, \dot{n}+\dot{\mathrm{i}} \mid k, \dot{n}+\mathrm{i}}(\alpha) g_{k, \dot{n}+\mathrm{i} \mid \dot{n} k}(\alpha)=g_{k, \dot{n}+\dot{\mathrm{i}} \mid k \dot{n}}(\alpha) \Rightarrow h_{k, \dot{n}+\mathrm{i} \mid k, \dot{n}+\mathrm{i}}(\alpha) h_{k, \dot{n}+\mathrm{i} \mid \dot{n} k}(\alpha)=h_{k, \dot{n}+\dot{\mathrm{i}} \mid k \dot{n}}(\alpha) \\
g_{k \dot{r} \mid k, \dot{n}+\mathrm{i}}(\alpha)=g_{k \dot{r} \mid k \dot{n}}(\alpha) \Rightarrow h_{k \dot{r} \mid k, \dot{n}+\mathrm{i}}(\alpha)=h_{k \dot{r} \mid k \dot{n}}(\alpha) \quad \text { if } \quad \dot{r} \neq \dot{n} \text { or } \dot{r} \neq \dot{n}+\dot{1}
\end{gathered}
$$

Thus,

$$
\begin{aligned}
h_{k \dot{n} \mid k \dot{r}}(\alpha) & =h_{k \mathrm{i} \mid k \dot{2}}(\alpha), \quad \dot{n}<\dot{r}, \\
h_{k \dot{n} \mid k \dot{n}}(\alpha) & =h_{k \dot{\mathrm{i} \mid k \mathrm{i}}}(\alpha), \\
h_{k \mathrm{i} \mid k \dot{2}}(\alpha) & =h_{k \mathrm{i} \mid k \mathrm{i}}(\alpha) h_{k \dot{\mathrm{i} \mid \mathrm{i}, k+1}}(\alpha), \\
h_{k \dot{\mathrm{i}} \mid k \dot{2}}(-\alpha) & =h_{k \mathrm{i} \mid k \mathrm{i}}(\alpha) h_{k \dot{2} \mid \mathrm{i} k}(\alpha), \\
h_{k \dot{2} \mid \mathrm{i} k}(-\alpha) & =h_{k \dot{\mathrm{i} \mid \mathrm{i}, k+1}}(\alpha)=h_{k+1, \dot{2} \mid \mathrm{i}, k+1}(-\alpha)=h_{k-1, \dot{\mathrm{i}} \mid \mathrm{i}, k}(\alpha)=h_{1 \mathrm{i} \mid \mathrm{i} 2}(\alpha) .
\end{aligned}
$$

The functions $h_{\dot{n} k \mid \dot{r} q}$ satisfy similar relations. The simplest solution used in the paper is obviously $h_{A \mid B}=1$ for any $A, B$.

\section{B Constraints and elementary free fields}

\section{Solving the constraints}

The constraints

$$
\phi_{k \dot{r}}(\theta)+\dot{\phi}_{\dot{r}, k+1}(\theta)=\dot{\phi}_{\dot{r} k}(\theta)+\phi_{k, \dot{r}+\dot{1}}(\theta)
$$

between the fields can be easily solved. In terms of the new fields

$$
\begin{aligned}
& \varphi_{k \dot{1}}=\phi_{k \dot{1}}, \varphi_{k \dot{2}}=\phi_{k \dot{2}}-\phi_{k \dot{1}}, \varphi_{k \dot{3}}=\phi_{k \dot{3}}-\phi_{k \dot{2}}, \ldots, \varphi_{k \dot{N}}=\phi_{k \dot{N}}-\phi_{k, \dot{N}-\dot{1}} \\
& \varphi_{k \dot{r}}=\phi_{k \dot{r}}-\phi_{k, \dot{r}-\dot{1}}, \quad \phi_{k \dot{0}}=0, \quad k=1, \ldots, N-1, \quad \dot{r}=\dot{1}, \ldots, \dot{N}, \\
& \phi_{k \dot{1}}=\varphi_{k \dot{1}}, \phi_{k \dot{2}}=\varphi_{k \dot{2}}+\varphi_{k \dot{1}}, \phi_{k \dot{3}}=\varphi_{k \dot{3}}+\varphi_{k \dot{2}}+\varphi_{k \dot{1}}, \ldots, \\
& \phi_{k \dot{r}}=\sum_{\dot{q}=\dot{1}}^{\dot{r}} \varphi_{k \dot{q}}, \quad k=1, \ldots, N-1, \quad \dot{r}=\dot{1}, \ldots, \dot{N}, \\
& \dot{\varphi}_{k 1}=\dot{\phi}_{k 1}, \dot{\varphi}_{k 2}=\dot{\phi}_{\dot{k} 2}-\dot{\phi}_{k 1}, \dot{\varphi}_{k 3}=\dot{\phi}_{\dot{k} 3}-\dot{\phi}_{\dot{k} 2}, \ldots, \dot{\varphi}_{k_{N}}=\dot{\phi}_{k_{N}}-\dot{\phi}_{k, N-1}, \\
& \dot{\varphi}_{k r}=\dot{\phi}_{k r}-\dot{\phi}_{\dot{k}, r-1}, \quad \dot{\phi}_{k 0}=0, \quad \dot{k}=\dot{1}, \ldots, \dot{N}-\dot{1}, \quad r=1, \ldots, N, \\
& \dot{\phi}_{k 1}=\dot{\varphi}_{\dot{k} 1}, \dot{\phi}_{k 2}=\dot{\varphi}_{k 2}+\dot{\varphi}_{\dot{k} 1}, \dot{\phi}_{\dot{k} 3}=\dot{\varphi}_{\dot{k} 3}+\dot{\varphi}_{\dot{k} 2}+\dot{\varphi}_{\dot{k} 1}, \ldots, \\
& \dot{\phi}_{k r}=\sum_{q=1}^{r} \dot{\varphi}_{k q}, \quad \dot{k}=\dot{1}, \ldots, \dot{N}-\dot{1}, \quad r=1, \ldots, N,
\end{aligned}
$$

the constraints take the form

$$
\varphi_{k, \dot{r}+\dot{1}}=\dot{\varphi}_{\dot{r}, k+1} \equiv \psi_{k r}, \quad k=1, \ldots, N-1, \quad r=1, \ldots, N-1,
$$


and the solution is written in terms of $\phi_{k \dot{1}}, \dot{\phi}_{\dot{k} 1}$, and $\psi_{k r}$

$$
\phi_{k \dot{r}}=\phi_{k \dot{1}}+\sum_{q=1}^{r-1} \psi_{k q}=\sum_{q=0}^{r-1} \psi_{k q}, \quad \dot{\phi}_{k r}=\dot{\phi}_{k 1}+\sum_{q=1}^{r-1} \psi_{q k}=\sum_{q=0}^{r-1} \psi_{q k},
$$

where

$$
\psi_{k 0} \equiv \phi_{k \dot{1}}, \quad \psi_{0 k} \equiv \dot{\phi}_{\dot{k} 1} .
$$

Green's functions of $\psi_{k q}$ 's are given by

$$
\begin{gathered}
g_{k r \mid n q}^{\psi \psi}(\theta)=\frac{g_{k, \dot{r}+\dot{\mathrm{i}} \mid n, \dot{q}+\dot{\mathrm{q}}}(\theta) g_{k \dot{r} \mid n \dot{q}}(\theta)}{g_{k, \dot{r}+\dot{\mathrm{i}} \mid n \dot{q}}(\theta) g_{k \dot{r} \mid n, \dot{q}+\dot{\mathrm{i}}}(\theta)}, \quad k, n \geq 1, \\
g_{0 r \mid n q}^{\psi \psi}(\theta)=g_{\dot{r} 1 \mid n q}^{\phi \psi}(\theta)=\frac{g_{\dot{r} 1 \mid n, \dot{q}+\dot{\mathrm{i}}}(\theta)}{g_{\dot{r} 1 \mid n \dot{q}}(\theta)}, \quad n \geq 1, \\
g_{k r \mid 0 q}^{\psi \psi}(\theta)=g_{k r \mid \dot{q} 1}^{\psi \phi}(\theta)=\frac{g_{k, \dot{r}+\dot{\mathrm{i}} \mid \dot{q} 1}(\theta)}{g_{k \dot{r} \mid \dot{q} 1}(\theta)}, \quad k \geq 1, \\
g_{0 r \mid 0 q}^{\psi \psi}(\theta)=g_{\dot{r} 1 \mid \dot{q} 1}(\theta) .
\end{gathered}
$$

The functions different from 1 are

$$
\begin{aligned}
& g_{k r \mid k+1, r+1}^{\psi \psi}(\theta)=\frac{g_{k, \dot{r}+\mathrm{i} \mid k+1, \dot{r}+\dot{2}}(\theta) g_{k \dot{r} \mid k+1, \dot{r}+\dot{1}}(\theta)}{g_{k, \dot{r}+\mathrm{i} \mid k+1, \dot{r}+\dot{\mathrm{i}}}(\theta) g_{k \dot{r} \mid k+1, \dot{r}+\dot{2}}(\theta)}=\frac{\theta}{\theta-\frac{2 \pi i}{N}}, \quad k \geq 1, \dot{r} \geq \dot{0}, \\
& g_{k+1, r+1 \mid k r}^{\psi \psi}(\theta)=\frac{g_{k+1, \dot{r}+\dot{2} \mid k, \dot{r}+\dot{1}}(\theta) g_{k+1, \dot{r}+\dot{i} \mid k \dot{r}}(\theta)}{g_{k+1, \dot{r}+\dot{2} \mid k, \dot{r}}(\theta) g_{k+1, \dot{r}+1 \mid k, \dot{r}+1}(\theta)}=\frac{\theta}{\theta+\frac{2 \pi i}{N}}, \quad k \geq 1, \dot{r} \geq \dot{0}, \\
& g_{k r \mid k+1, r}^{\psi \psi}(\theta)=\frac{g_{k, \dot{r}+\mathrm{i} \mid k+1, \dot{r}+\dot{\mathrm{i}}}(\theta) g_{k \dot{r} \mid k+1, \dot{r}}(\theta)}{g_{k, \dot{r}+\mathrm{i} \mid k+1, \dot{r}}(\theta) g_{k \dot{r} \mid k+1, \dot{r}+\dot{\mathrm{i}}}(\theta)}=\frac{\theta-\frac{2 \pi i}{N}}{\theta}, \quad k \geq 1, \dot{r} \geq \dot{0}, \\
& g_{k+1, r \mid k r}^{\psi \psi}(\theta)=\frac{g_{k+1, \dot{r}+\dot{\mathrm{i}} \mid k, \dot{r}+\dot{\mathrm{i}}}(\theta) g_{k+1, \dot{r} \mid k \dot{r}}(\theta)}{g_{k+1, \dot{r}+\dot{\mathrm{i}} \mid k \dot{r}}(\theta) g_{k+1, \dot{r} \mid k, \dot{r}+\mathrm{i}}(\theta)}=\frac{\theta+\frac{2 \pi i}{N}}{\theta}, \quad k \geq 1, \dot{r} \geq \dot{1}, \\
& g_{k r \mid k, r+1}^{\psi \psi}(\theta)=\frac{g_{k, \dot{r}+\dot{\mathrm{i}} \mid k, \dot{r}+\dot{2}}(\theta) g_{k \dot{r} \mid k, \dot{r}+1}(\theta)}{g_{k, \dot{r}+\mathrm{i} \mid k, \dot{r}+1}(\theta) g_{k \dot{r} \mid k, \dot{r}+\dot{2}}(\theta)}=\frac{\theta-\frac{2 \pi i}{N}}{\theta}, \quad k \geq 1, \dot{r} \geq \dot{0} \\
& g_{k, r+1 \mid k \dot{r}}^{\psi \psi}(\theta)=\frac{g_{k, \dot{r}+\dot{2} \mid k, \dot{r}}(\theta) g_{k, \dot{r}+\dot{\mathrm{i}} \mid k \dot{r}}(\theta)}{g_{k, \dot{r}+\dot{2} \mid k \dot{r}}(\theta) g_{k, \dot{r}+\dot{1} \mid k, \dot{r}+\dot{1}}(\theta)}=\frac{\theta+\frac{2 \pi i}{N}}{\theta}, \quad k \geq 1, \dot{r} \geq \dot{0}, \\
& g_{k r \mid k r}^{\psi \psi}(\theta)=\frac{g_{k, \dot{r}+\dot{\mathrm{i}} \mid k, \dot{r}+\mathrm{i}}(\theta) g_{k \dot{r} \mid k \dot{r}}(\theta)}{g_{k, \dot{r}+\mathrm{i} \mid k \dot{r}}(\theta) g_{k \dot{r} \mid k, \dot{r}+\dot{1}}(\theta)}=\frac{\theta^{2}}{\theta^{2}+\frac{4 \pi^{2}}{N^{2}}}, \quad k \geq 1, \dot{r} \geq \dot{1}, \\
& g_{k 0 \mid k 0}^{\psi \psi}(\theta)=g_{k \mathrm{i} \mid k \mathrm{i}}(\theta)=\frac{\theta}{\theta-\frac{2 \pi i}{N}}, \quad k \geq 1, \\
& g_{0 r \mid 1 r}^{\psi \psi}(\theta)=g_{\dot{r} 1 \mid 1 r}^{\phi \psi}(\theta)=\frac{g_{\dot{r} 1 \mid 1, \dot{r}+\dot{1}}(\theta)}{g_{\dot{r} 1 \mid 1 \dot{r}}(\theta)}=\frac{\theta-\frac{2 \pi i}{N}}{\theta}, \quad \dot{r} \geq \dot{1},
\end{aligned}
$$




$$
\begin{gathered}
g_{1 r \mid 0 r}^{\psi \psi}(\theta)=g_{1 r \mid \dot{r} 1}^{\psi \phi}(\theta)=\frac{g_{1, \dot{r}+\dot{1} \mid \dot{r} 1}(\theta)}{g_{1 \dot{r} \mid \dot{r} 1}(\theta)}=\frac{\theta+\frac{2 \pi i}{N}}{\theta}, \quad \dot{r} \geq \dot{1}, \\
g_{0 r \mid 1, r+1}^{\psi \psi}(\theta)=g_{\dot{r} 1 \mid 1, r+1}^{\phi \psi}(\theta)=\frac{g_{\dot{r} 1 \mid 1, \dot{r}+\dot{2}}(\theta)}{g_{\dot{r} 1 \mid 1, \dot{r}+\dot{1}}(\theta)}=\frac{\theta}{\theta-\frac{2 \pi i}{N}}, \quad \dot{r} \geq \dot{1}, \\
g_{1, r+1 \mid 0 r}^{\psi \psi}(\theta)=g_{1, r+1 \mid \dot{r} 1}^{\psi \phi}(\theta)=\frac{g_{1, \dot{r}+\dot{2} \mid \dot{r} 1}(\theta)}{g_{1, \dot{r}+\dot{1} \mid \dot{r} 1}(\theta)}=\frac{\theta}{\theta+\frac{2 \pi i}{N}}, \quad \dot{r} \geq \dot{1}, \\
g_{0 r \mid 0, r+1}^{\psi \psi}(\theta)=g_{\dot{r} 1 \mid \dot{r}+\dot{1}, 1}(\theta)=\frac{\theta-\frac{2 \pi i}{N}}{\theta}, \quad \dot{r} \geq \dot{1}, \\
g_{0 r \mid 0 r}^{\psi \psi}(\theta)=g_{\dot{r} 1 \mid \dot{r} 1}(\theta)=\frac{\theta}{\theta-\frac{2 \pi i}{N}}, \quad \dot{r} \geq \dot{1} .
\end{gathered}
$$

It is interesting that the only Green's functions with S-matrices different from 1 are $g_{0 r \mid 0, r+1}^{\psi \psi}, g_{0 r \mid 0 r}^{\psi \psi}, g_{k 0 \mid k+1,0}^{\psi \psi}, g_{k 0 \mid k 0}^{\psi \psi}$.

\section{Elementary free fields}

It seems that the best way to handle the free fields is to introduce $N^{2}-1$ elementary fields $\xi_{k r}(\theta), k, r=0,1, \ldots N-1, \xi_{00}(\theta)=0$, which satisfy the simplest commutation relations

$$
\begin{gathered}
\xi_{k r}(\theta)=\int_{-\infty}^{\infty} \frac{d t}{i t} a_{k r}(t) e^{i \theta t}=\xi_{k r}^{-}(\theta)+\xi_{k r}^{+}(\theta), \quad\left[a_{k r}(t), a_{n q}\left(t^{\prime}\right)\right]=t \delta_{k r, n q} \delta\left(t+t^{\prime}\right), \\
\left\langle\xi_{n q}\left(\theta_{1}\right) \xi_{k r}\left(\theta_{2}\right)\right\rangle=-\log g_{k r \mid n q}\left(\theta_{21}\right)=-\delta_{k r, n q} \log i e^{\gamma} \theta_{21}, \quad g_{k r \mid k r}(\theta)=i e^{\gamma} \theta
\end{gathered}
$$

where $\gamma$ is Euler's constant.

There are infinitely many different ways to represent $\psi_{k r}$ in terms of the elementary fields. By using the ansatz

$$
\begin{aligned}
& \psi_{k r}^{+}(\theta)=\xi_{k r}^{+}(\theta), \\
& \psi_{k r}^{-}(\theta)=\sum_{A}\left(d_{k r \mid A}\left(\xi_{A}^{-}\left(\theta-\mathfrak{u}_{2}\right)-\xi_{A}^{-}(\theta)\right)+f_{k r \mid A}\left(\xi_{A}^{-}\left(\theta+\mathfrak{u}_{2}\right)-\xi_{A}^{-}(\theta)\right)\right),
\end{aligned}
$$

where $\xi_{A}$ are the $N^{2}-1$ elementary fields, one finds the following representation

$$
\begin{aligned}
\psi_{k r}^{-}(\theta) & =2 \xi_{k r}^{-}(\theta)-\xi_{k r}^{-}\left(\theta-\mathfrak{u}_{2}\right)-\xi_{k r}^{-}\left(\theta+\mathfrak{u}_{2}\right) \\
& +\xi_{k-1, r-1}^{-}(\theta)-\xi_{k-1, r-1}^{-}\left(\theta+\mathfrak{u}_{2}\right)+\xi_{k+1, r+1}^{-}(\theta)-\xi_{k+1, r+1}^{-}\left(\theta-\mathfrak{u}_{2}\right) \\
& -\xi_{k+1, r}^{-}(\theta)+\xi_{k+1, r}^{-}\left(\theta-\mathfrak{u}_{2}\right)-\xi_{k-1, r}^{-}(\theta)+\xi_{k-1, r}^{-}\left(\theta+\mathfrak{u}_{2}\right) \\
& -\xi_{k, r+1}^{-}(\theta)+\xi_{k, r+1}^{-}\left(\theta-\mathfrak{u}_{2}\right)-\xi_{k, r-1}^{-}(\theta)+\xi_{k, r-1}^{-}\left(\theta+\mathfrak{u}_{2}\right) \\
\psi_{k r}^{+}(\theta) & =\xi_{k r}^{+}(\theta), \quad k \geq 2, r \geq 1 \\
\psi_{k 0}^{-}(\theta) & =\xi_{k 0}^{-}(\theta)-\xi_{k 0}^{-}\left(\theta+\mathfrak{u}_{2}\right)-\xi_{k 1}^{-}(\theta)+\xi_{k 1}^{-}\left(\theta-\mathfrak{u}_{2}\right) \\
& +\xi_{k+1,1}^{-}(\theta)-\xi_{k+1,1}^{-}\left(\theta-\mathfrak{u}_{2}\right)-\xi_{k-1,0}^{-}(\theta)+\xi_{k-1,0}^{-}\left(\theta+\mathfrak{u}_{2}\right) \\
\psi_{k 0}^{+}(\theta) & =\xi_{k 0}^{+}(\theta), \quad k \geq 2
\end{aligned}
$$




$$
\begin{aligned}
\psi_{10}^{-}(\theta) & =\xi_{10}^{-}(\theta)-\xi_{10}^{-}\left(\theta+\mathfrak{u}_{2}\right)-\xi_{11}^{-}(\theta)+\xi_{11}^{-}\left(\theta-\mathfrak{u}_{2}\right) \\
& +\xi_{21}^{-}(\theta)-\xi_{21}^{-}\left(\theta-\mathfrak{u}_{2}\right) \\
\psi_{10}^{+}(\theta) & =\xi_{10}^{+}(\theta) \\
\psi_{1 r}^{-}(\theta) & =2 \xi_{1 r}^{-}(\theta)-\xi_{1 r}^{-}\left(\theta-\mathfrak{u}_{2}\right)-\xi_{1 r}^{-}\left(\theta+\mathfrak{u}_{2}\right) \\
& +\xi_{0, r-1}^{-}(\theta)-\xi_{0, r-1}^{-}\left(\theta+\mathfrak{u}_{2}\right)+\xi_{2, r+1}^{-}(\theta)-\xi_{2, r+1}^{-}\left(\theta-\mathfrak{u}_{2}\right) \\
& -\xi_{2 r}^{-}(\theta)+\xi_{2 r}^{-}\left(\theta-\mathfrak{u}_{2}\right)-\xi_{0 r}^{-}(\theta)+\xi_{0 r}^{-}\left(\theta+\mathfrak{u}_{2}\right) \\
& -\xi_{1, r+1}^{-}(\theta)+\xi_{1, r+1}^{-}\left(\theta-\mathfrak{u}_{2}\right)-\xi_{1, r-1}^{-}(\theta)+\xi_{1, r-1}^{-}\left(\theta+\mathfrak{u}_{2}\right), \\
\psi_{1 r}^{+}(\theta) & =\xi_{1 r}^{+}(\theta), \quad r \geq 2, \\
\psi_{11}^{-}(\theta) & =2 \xi_{11}^{-}(\theta)-\xi_{11}^{-}\left(\theta-\mathfrak{u}_{2}\right)-\xi_{11}^{-}\left(\theta+\mathfrak{u}_{2}\right)+\xi_{22}^{-}(\theta)-\xi_{22}^{-}\left(\theta-\mathfrak{u}_{2}\right) \\
& -\xi_{21}^{-}(\theta)+\xi_{21}^{-}\left(\theta-\mathfrak{u}_{2}\right)-\xi_{01}^{-}(\theta)+\xi_{01}^{-}\left(\theta+\mathfrak{u}_{2}\right) \\
& -\xi_{12}^{-}(\theta)+\xi_{12}^{-}\left(\theta-\mathfrak{u}_{2}\right)-\xi_{10}^{-}(\theta)+\xi_{10}^{-}\left(\theta+\mathfrak{u}_{2}\right) \\
\psi_{11}^{+}(\theta) & =\xi_{11}^{+}(\theta), \quad \\
\psi_{0 r}^{-}(\theta) & =\xi_{0 r}^{-}(\theta)-\xi_{0 r}^{-}\left(\theta+\mathfrak{u}_{2}\right)+\xi_{1, r+1}^{-}(\theta)-\xi_{1, r+1}^{-}\left(\theta-\mathfrak{u}_{2}\right) \\
& -\xi_{1 r}^{-}(\theta)+\xi_{1 r}^{-}\left(\theta-\mathfrak{u}_{2}\right)-\xi_{0, r-1}^{-}(\theta)+\xi_{0, r-1}^{-}\left(\theta+\mathfrak{u}_{2}\right) \\
\psi_{0 r}^{+}(\theta) & =\xi_{0 r}^{+}(\theta), \quad r \geq 2 \\
\psi_{01}^{-}(\theta) & =\xi_{01}^{-}(\theta)-\xi_{01}^{-}\left(\theta+\mathfrak{u}_{2}\right)+\xi_{12}^{-}(\theta)-\xi_{12}^{-}\left(\theta-\mathfrak{u}_{2}\right) \\
& -\xi_{11}^{-}(\theta)+\xi_{11}^{-}\left(\theta-\mathfrak{u}_{2}\right) \\
\psi_{01}^{+}(\theta) & =\xi_{01}^{+}(\theta)
\end{aligned}
$$

These formulae are used to express $\phi_{k \dot{r}}$ and $\phi_{k r}$ in terms of the elementary fields

$$
\begin{aligned}
\phi_{k \dot{r}}^{-}(\theta) & =\xi_{k, r-1}^{-}(\theta)-\xi_{k, r-1}^{-}\left(\theta+\mathfrak{u}_{2}\right)-\xi_{k-1, r-1}^{-}(\theta)+\xi_{k-1, r-1}^{-}\left(\theta+\mathfrak{u}_{2}\right) \\
& +\xi_{k+1, r}^{-}(\theta)-\xi_{k+1, r}^{-}\left(\theta-\mathfrak{u}_{2}\right)-\xi_{k r}^{-}(\theta)+\xi_{k r}^{-}\left(\theta-\mathfrak{u}_{2}\right), \\
\dot{\phi}_{\dot{k} r}^{-}(\theta) & =\xi_{r-1, k}^{-}(\theta)-\xi_{r-1, k}^{-}\left(\theta+\mathfrak{u}_{2}\right)-\xi_{r-1, k-1}^{-}(\theta)+\xi_{r-1, k-1}^{-}\left(\theta+\mathfrak{u}_{2}\right) \\
& -\xi_{r k}^{-}(\theta)+\xi_{r k}^{-}\left(\theta-\mathfrak{u}_{2}\right)+\xi_{r, k+1}^{-}(\theta)-\xi_{r, k+1}^{-}\left(\theta-\mathfrak{u}_{2}\right), \\
\phi_{k \dot{r}}^{+}(\theta) & =\sum_{q=0}^{r-1} \xi_{k q}^{+}(\theta), \quad \dot{\phi}_{\dot{k} r}^{+}(\theta)=\sum_{q=0}^{r-1} \xi_{q k}^{+}(\theta),
\end{aligned}
$$

and therefore (summing over $m, n$ )

$$
\begin{aligned}
\phi_{k \dot{r}}^{-}(\theta) & =\int_{0}^{\infty} \frac{d t}{i t} \Phi_{k \dot{r}, m n}^{-}(t) a_{m n}(t) e^{i \theta t}, \\
\Phi_{k \dot{r}, m n}^{-}(t) & =\left(1-e^{-\frac{2 \pi}{N} t}\right)\left(\delta_{k m} \delta_{r-1, n}-\delta_{k-1, m} \delta_{r-1, n}\right)+\left(1-e^{\frac{2 \pi}{N} t}\right)\left(\delta_{k+1, m} \delta_{r n}-\delta_{k m} \delta_{r n}\right), \\
\dot{\phi}_{\dot{k} r}^{-}(\theta) & =\int_{0}^{\infty} \frac{d t}{i t} \Phi_{\dot{k} r, m n}^{-}(t) a_{m n}(t) e^{i \theta t}, \quad \Phi_{\dot{k} r, m n}^{-}(t)=\Phi_{k \dot{r}, n m}^{-}(t),
\end{aligned}
$$




$$
\begin{aligned}
\phi_{k \dot{r}}^{+}(\theta) & =\int_{-\infty}^{0} \frac{d t}{i t} \Phi_{k \dot{r}, m n}^{+}(t) a_{m n}(t) e^{i \theta t}, & \Phi_{k \dot{r}, m n}^{+}(t) & =\delta_{k m}(1-u(n-r)), \\
\phi_{\dot{k} r}^{+}(\theta) & =\int_{-\infty}^{0} \frac{d t}{i t} \Phi_{\dot{k} r, m n}^{+}(t) a_{m n}(t) e^{i \theta t}, & \Phi_{\dot{k}, m n}^{+}(t) & =\delta_{k n}(1-u(m-r)),
\end{aligned}
$$

where $u(x)$ is the unit step function

$$
u(x)=\left\{\begin{array}{lll}
0 & \text { for } & x<0 \\
1 & \text { for } & x \geq 0
\end{array} .\right.
$$

\section{$\phi_{0}$ and $\phi_{(r)}$ in terms of elementary free fields}

Then one gets $\tilde{\phi}_{(r)}$ which appears in the rank-r heighest weight bound state operator

$$
\begin{aligned}
\phi_{(r)}(\theta) & =\sum_{k=-\frac{r-1}{2}}^{\frac{r-1}{2}} \phi_{0}\left(\theta+\mathfrak{u}_{2 k}\right)+\tilde{\phi}_{(r)}(\theta), \\
\tilde{\phi}_{(r)}(\theta) & =\sum_{n=1}^{r-1} \sum_{k=-\frac{r-1}{2}}^{\frac{r-1}{2}-n}\left(\phi_{n \dot{n}}\left(\theta+\mathfrak{u}_{2 k}\right)+\phi_{\dot{n}, n+1}\left(\theta+\mathfrak{u}_{2 k}\right)\right), \\
\tilde{\phi}_{(r)}^{-}(\theta) & =\xi_{11}^{-}\left(\theta-\mathfrak{u}_{r+1}\right)-\xi_{11}^{-}\left(\theta+\mathfrak{u}_{r-1}\right)+\xi_{r r}^{-}\left(\theta-\mathfrak{u}_{r-1}\right)-\xi_{r r}^{-}\left(\theta-\mathfrak{u}_{r+1}\right) \\
\tilde{\phi}_{(r)}^{-}(\theta) & =\int_{0}^{\infty} \frac{d t}{i t} \tilde{\Phi}_{(r), m n}^{-}(t) a_{m n}(t) e^{i \theta t}, \\
\tilde{\Phi}_{(r), m n}^{-}(t) & =e^{\frac{\pi}{N} t}\left(e^{\frac{\pi r}{N} t}-e^{-\frac{\pi r}{N} t}\right) \delta_{1 m} \delta_{1 n}+e^{\frac{\pi r}{N} t}\left(e^{-\frac{\pi}{N} t}-e^{\frac{\pi}{N} t}\right) \delta_{r m} \delta_{r n} .
\end{aligned}
$$

Taking into account that

$$
\sum_{k=-\frac{r-1}{2}}^{\frac{r-1}{2}-n} \phi_{\mu}\left(\theta+\mathfrak{u}_{2 k}\right)=\int_{-\infty}^{\infty} \frac{d t}{i t} e^{\frac{\pi n}{N} t} \frac{\sinh \pi t \frac{r-n}{N}}{\sinh \frac{\pi t}{N}} \Phi_{\mu A}(t) a_{A}(t) e^{i \theta t}
$$

one gets

$$
\begin{aligned}
\tilde{\phi}_{(r)}^{+}(\theta) & =\int_{-\infty}^{0} \frac{d t}{i t} \tilde{\Phi}_{(r), m n}^{+}(t) a_{m n}(t) e^{i \theta t}, \\
\tilde{\Phi}_{(r), m n}^{+}(t) & =e^{\frac{\pi m}{N} t} \frac{\sinh \pi t \frac{r-m}{N}}{\sinh \frac{\pi t}{N}} u(r-1-m) u(r-1-n) u(m-1-n) \\
& +e^{\frac{\pi n}{N} t} \frac{\sinh \pi t \frac{r-n}{N}}{\sinh \frac{\pi t}{N}} u(r-1-m) u(r-1-n) u(n-m),
\end{aligned}
$$

and therefore

$$
\begin{aligned}
& \tilde{\phi}_{(r)}^{+}(\theta)=\int_{-\infty}^{0} \frac{d t}{i t} \sum_{m, n=0}^{r-1} f_{m n}^{(r)}(t) a_{m n}(t) e^{i \theta t}, \\
& f_{m n}^{(r)}(t)=\left\{\begin{array}{lll}
e^{\frac{\pi m}{N} t \frac{\sinh \pi t \frac{r-m}{N}}{\sinh \frac{\pi t}{N}}} & \text { for } \quad n<m<r \\
e^{\frac{\pi n}{N} t \frac{\sinh \pi t \frac{r-n}{N}}{\sinh \frac{\pi t}{N}}} & \text { for } \quad m \leq n<r
\end{array},\right.
\end{aligned}
$$




$$
\phi_{(r)}(\theta)=\int_{-\infty}^{\infty} \frac{d t}{i t} \frac{\sinh \pi t \frac{r}{N}}{\sinh \frac{\pi t}{N}} A_{0}(t) e^{i \theta t}+\tilde{\phi}_{(r)}(\theta) .
$$

Then the relation $V_{(N)}=1$ becomes $\phi_{(N)}=0$, and allows one to express $\phi_{0}^{ \pm}$in terms of $\xi_{k r}^{ \pm}$. Taking into account that $\xi_{N N}=0$, and that

$$
\sum_{k=-\frac{N-1}{2}}^{\frac{N-1}{2}} \phi_{0}\left(\theta+\mathfrak{u}_{2 k}\right)=\int_{-\infty}^{\infty} \frac{d t}{i t} \frac{\sinh \pi t}{\sinh \frac{\pi t}{N}} A_{0}(t) e^{i \theta t}=\int_{-\infty}^{\infty} \frac{d t}{i t} \frac{\sinh \pi t}{\sinh \frac{\pi t}{N}} \Phi_{0, m n}(t) a_{m n}(t) e^{i \theta t}
$$

one gets

$$
\begin{gathered}
\Phi_{0, m n}^{-}(t)=-2 e^{\frac{\pi}{N} t} \sinh \frac{\pi t}{N} \delta_{1 m} \delta_{1 n} \Rightarrow A_{0}^{-}(t)=-2 e^{\frac{\pi}{N} t} \sinh \frac{\pi t}{N} a_{11}(t), \\
\Phi_{0, m n}^{+}(t)=-\frac{\sinh \frac{\pi t}{N}}{\sinh \pi t} f_{m n}^{(N)}(t)=\left\{\begin{array}{lll}
-e^{\frac{\pi m}{N} t \frac{\sinh \pi t \frac{N-m}{N}}{\sinh \frac{\pi t}{N}}} & \text { for } n<m \\
-e^{\frac{\pi n}{N} t \frac{\sinh \pi t \frac{\pi}{N}}{\sinh \pi t}} & \text { for } n \geq m
\end{array} .\right.
\end{gathered}
$$

Thus,

$$
\begin{aligned}
{\left[A_{0}(t), A_{0}\left(t^{\prime}\right)\right] } & =\Phi_{0, m n}^{-}(t) \Phi_{0, m n}^{+}(-t) t \delta\left(t+t^{\prime}\right)=t f_{00}(t) \delta\left(t+t^{\prime}\right), \\
f_{00}(t) & =\Phi_{0, m n}^{-}(t) \Phi_{0, m n}^{+}(-t)=2 \frac{\sinh \frac{1}{N} \pi t \sinh \frac{N-1}{N} \pi t}{\sinh \pi t}, \quad t>0,
\end{aligned}
$$

as required. Moreover, by using the formulae for $A_{0}$, one gets

$$
\begin{gathered}
\phi_{(r)}^{-}(\theta)=\int_{0}^{\infty} \frac{d t}{i t} \Phi_{(r), m n}^{-}(t) a_{m n}(t) e^{i \theta t} \\
\Phi_{(r), m n}^{-}(t)=-2 e^{\frac{\pi r}{N} t} \sinh \frac{\pi}{N} t \delta_{r m} \delta_{r n} \Rightarrow A_{(r)}^{-}(t)=-2 e^{\frac{\pi r}{N} t} \sinh \frac{\pi t}{N} a_{r r}(t), \\
\phi_{(r)}^{+}(\theta)=\int_{-\infty}^{0} \frac{d t}{i t} \Phi_{(r), m n}^{+}(t) a_{m n}(t) e^{i \theta t}, \quad \Phi_{(r), m n}^{+}(t)=-\frac{\sinh \frac{\pi r}{N} t}{\sinh \pi t} f_{m n}^{(N)}(t)+\tilde{\Phi}_{(r), m n}^{+}(t) .
\end{gathered}
$$

Notice that $\phi_{(1)}=\phi_{0}$, and these formulae are consistent with the equality. In particular, one gets

$$
\begin{aligned}
{\left[A_{(r)}(t), A_{(r)}\left(t^{\prime}\right)\right] } & =\Phi_{(r), m n}^{-}(t) \Phi_{(r), m n}^{+}(-t) t \delta\left(t+t^{\prime}\right)=t f_{(r)(r)}(t) \delta\left(t+t^{\prime}\right), \quad t>0 \\
f_{(r)(r)}(t) & =\Phi_{(r), m n}^{-}(t) \Phi_{(r), m n}^{+}(-t)=2 \frac{\sinh \frac{r}{N} \pi t \sinh \frac{N-r}{N} \pi t}{\sinh \pi t}, \quad t>0,
\end{aligned}
$$

and in general

$$
\begin{aligned}
{\left[A_{(r)}(t), A_{(q)}\left(t^{\prime}\right)\right] } & =\Phi_{(r), m n}^{-}(t) \Phi_{(q), m n}^{+}(-t) t \delta\left(t+t^{\prime}\right)=t f_{(r)(q)}(t) \delta\left(t+t^{\prime}\right), \quad t>0, \\
f_{(r)(q)}(t) & =\Phi_{(r), r r}^{-}(t) \Phi_{(q), r r}^{+}(-t)=\left\{\begin{array}{ll}
2 \frac{\sinh \frac{q}{N} \pi t \sinh \frac{N-r}{N} \pi t}{\sinh \pi t} & \text { for } \quad r \geq q-q \\
2 \frac{\sinh \frac{r}{N} \pi t \sinh \frac{N-q}{N} \pi t}{\sinh \pi t} & \text { for } \quad r<q
\end{array}, t>0,\right.
\end{aligned}
$$


where it is used that

$$
2 \frac{\sinh \frac{q}{N} \pi t \sinh \frac{N-r}{N} \pi t}{\sinh \pi t}-2 \sinh \pi t \frac{q-r}{N}=2 \frac{\sinh \frac{r}{N} \pi t \sinh \frac{N-q}{N} \pi t}{\sinh \pi t} .
$$

Then, one gets

$$
g_{(r)(q)}(\theta)=g_{(q)(r)}(\theta), \quad g_{(r)(q)}(\theta)=\frac{\Gamma\left(\frac{i \theta}{2 \pi}-\frac{r+q}{2 N}+1\right) \Gamma\left(\frac{i \theta}{2 \pi}+\frac{r+q}{2 N}\right)}{\Gamma\left(\frac{i \theta}{2 \pi}-\frac{r-q}{2 N}+1\right) \Gamma\left(\frac{i \theta}{2 \pi}+\frac{r-q}{2 N}\right)}, \quad r \geq q .
$$

\section{ZF operators $Z_{2 \dot{1}}, Z_{1 \dot{2}}, Z_{2 \dot{2}}$}

It is easy to get $Z_{21}$ and $Z_{12}$

$$
\begin{aligned}
Z_{2 \dot{1}}(\theta) & =Z_{2 \dot{1}}^{(1)}(\theta)+Z_{2 \dot{1}}^{(2)}(\theta) \\
& =\rho(\theta) \int_{\theta^{--} / C_{1 \dot{r}}} c_{1 \dot{r}} g_{0 \mid \dot{1}}^{a} e^{i \phi_{0}+i \phi_{1 \dot{r}}}+\rho(\theta) c_{1 \dot{r}}(\theta) R_{0 \mid \dot{r}} e^{i \phi_{0}+i \phi_{1 \dot{r}}(\theta)} \\
Z_{1 \dot{2}}(\theta) & =Z_{1 \dot{2}}^{(1)}(\theta)+Z_{1 \dot{2}}^{(2)}(\theta) \\
& =\rho(\theta) \int_{\theta^{--} / C_{\mathrm{i} r}} c_{\dot{1} r} g_{0 \mid \dot{1} r}^{a} e^{i \phi_{0}+i \phi_{\mathrm{i} r}}+\rho(\theta) c_{\dot{1} r}(\theta) R_{0 \mid \dot{1} r} e^{i \phi_{0}(\theta)+i \dot{\phi}_{\mathrm{i} r}(\theta)} .
\end{aligned}
$$

Taking into account that $R_{0 \mid 1 \dot{r}}=\delta_{\dot{r} \mathrm{i}} R_{0 \mid 1 \mathrm{i}}$, and $R_{0 \mid \mathrm{i} r}=\delta_{r 1} R_{0 \mid \mathrm{i} 1}$, one obtains 4.10) and (4.11).

Before shifting the contours the operator $Z_{2 \dot{2}}$ is given by

$$
Z_{2 \dot{2}}(\theta)=\dot{\chi}_{\dot{1}}^{-} Z_{2 \dot{1}}(\theta)-Z_{2 \dot{1}}(\theta) \dot{\chi}_{\dot{1}}^{-}
$$

The second term does not require any shifting of the contours, and is given by

$$
\begin{aligned}
& -Z_{2 \mathrm{i}} \dot{\chi}_{\dot{1}}^{-}=-\rho(\theta) \int_{\theta^{--} / C_{1 \dot{r}} / C_{\mathrm{i}_{q}}} c_{1 \dot{r}} c_{\mathrm{1} q} g_{0 \mid 1 \dot{r}}^{a} g_{\mathrm{i} q \mid 0} g_{\dot{\mathrm{i} q \mid 1 \dot{r}}} e^{i \phi_{0}+i \phi_{1 \dot{r}}+i \dot{\phi}_{\mathrm{1} q}} \\
& -\rho(\theta) \int_{\theta^{--} / C_{\mathrm{i}_{q}}} c_{\mathrm{i} q} c_{1 \dot{r}}\left(\theta_{1 \dot{r}}\right) R_{0 \mid \dot{1} \dot{r}} g_{\mathrm{i} q \mid 0} g_{\mathrm{i} q \mid 1 \dot{r}}\left(\alpha_{\mathrm{i}_{q}}-\theta\right) e^{i \phi_{0}+i \phi_{1 \dot{r}}(\theta)+i \dot{\phi}_{\mathrm{i}_{q}}} .
\end{aligned}
$$

The first term is given by

$$
\begin{aligned}
& \dot{\chi}_{\dot{\mathrm{i}}}^{-} Z_{2 \mathrm{i}}=\dot{\chi}_{\mathrm{i}}^{-} Z_{2 \dot{1}}^{(1)}(\theta)+\dot{\chi}_{\mathrm{i}}^{-} Z_{2 \dot{1}}^{(2)}(\theta) \\
& =\rho(\theta) \int_{C_{\mathrm{1}_{q} / \theta / \theta^{--} / C_{1 \dot{r}}}} c_{1 \dot{r}} c_{\mathrm{1}_{q}} g_{0 \mid 1 \dot{r}}^{a} g_{0 \mid \dot{\mathrm{i} q}} g_{1 \dot{r} \mid \mathrm{i} q} e^{i \phi_{0}+i \phi_{1 \dot{r}}+i \dot{\phi}_{\mathrm{i} q}} \\
& +\rho(\theta) \int_{C_{\mathrm{i}_{q} / \theta}} c_{1 \dot{r}}(\theta) c_{\mathrm{1}_{q}} R_{0 \mid 1 \dot{r}} g_{0 \mid \dot{\mathrm{i}}_{q}}\left(\theta-\alpha_{\mathrm{i}_{q}}\right) g_{1 \dot{r} \mid \mathrm{i}_{q}}\left(\theta-\alpha_{\dot{1}_{q}}\right) e^{i \phi_{0}+i \phi_{1 \dot{r}}(\theta)+i \dot{\phi}_{\mathrm{i}_{q}}} .
\end{aligned}
$$

Shifting $C_{\mathrm{i}_{q}}$ down, one gets

$$
\begin{aligned}
& \dot{\chi}_{\dot{1}}^{-} Z_{2 \dot{1}}^{(1)}(\theta)=\rho(\theta) \int_{\theta^{--} / C_{1 \dot{r}} / C_{\dot{1} q}} c_{1 \dot{r}} c_{\dot{1} q} g_{0 \mid 1 \dot{r}}^{a} g_{0 \mid \dot{1} q} g_{\dot{1} q \mid 1 \dot{r}} e^{i \phi_{0}+i \phi_{1 \dot{r}}+i \dot{\phi}_{1 q}} \\
& +\rho(\theta) \int_{\theta^{--} / C_{1 \dot{r}}} c_{1 \dot{r}} c_{\dot{1} q}(\theta) g_{0 \mid 1 \dot{r}}^{a} R_{0 \mid \dot{1} q} g_{1 \dot{r} \mid \dot{q} q}\left(\alpha_{1 \dot{r}}-\theta\right) e^{i \phi_{0}+i \phi_{1 \dot{r}}+i \dot{\phi}_{\dot{1}_{q}}(\theta)} \\
& +\rho(\theta) \int_{\theta^{--} / C_{1 \dot{r}}} c_{1 \dot{r}} c_{\dot{1}_{q}}\left(\alpha_{1 \dot{r}}\right) g_{0 \mid 1 \dot{r}}^{a} g_{0 \mid \mathrm{i}_{q}}\left(\theta-\alpha_{1 \dot{r}}\right) R_{1 \dot{r} \mid \dot{1} q} e^{i \phi_{0}+i \phi_{1 \dot{r}}+i \dot{\phi}_{\mathrm{1}_{q}}\left(\alpha_{1 \dot{r}}\right)} .
\end{aligned}
$$


Here and in what follows the replacement $g_{1 \dot{r} \mid \dot{1}_{q}} \rightarrow g_{\dot{1} q \mid 1 \dot{r}}$ is done to stress that the contour $C_{\dot{1} q}$ is below $C_{1 \dot{r}}$. Finally, $\dot{\chi}_{\dot{1}}^{-} Z_{2 \dot{1}}^{(2)}$ is given by

$$
\begin{aligned}
& \dot{\chi}_{\dot{1}}^{-} Z_{2 \dot{1}}^{(2)}(\theta)=\rho(\theta) \int_{\theta^{--} / C_{\dot{1}_{q}}} c_{1 \dot{r}}(\theta) c_{\mathrm{i}_{q}} R_{0 \mid 1 \dot{r}} g_{0 \mid \dot{1} q}\left(\theta-\alpha_{\dot{1}_{q}}\right) g_{\mathrm{i} q \mid 1 \dot{r}}\left(\alpha_{\dot{1}_{q}}-\theta\right) e^{i \phi_{0}+i \phi_{1 \dot{r}}(\theta)+i \dot{\phi}_{1 q}} \\
& +\rho(\theta) c_{1 \dot{r}}(\theta) c_{\dot{1} q}(\theta) R_{0 \mid 1 \dot{r}} R_{0 \mid \dot{1} q} e^{i \phi_{0}+i \phi_{1 \dot{r}}(\theta)+i \dot{\phi}_{\dot{1}_{q}}(\theta)} \\
& +\rho(\theta) c_{1 \dot{r}}(\theta) c_{\dot{1} q}(\theta) R_{0 \mid 1 \dot{r}} R_{1 \dot{r} \mid \dot{1} q} e^{i \phi_{0}+i \phi_{1 \dot{r}}(\theta)+i \dot{\phi}_{\dot{1}_{q}}(\theta)},
\end{aligned}
$$

where in the last two terms one used that $R_{0 \mid 1 \dot{r}}=\delta_{\dot{r} \mathrm{i}} R_{0 \mid 1 \mathrm{i}}$ and $R_{0 \mid \mathrm{i} q}=\delta_{q 1} R_{0 \mid \mathrm{i} 1}$, and $g_{0 \mid 1 \dot{2}}=g_{11 \mid 1 \mathrm{i}}=1$. Summing up the terms, and taking into account that $R_{0 \mid 1 \dot{r}}=\delta_{\dot{r} \mathrm{i}} R_{0 \mid 1 \mathrm{i}}$ and $R_{0 \mid \mathrm{i} r}=\delta_{r 1} R_{0 \mid \mathrm{i} 1}$, one gets 4.12 .

\section{Traces of vertex operators}

\section{General formula}

To compute traces of products of vertex operators defined as

$$
V(\theta)=: \exp (i \phi(\theta)):
$$

where $\phi(\theta)$ is a linear combination of the $N^{2}-1$ elementary oscillators $a_{m n}(t)$

$$
\begin{gathered}
\phi(\theta)=\int_{-\infty}^{\infty} \frac{d t}{i t} \Phi_{A}(t) a_{A}(t) e^{i \theta t}=\int_{0}^{\infty} \frac{d t}{i t} \bar{\alpha}_{A}(t) a_{A}(t)-\int_{0}^{\infty} \frac{d t}{i t} a_{A}^{\dagger}(t) \beta_{A}(t), \\
\bar{\alpha}_{A}(t)=\Phi_{A}(t) e^{i \theta t}, \quad \beta_{A}(t)=\Phi_{A}(-t) e^{-i \theta t}, \quad a_{A}^{\dagger}(t) \equiv a_{A}(-t),
\end{gathered}
$$

it is sufficient to know how to compute

$$
\operatorname{Tr}_{F}(\exp (2 \pi i K) V), \quad K=i H=i \int_{0}^{\infty} d t \sum_{A} a_{A}^{\dagger}(t) a_{A}(t)
$$

where $F$ is the Fock space where $a_{A}(t)$ act, and

$$
V=e^{i \phi}, \quad \phi=\int_{0}^{\infty} \frac{d t}{i t} \bar{\alpha}_{A}(t) a_{A}(t)-\int_{0}^{\infty} \frac{d t}{i t} a_{A}^{\dagger}(t) \beta_{A}(t)
$$

The formula takes the following form

$$
\frac{\operatorname{Tr}_{F}(\exp (2 \pi i K) V)}{\operatorname{Tr}_{F}(\exp (2 \pi i K))}=\exp \left(\int_{0}^{\infty} \frac{d t}{t} \frac{\bar{\alpha}_{A}(t) \beta_{A}(t)}{1-e^{2 \pi t}}\right)
$$

and its derivation can be found in e.g. [27]. This formula agrees with the prescription in [19]. To show this let's consider

$$
\operatorname{Tr}_{F}\left(\exp (2 \pi i K) V_{2} V_{1}\right)
$$


where

$$
V_{k}=: \exp \left(i \phi_{k}\right):, \quad \phi_{k}=\int_{0}^{\infty} \frac{d t}{i t} \bar{\alpha}_{A}^{(k)}(t) a_{A}(t)-\int_{0}^{\infty} \frac{d t}{i t} a_{A}^{\dagger}(t) \beta_{A}^{(k)}(t) .
$$

Then one gets

$$
V_{2} V_{1}=g_{12}: V_{2} V_{1}:, \quad g_{12}=\exp \left(-\int_{0}^{\infty} \frac{d t}{t} \bar{\alpha}_{A}^{(2)}(t) \beta_{A}^{(1)}(t)\right),
$$

and

$$
\frac{\operatorname{Tr}_{F}\left(\exp (2 \pi i K) V_{2} V_{1}\right)}{\operatorname{Tr}_{F}(\exp (2 \pi i K))}=\exp \left(\int_{0}^{\infty} \frac{d t}{t}\left(-\bar{\alpha}_{A}^{(2)}(t) \beta_{A}^{(1)}(t)+\frac{\bar{\alpha}_{A}(t) \beta_{A}(t)}{1-e^{2 \pi t}}\right)\right)
$$

where

$$
\bar{\alpha}_{A}=\bar{\alpha}_{A}^{(1)}+\bar{\alpha}_{A}^{(2)}, \quad \beta_{A}(t)=\beta_{A}^{(1)}+\beta_{A}^{(2)}
$$

Thus,

$$
\frac{\operatorname{Tr}_{F}\left(\exp (2 \pi i K) V_{2} V_{1}\right)}{\operatorname{Tr}_{F}(\exp (2 \pi i K))}=C_{1} C_{2} G_{12}
$$

where

$$
C_{k}=\frac{\operatorname{Tr}_{F}\left(\exp (2 \pi i K) V_{k}\right)}{\operatorname{Tr}_{F}(\exp (2 \pi i K))}
$$

and

$$
G_{12}=\exp \left(-\int_{0}^{\infty} \frac{d t}{t}\left(\frac{\bar{\alpha}_{A}^{(1)}(t) \beta_{A}^{(2)}(t)}{e^{2 \pi t}-1}+\frac{\bar{\alpha}_{A}^{(2)}(t) \beta_{A}^{(1)}(t)}{1-e^{-2 \pi t}}\right)\right)
$$

Introducing

$$
\left\langle\left\langle a_{A}(t) a_{B}\left(t^{\prime}\right)\right\rangle\right\rangle=\frac{t \delta_{A B}}{1-e^{-2 \pi t}} \delta\left(t+t^{\prime}\right),
$$

one finds

$$
\left\langle\left\langle\phi_{2} \phi_{1}\right\rangle\right\rangle=-\log G_{12} .
$$

The generalisation to the product of $n$ vertex operators

$$
U_{j}(\theta)=: e^{i \phi_{j}(\theta)}:=e^{i \phi_{j}^{+}(\theta)} e^{i \phi_{j}^{-}(\theta)},
$$

is straightforward, and one gets

$$
\left\langle\left\langle U_{n}\left(\theta_{n}\right) \cdots U_{1}\left(\theta_{1}\right)\right\rangle\right\rangle=\prod_{j=1}^{n} C_{U_{j}} \prod_{k<j} G_{U_{k} U_{j}}\left(\theta_{k}-\theta_{j}\right),
$$

where

$$
\begin{gathered}
C_{U_{j}}=\left\langle\left\langle U_{j}\left(\theta_{j}\right)\right\rangle\right\rangle=\exp \left(-\left\langle\left\langle\phi_{j}^{-}(0) \phi_{j}^{+}(0)\right\rangle\right\rangle\right), \\
G_{U_{k} U_{j}}\left(\theta_{k}-\theta_{j}\right)=\exp \left(-\left\langle\left\langle\phi_{j}\left(\theta_{j}\right) \phi_{k}\left(\theta_{k}\right)\right\rangle\right\rangle\right) .
\end{gathered}
$$




\section{Traces of single $V$ 's}

The traces of the vertex operators of the fields $\phi_{0}, \phi_{(r)}, \phi_{k r}, \phi_{k r}$ are given by

$$
C_{\mu}=\frac{\operatorname{Tr}_{F}\left(e^{2 \pi i K} V_{\mu}\right)}{\operatorname{Tr}_{F}\left(e^{2 \pi i K}\right)}=\exp \left(\int_{0}^{\infty} \frac{d t}{t} \frac{\Phi_{\mu, A}^{-}(t) \Phi_{\mu, A}^{+}(-t)}{1-e^{2 \pi t}}\right)=\exp \left(\int_{0}^{\infty} \frac{d t}{t} \frac{f_{\mu \mid \mu}(t)}{1-e^{2 \pi t}}\right)
$$

where

$$
f_{\nu \mid \mu}(t) \equiv \Phi_{\mu, A}^{-}(t) \Phi_{\nu, A}^{+}(-t), \quad t>0
$$

The functions $f_{\mu \mid \mu}(t)$ are given by

$$
\begin{gathered}
f_{0 \mid 0}(t)=\Phi_{0, m n}^{-}(t) \Phi_{0, m n}^{+}(-t)=2 \frac{\sinh \frac{1}{N} \pi t \sinh \frac{N-1}{N} \pi t}{\sinh \pi t} \\
f_{(r) \mid(r)}(t)=\Phi_{(r), m n}^{-}(t) \Phi_{(r), m n}^{+}(-t)=2 \frac{\sinh \frac{r}{N} \pi t \sinh \frac{N-r}{N} \pi t}{\sinh \pi t} \\
f_{k \dot{r} \mid k \dot{r}}(t)=\Phi_{k \dot{r}, m n}^{-}(t) \Phi_{k \dot{r}, m n}^{+}(-t)=1-e^{-2 \pi t / N} \\
f_{\dot{k} r \mid k r}(t)=\Phi_{\dot{k}, m n}^{-}(t) \Phi_{\dot{k}, m n}^{+}(-t)=1-e^{-2 \pi t / N}
\end{gathered}
$$

Notice that all these functions asymptote to 1 at large $t$, and therefore the integrals are well-defined, and can be computed explicitly by using formulae from appendix B of [27].

\section{Traces of $V_{\mu} V_{\nu}$ and functions $G_{\mu \nu}$}

To compute the traces one uses (D.12), and (D.14) which takes the form

$$
G_{\nu \mid \mu}\left(\beta_{2}-\beta_{1}\right)=\exp \left(-\left\langle\left\langle\phi_{\mu}\left(\beta_{1}\right) \phi_{\nu}\left(\beta_{2}\right)\right\rangle\right\rangle\right)
$$

and therefore

$$
G_{\nu \mid \mu}(\beta)=\exp \left(-\int_{0}^{\infty} \frac{d t}{t}\left(\frac{f_{\nu \mid \mu}(t) e^{-i \beta t}}{1-e^{-2 \pi t}}+\frac{f_{\mu \mid \nu}(t) e^{i \beta t}}{e^{2 \pi t}-1}\right)\right) .
$$

These satisfy the relations

$$
G_{\mu \mid \nu}(\beta-2 \pi i)=G_{\nu \mid \mu}(-\beta), \quad S_{\mu \mid \nu}(\beta)=\frac{G_{\nu \mid \mu}(-\beta)}{G_{\mu \mid \nu}(\beta)}=\frac{G_{\mu \mid \nu}(\beta-2 \pi i)}{G_{\mu \mid \nu}(\beta)},
$$

which are necessary to satisfy the form factors axioms.

If $f_{\nu \mid \mu}(t)=f_{\mu \mid \nu}(t)$, in particular, for $\nu=\mu$, one gets the familiar representation

$$
G_{\nu \mid \mu}(\beta)=G_{\mu \mid \nu}(\beta)==\exp \left(-\int_{0}^{\infty} \frac{d t}{t} \frac{f_{\nu \mid \mu}(t) \cos (\beta+i \pi) t}{\sinh \pi t}\right), \quad f_{\nu \mid \mu}(t)=f_{\mu \mid \nu}(t) .
$$


Thus, taking into account that $f_{(r)(q)}(t)=f_{(q)(r)}(t)$, one gets the following representations for $G_{(r) \mid(q)}$ (note that $G_{0(q)} \equiv G_{(1) \mid(q)}$ )

$$
G_{(r) \mid(q)}(\beta)=\exp \left(-2 \int_{0}^{\infty} \frac{d t}{t} \frac{\sinh \frac{q}{N} \pi t \sinh \frac{N-r}{N} \pi t \cos (\beta+i \pi) t}{\sinh ^{2} \pi t}\right), \quad r \geq q,
$$

which is well-defined for

$$
-2 \pi-\frac{|r-q|}{N} \pi<\operatorname{Im}(\beta)<\frac{|r-q|}{N} \pi .
$$

The functions $G_{k \dot{r} \mid k \dot{r}}$ and $G_{\dot{k} \mid \dot{k} r}$ are equal to each other

$$
G_{k \dot{r} \mid k \dot{r}}(\beta)=G_{\dot{k}_{r \mid k r}}(\beta)=\exp \left(-\int_{0}^{\infty} \frac{d t}{t} \frac{\left(1-e^{-2 \pi t / N}\right) \cos (\beta+i \pi) t}{\sinh \pi t}\right),
$$

and computing them by using formulae from appendix B of [27], one gets

$$
G_{k \dot{r} \mid k \dot{r}}(\beta)=e^{2 \gamma / N}(2 \pi)^{2 / N} \frac{i}{\pi} \Gamma\left(\frac{1}{N}+\frac{i \beta}{2 \pi}\right) \Gamma\left(1+\frac{1}{N}-\frac{i \beta}{2 \pi}\right) \sinh \frac{\beta}{2} .
$$

The function has poles at

$$
\beta=\frac{2 \pi}{N} i+2 \pi i m \text { and } \beta=-\frac{2 \pi(N+1)}{N} i-2 \pi i m, \quad m=0,1,2, \ldots,
$$

and the integration contour in $\beta$ should run below the poles $\beta=2 \pi i / N+2 \pi i m$ but above $\beta=-\frac{2 \pi(N+1)}{N} i-2 \pi i m$ because the only pole of the function $g_{k \dot{r} \mid k \dot{r}}(\beta)$ is at $\beta=\frac{2 \pi}{N} i$.

One also finds

$$
\begin{aligned}
f_{0 \mid 1 \mathrm{i}}(t) & =f_{0 \mid \mathrm{i} 1}(t)=e^{-2 \pi t / N}-1, \quad f_{1 \mathrm{i} \mid 0}(t)=f_{\mathrm{i} 1 \mid 0}(t)=0, \\
G_{0 \mid 1 \mathrm{i}}(\beta) & =G_{0 \mid \mathrm{i} 1}(\beta)=\exp \left(-\int_{0}^{\infty} \frac{d t}{t} \frac{\left(e^{-2 \pi t / N}-1\right) e^{-i \beta t}}{1-e^{-2 \pi t}}\right) \\
& =e^{-\gamma / N}(2 \pi)^{-1 / N} \frac{\Gamma\left(\frac{i \beta}{2 \pi}\right)}{\Gamma\left(\frac{1}{N}+\frac{i \beta}{2 \pi}\right)},
\end{aligned}
$$

$G_{0 \mid 1 \mathrm{i}}(\beta)$ has poles at $\beta=2 \pi i m$, and in the product of Green's functions containing $G_{0 \mid 1 \mathrm{i}}\left(\theta-\alpha_{1 \mathrm{i}}\right)$ the integration contour $C_{\alpha_{1 \mathrm{i}}}$ runs above the poles at $\alpha_{1 \mathrm{i}}=\theta-2 \pi i \mathrm{~m}$.

$$
\begin{aligned}
G_{1 \mathrm{i} \mid 0}(\beta) & =G_{\mathrm{i} 1 \mid 0}(\beta)=\exp \left(-\int_{0}^{\infty} \frac{d t}{t} \frac{\left(e^{-2 \pi t / N}-1\right) e^{i \beta t}}{e^{2 \pi t}-1}\right) \\
& =e^{-\gamma / N}(2 \pi)^{-1 / N} \frac{\Gamma\left(1-\frac{i \beta}{2 \pi}\right)}{\Gamma\left(1+\frac{1}{N}-\frac{i \beta}{2 \pi}\right)} .
\end{aligned}
$$


$G_{1 \mathrm{i} \mid 0}(\beta)$ has poles at $\beta=-2 \pi i m, m=1,2, \ldots$, and in the product of Green's functions containing $G_{1 \mathrm{i} \mid 0}\left(\alpha_{1 \mathrm{i}}-\theta\right)$ the integration contour $C_{\alpha_{1 \mathrm{i}}}$ runs above all these poles at $\alpha_{1 i}=\theta-2 \pi i m$. Then

$$
G_{0 \mid 1 \mathrm{i}}^{a}(\beta)=G_{0 \mid 1 \mathrm{i}}(\beta)-G_{1 \mathrm{i} \mid 0}(-\beta)=\frac{e^{-\gamma / N}(2 \pi)^{-1 / N} \Gamma\left(\frac{i \beta}{2 \pi}\right)}{N \Gamma\left(1+\frac{1}{N}+\frac{i \beta}{2 \pi}\right)},
$$

and the integration contour $C_{\alpha_{11}}$ runs above its poles at $\alpha_{11}=\theta-2 \pi i m$.

$$
\begin{aligned}
f_{0 \mid 1 \dot{r}}(t) & =f_{0 \mid \dot{\mid} r}(t)=0, \quad f_{1 \dot{r} \mid 0}(t)=f_{\dot{1} r \mid 0}(t)=1-e^{2 \pi t / N}, \quad r, \dot{r} \geq 2, \\
G_{1 \dot{r} \mid 0}(\beta) & =G_{\dot{1} r \mid 0}(\beta)=\exp \left(-\int_{0}^{\infty} \frac{d t}{t} \frac{\left(1-e^{2 \pi t / N}\right) e^{-i \beta t}}{1-e^{-2 \pi t}}\right) \\
& =e^{-\gamma / N}(2 \pi)^{-1 / N} \frac{\Gamma\left(-\frac{1}{N}+\frac{i \beta}{2 \pi}\right)}{\Gamma\left(\frac{i \beta}{2 \pi}\right)},
\end{aligned}
$$

$G_{1 \dot{r} \mid 0}(\beta)$ has poles at $\beta=-2 \pi i / N+2 \pi i m$, and in the product of Green's functions containing $G_{1 \dot{r} \mid 0}\left(\alpha_{1 \dot{r}}-\theta\right)$ the integration contour $C_{\alpha_{1 \dot{r}}}$ runs below all these poles at $\alpha_{1 \dot{r}}=\theta-2 \pi i / N+2 \pi i m$.

$$
\begin{aligned}
G_{0 \mid 1 \dot{r}}(\beta) & =G_{0 \mid \dot{1} r}(\beta)=\exp \left(-\int_{0}^{\infty} \frac{d t}{t} \frac{\left(1-e^{2 \pi t / N}\right) e^{i \beta t}}{e^{2 \pi t}-1}\right) \\
& =e^{-\gamma / N}(2 \pi)^{-1 / N} \frac{\Gamma\left(1-\frac{1}{N}-\frac{i \beta}{2 \pi}\right)}{\Gamma\left(1-\frac{i \beta}{2 \pi}\right)},
\end{aligned}
$$

$G_{0 \mid 1 \dot{r}}(\beta)$ has poles at $\beta=-2 \pi i(N-1) / N-2 \pi i m$, and in the product of Green's functions containing $G_{0 \mid 1 \dot{r}}\left(\theta-\alpha_{1 \dot{r}}\right)$ the integration contour $C_{\alpha_{1 \dot{r}}}$ runs below all these poles at $\alpha_{1 \dot{r}}=\theta+2 \pi i(N-1) / N+2 \pi i m$. Then

$$
G_{0 \mid 1 \dot{r}}^{a}(\beta)=G_{0 \mid 1 \dot{r}}(\beta)-G_{1 \dot{r} \mid 0}(-\beta)=\frac{e^{-\gamma / N}(2 \pi)^{-1 / N} \Gamma\left(-\frac{1}{N}-\frac{i \beta}{2 \pi}\right)}{N \Gamma\left(1-\frac{i \beta}{2 \pi}\right)},
$$

and the integration contour $C_{\alpha_{1 \dot{r}}}$ in $G_{0 \mid 1 \dot{r}}^{a}\left(\theta-\alpha_{1 \dot{r}}\right)$ runs below its poles at $\alpha_{1 \dot{r}}=\theta-$ $2 \pi i / N+2 \pi i m$.

$$
\begin{aligned}
f_{k \dot{r} \mid k \dot{q}}(t) & =f_{\dot{k} r \mid \dot{k} q}(t)=0, \quad f_{k \dot{q} \mid k \dot{r}}(t)=f_{\dot{k} q \mid k r}(t)=e^{2 \pi t / N}-e^{-2 \pi t / N}, \quad r<q, \dot{r}<\dot{q}, \\
G_{k \dot{r} \mid k \dot{q}}(\beta) & =G_{\dot{k}_{r \mid k} q}(\beta)=\exp \left(-\int_{0}^{\infty} \frac{d t}{t} \frac{\left(e^{2 \pi t / N}-e^{-2 \pi t / N}\right) e^{i \beta t}}{e^{2 \pi t}-1}\right) \\
& =e^{-\gamma / N}(2 \pi)^{-1 / N} \frac{\Gamma\left(1-\frac{1}{N}-\frac{i \beta}{2 \pi}\right)}{\Gamma\left(1-\frac{i \beta}{2 \pi}\right)}
\end{aligned}
$$


$G_{k \dot{r} \mid k \dot{q}}(\beta), \dot{r}<\dot{q}$ has poles at $\beta=-2 \pi i(N-1) / N-2 \pi i m$, and in the product of Green's functions containing $G_{k \dot{r} \mid k \dot{q}}\left(\alpha_{k \dot{r}}-\alpha_{k \dot{q}}\right)$ the integration contour $C_{\alpha_{k \dot{q}}}$ runs below all these poles at $\alpha_{k \dot{q}}=\alpha_{k \dot{r}}+2 \pi i(N-1) / N+2 \pi i m$.

$$
\begin{aligned}
G_{k \dot{q} \mid k \dot{r}}(\beta) & =G_{\dot{k} q \mid k r}(\beta)=\exp \left(-\int_{0}^{\infty} \frac{d t}{t} \frac{\left(e^{2 \pi t / N}-e^{-2 \pi t / N}\right) e^{-i \beta t}}{1-e^{-2 \pi t}}\right) \\
& =e^{2 \gamma / N}(2 \pi)^{2 / N} \frac{\Gamma\left(\frac{1}{N}+\frac{i \beta}{2 \pi}\right)}{\Gamma\left(-\frac{1}{N}+\frac{i \beta}{2 \pi}\right)},
\end{aligned}
$$

$G_{k \dot{q} \mid k \dot{r}}(\beta), \dot{r}<\dot{q}$ has poles at $\beta=2 \pi i / N+2 \pi i m$, and in the product of Green's functions containing $G_{k \dot{q} \mid k \dot{r}}\left(\alpha_{k \dot{q}}-\alpha_{k \dot{r}}\right)$ the integration contour $C_{\alpha_{k \dot{q}}}$ runs below all these poles at $\alpha_{k \dot{q}}=\alpha_{k \dot{r}}+2 \pi i / N+2 \pi i m$.

$$
\begin{aligned}
& \left\{\begin{array}{l}
G_{k \dot{r} \mid k+1, \dot{q}}(\beta)=G_{\dot{k r} \mid \dot{k}+\dot{\mathrm{i}}, q}(\beta)=G_{0 \mid 1 \mathrm{i}}(\beta), \\
G_{k+1, \dot{q} \mid k \dot{r}}(\beta)=G_{\dot{k}+\dot{\mathrm{i}}, q \mid \dot{k} r}(\beta)=G_{1 \mathrm{i} \mid 0}(\beta), \quad \dot{r} \geq \dot{q}, r \geq q,
\end{array}\right. \\
& \left\{\begin{array}{l}
G_{k \dot{r} \mid k+1, \dot{q}}(\beta)=G_{\dot{k} \mid \dot{k}+\dot{1}, q}(\beta)=G_{0 \mid 1 \dot{2}}(\beta), \\
G_{k+1, \dot{q} \mid k \dot{r}}(\beta)=G_{\dot{k}+\dot{1}, q \mid k r}(\beta)=G_{1 \dot{2} \mid 0}(\beta), \quad \dot{r}<\dot{q}, r<q,
\end{array}\right. \\
& f_{k \dot{r} \mid \dot{r}, k+1}(t)=f_{\dot{r} k \mid k, \dot{r}+\dot{1}}(t)=e^{-2 \pi t / N}-1, \quad f_{\dot{r}, k+1 \mid k \dot{r}}(t)=f_{k, \dot{r}+\dot{1} \mid \dot{r} k}(t)=e^{2 \pi t / N}-1, \\
& G_{k \dot{r} \mid \dot{r}, k+1}(\beta)=G_{\dot{r} k \mid k, \dot{r}+\mathrm{i}}(\beta)=\exp \left(-\int_{0}^{\infty} \frac{d t}{t}\left(\frac{\left(e^{-2 \pi t / N}-1\right) e^{-i \beta t}}{1-e^{-2 \pi t}}+\frac{\left(e^{2 \pi t / N}-1\right) e^{i \beta t}}{e^{2 \pi t}-1}\right)\right) \\
& =\frac{G_{0 \mid 1 \mathrm{i}}(\beta)}{G_{0 \mid 1 \dot{2}}(\beta)}=\frac{\sinh \left(\frac{\beta}{2}-\frac{\pi i}{N}\right)}{\sinh \left(\frac{\beta}{2}\right)}, \\
& G_{\dot{r}, k+1 \mid k \dot{r}}(\beta)=G_{k, \dot{r}+\dot{i} \mid \dot{r} k}(\beta)=\exp \left(-\int_{0}^{\infty} \frac{d t}{t}\left(\frac{\left(e^{2 \pi t / N}-1\right) e^{-i \beta t}}{1-e^{-2 \pi t}}+\frac{\left(e^{-2 \pi t / N}-1\right) e^{i \beta t}}{e^{2 \pi t}-1}\right)\right) \\
& =\frac{\sinh \left(\frac{\beta}{2}+\frac{\pi i}{N}\right)}{\sinh \left(\frac{\beta}{2}\right)} .
\end{aligned}
$$

These functions have poles at $\beta=2 \pi i m, m \in \mathbb{Z}$. The contour should run below the poles with nonpositive imaginary part but above the poles with negative imaginary part.

$$
\begin{aligned}
f_{r \dot{q} \mid(r)}(t) & =f_{\dot{r} q \mid(r)}(t)=e^{(r-1) \pi t / N}-e^{(r+1) \pi t / N}, \quad f_{(r) \mid r \dot{q}}(t)=f_{(r) \mid \dot{r}}(t)=0, \quad \dot{q}>\dot{r}, \\
G_{r \dot{q} \mid(r)}(\beta) & =G_{\dot{r} q \mid(r)}(\beta)=\exp \left(-\int_{0}^{\infty} \frac{d t}{t} \frac{\left(e^{(r-1) \pi t / N}-e^{(r+1) \pi t / N}\right) e^{-i \beta t}}{1-e^{-2 \pi t}}\right) \\
& =e^{-\gamma / N}(2 \pi)^{-1 / N} \frac{\Gamma\left(-\frac{r+1}{2 N}+\frac{i \beta}{2 \pi}\right)}{\Gamma\left(-\frac{r-1}{2 N}+\frac{i \beta}{2 \pi}\right)},
\end{aligned}
$$


$G_{r \dot{q} \mid(r)}(\beta), \dot{q}>\dot{r}$ has poles at $\beta=-\pi i(r+1) / N+2 \pi i m$, and in the product of Green's functions containing $G_{r \dot{q} \mid(r)}\left(\alpha_{r \dot{q}}-\theta\right)$ the integration contour $C_{\alpha_{r \dot{q}}}$ runs below all these poles at $\alpha_{r \dot{q}}=\theta-\pi i(r+1) / N+2 \pi i m$.

$$
\begin{aligned}
G_{(r) \mid r \dot{q}}(\beta) & =G_{(r) \mid \dot{r} q}(\beta)=\exp \left(-\int_{0}^{\infty} \frac{d t}{t} \frac{\left(e^{(r-1) \pi t / N}-e^{(r+1) \pi t / N}\right) e^{i \beta t}}{e^{2 \pi t}-1}\right) \\
& =e^{-\gamma / N}(2 \pi)^{-1 / N} \frac{\Gamma\left(1-\frac{r+1}{2 N}-\frac{i \beta}{2 \pi}\right)}{\Gamma\left(1-\frac{r-1}{2 N}-\frac{i \beta}{2 \pi}\right)},
\end{aligned}
$$

$G_{(r) \mid r \dot{q}}(\beta), \dot{q}>\dot{r}$ has poles at $\beta=\pi i(r+1) / N-2 \pi i m, m=1,2, \ldots$, and in the product of Green's functions containing $G_{(r) \mid r \dot{q}}\left(\theta-\alpha_{r \dot{q}}\right)$ the integration contour $C_{\alpha_{r \dot{q}}}$ runs below all these poles at $\alpha_{r \dot{q}}=\theta-\pi i(r+1) / N+2 \pi i m$.

$$
\begin{aligned}
f_{r \dot{q} \mid(r)}(t) & =f_{\dot{r} q \mid(r)}(t)=0, \quad f_{(r) \mid r \dot{q}}(t)=f_{(r) \mid \dot{r} q}(t)=e^{-(r+1) \pi t / N}-e^{-(r-1) \pi t / N}, \quad \dot{q} \leq \dot{r}, \\
G_{r \dot{q} \mid(r)}(\beta) & =G_{\dot{r} q \mid(r)}(\beta)=\exp \left(-\int_{0}^{\infty} \frac{d t}{t} \frac{\left(e^{-(r+1) \pi t / N}-e^{-(r-1) \pi t / N}\right) e^{i \beta t}}{e^{2 \pi t}-1}\right) \\
& =e^{-\gamma / N}(2 \pi)^{-1 / N} \frac{\Gamma\left(1+\frac{r-1}{2 N}-\frac{i \beta}{2 \pi}\right)}{\Gamma\left(1+\frac{r+1}{2 N}-\frac{i \beta}{2 \pi}\right)},
\end{aligned}
$$

$G_{r \dot{q} \mid(r)}(\beta), \dot{q} \leq \dot{r}$ has poles at $\beta=-\pi i(r-1) / N-2 \pi i m, m=1,2, \ldots$, and in the product of Green's functions containing $G_{r \dot{q} \mid(r)}\left(\alpha_{r \dot{q}}-\theta\right)$ the integration contour $C_{\alpha_{r \dot{q}}}$ runs above all these poles at $\alpha_{r \dot{q}}=\theta-\pi i(r-1) / N-2 \pi i m$.

$$
\begin{aligned}
G_{(r) \mid r \dot{q}}(\beta) & =G_{(r) \mid \dot{r} q}(\beta)=\exp \left(-\int_{0}^{\infty} \frac{d t}{t} \frac{\left(e^{-(r+1) \pi t / N}-e^{-(r-1) \pi t / N}\right) e^{-i \beta t}}{1-e^{-2 \pi t}}\right) \\
& =e^{-\gamma / N}(2 \pi)^{-1 / N} \frac{\Gamma\left(\frac{r-1}{2 N}+\frac{i \beta}{2 \pi}\right)}{\Gamma\left(\frac{r+1}{2 N}+\frac{i \beta}{2 \pi}\right)},
\end{aligned}
$$

$G_{(r) \mid r \dot{q}}(\beta), \dot{q} \leq \dot{r}$ has poles at $\beta=\pi i(r-1) / N+2 \pi i m$, and in the product of Green's functions containing $G_{(r) \mid r \dot{q}}\left(\theta-\alpha_{r \dot{q}}\right)$ the integration contour $C_{\alpha_{r \dot{q}}}$ runs above all these poles at $\alpha_{r \dot{q}}=\theta-\pi i(r-1) / N-2 \pi i m$.

\section{References}

[1] M. Karowski and P. Weisz, "Exact Form-Factors in (1+1)-Dimensional Field Theoretic Models with Soliton Behavior," Nucl. Phys. B 139 (1978) 455.

[2] F. A. Smirnov, "The Quantum Gelfand-levitan-marchenko Equations And Formfactors In The Sine-gordon Model," J. Phys. A 17 (1984) L873.

[3] F. A. Smirnov, "Form-factors in completely integrable models of quantum field theory," Adv. Ser. Math. Phys. 14 (1992) 1. 
[4] F. A. Smirnov, "A New set of exact form-factors," Int. J. Mod. Phys. A 9 (1994) 5121 hep-th/9312039.

[5] H. M. Babujian, A. Fring, M. Karowski and A. Zapletal, "Exact form-factors in integrable quantum field theories: The Sine-Gordon model," Nucl. Phys. B 538 (1999) 535 [hep-th/9805185].

[6] H. Babujian and M. Karowski, "Exact form-factors in integrable quantum field theories: The sine-Gordon model. 2.," Nucl. Phys. B 620 (2002) 407 [hep-th/0105178].

[7] A. Nakayashiki and Y. Takeyama, "On form-factors of SU(2) invariant Thirring model," math-ph/0105040.

[8] Y. Takeyama, "Form-factors of SU(N) invariant Thirring model," Publ. Res. Inst. Math. Sci. Kyoto 39 (2003) 59 math-ph/0112025.

[9] H. M. Babujian, A. Foerster and M. Karowski, "The Nested SU(N) off-shell Bethe ansatz and exact form-factors," J. Phys. A 41 (2008) 275202 hep-th/0611012.

[10] H. Babujian, A. Foerster and M. Karowski, "Exact form factors of the SU(N) GrossNeveu model and 1/N expansion," Nucl. Phys. B 825 (2010) 396 arXiv:0907.0662 [hep-th]].

[11] H. M. Babujian, A. Foerster and M. Karowski, "Bethe Ansatz and exact form factors of the $O(N)$ Gross Neveu-model," JHEP 1602 (2016) 042 doi:10.1007/JHEP02(2016)042 arXiv:1510.08784 [hep-th]].

[12] S. L. Lukyanov, "Free field representation for massive integrable models," Commun. Math. Phys. 167 (1995) 183 hep-th/9307196].

[13] S. L. Lukyanov and S. L. Shatashvili, "Free field representation for the classical limit of quantum Affine algebra," Phys. Lett. B 298 (1993) 111 [hep-th/9209130].

[14] A. B. Zamolodchikov and A. B. Zamolodchikov, "Factorized S-matrices in two dimensions as the exact solutions of certain relativistic quantum field models," Annals Phys. 120 (1979) 253.

[15] L. D. Faddeev, Sov.Sci.Rev.Math.Phys. 1C(1980) 107.

[16] S. L. Lukyanov, "Form-factors of exponential fields in the Sine-Gordon model," Mod. Phys. Lett. A 12 (1997) 2543 [hep-th/9703190].

[17] S. L. Lukyanov, "Form-factors of exponential fields in the affine $A_{N-1}^{(1)}$ Toda model," Phys. Lett. B 408 (1997) 192 hep-th/9704213].

[18] V. Brazhnikov and S. L. Lukyanov, "Angular quantization and form-factors in massive integrable models," Nucl. Phys. B 512 (1998) 616 [hep-th/9707091]. 
[19] V. A. Fateev and M. Lashkevich, "Form-factors of exponential fields for two parametric family of integrable models," Nucl. Phys. B 696 (2004) 301 hep-th/0402082].

[20] O. Alekseev and M. Lashkevich, "Form factors of descendant operators: $A_{L-1}^{(1)}$ affine Toda theory," hep-th/09125225].

[21] M. Lashkevich, "Boundary form factors in the Smirnov-Fateev model with a diagonal boundary $S$ matrix," [hep-th/08010935].

[22] B. Feigin and M. Lashkevich, "Form factors of descendant operators: Free field construction and reflection relations," J. Phys. A 42 (2009) 304014 [mathph/08124776].

[23] O. Alekseev, "Form factors of descendant operators in the Bullough-Dodd model," hep-th/12102813].

[24] Z. Horvath and G. Takacs, "Free field representation for the $\mathrm{O}(3)$ nonlinear sigma model and bootstrap fusion," Phys. Rev. D 53 (1996) 3272-3284 hep-th/9501006.

[25] Z. Horvath and G. Takacs, "Form-factors of the sausage model obtained with bootstrap fusion from sine-Gordon theory," Phys. Rev. D 51 (1995) 2922-2932 |hepth/9601040.

[26] T. Kojima, "The SU(n) invariant massive Thirring model with boundary reflection," Int. J. Mod. Phys. A 16 (2001) 2665 [nlin/0010020 [nlin-si]].

[27] S. Britton and S. Frolov, "Free field representation and form factors of the chiral Gross-Neveu model," JHEP 1311 (2013) 076 doi:10.1007/JHEP11(2013)076 arXiv:1305.6252 [hep-th]].

[28] D. J. Gross and A. Neveu, "Dynamical Symmetry Breaking in Asymptotically Free Field Theories," Phys. Rev. D 10 (1974) 3235.

[29] G. Arutyunov and S. Frolov, "Foundations of the AdS Superstring. Part I," J. Phys. A 42 (2009) 254003 [arXiv:0901.4937 [hep-th]].

[30] T. Klose and T. McLoughlin, "Worldsheet Form Factors in AdS/CFT," Phys. Rev. D 87 (2013) 026004 [arXiv:1208.2020 [hep-th]]. • "Comments on World-Sheet Form Factors in AdS/CFT," J. Phys. A 47 (2014) no.5, 055401 [arXiv:1307.3506 [hep-th]].

[31] Z. Bajnok and R. A. Janik, "String field theory vertex from integrability," JHEP 1504 (2015) 042 doi:10.1007/JHEP04(2015)042 [arXiv:1501.04533 [hep-th]].

[32] V. A. Fateev, "The sigma model (dual) representation for a two-parameter family of integrable quantum field theories," Nucl. Phys. B 473 (1996) 509.

[33] P. Wiegmann, "Exact Factorized S Matrix Of The Chiral Field In Two-dimensions," Phys. Lett. 142B (1984) 173. doi:10.1016/0370-2693(84)91256-5 
[34] L. Castillejo, R. H. Dalitz and F. J. Dyson, "Low's scattering equation for the charged and neutral scalar theories," Phys. Rev. 101 (1956) 453. doi:10.1103/PhysRev.101.453

[35] B. Berg, M. Karowski, P. Weisz and V. Kurak, "Factorized U(n) Symmetric s Matrices in Two-Dimensions," Nucl. Phys. B 134 (1978) 125.

[36] V. Kurak and J. A. Swieca, "Anti-particles As Bound States Of Particles In The Factorized S Matrix Framework," Phys. Lett. B 82 (1979) 289.

[37] B. Berg and P. Weisz, "Exact S Matrix Of The Chiral Invariant Su(n) Thirring Model," Nucl. Phys. B 146 (1978) 205.

[38] E. Abdalla, B. Berg and P. Weisz, "More About The S Matrix Of The Chiral Su(n) Thirring Model," Nucl. Phys. B 157 (1979) 387.

[39] R. Koberle, V. Kurak and J. A. Swieca, "Scattering Theory And 1/n Expansion In The Chiral Gross-neveu Model," Phys. Rev. D 20 (1979) 897 [Erratum-ibid. D 20 (1979) 2638].

[40] A. C. Cubero, "Multiparticle Form Factors of the Principal Chiral Model At Large N," Phys. Rev. D 86 (2012) 025025 doi:10.1103/PhysRevD.86.025025 arXiv:1205.2069 [hep-th]].

[41] P. Orland, "Summing Planar Diagrams by an Integrable Bootstrap," Phys. Rev. D 84 (2011) 105005 doi:10.1103/PhysRevD.84.105005 [arXiv:1108.0058 [hep-th]]. - "Summing Planar Diagrams by an Integrable Bootstrap II," Phys. Rev. D 86 (2012) 045023 doi:10.1103/PhysRevD.86.045023 [arXiv:1205.1763 [hep-th]].

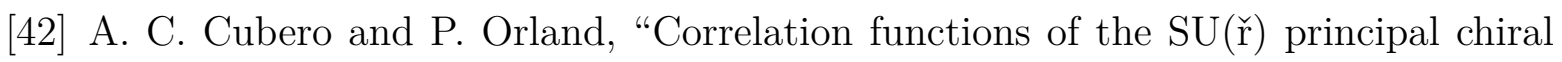
model," Phys. Rev. D 88 (2013) no.2, 025044 doi:10.1103/PhysRevD.88.025044 arXiv:1306.1930 [hep-th]].

[43] A. C. Cubero, "( $2+1$ )-dimensional Yang-Mills theory and form factor perturbation theory," Phys. Rev. D 90 (2014) no.6, 065002 doi:10.1103/PhysRevD.90.065002 arXiv:1405.7639 [hep-th]].

[44] M. Jimbo, H. Konno and T. Miwa, "Massless XXZ model and degeneration of the elliptic algebra $A_{Q, P}\left(\widehat{S L}_{2}\right)$," In *Ascona 1996, Deformation theory and symplectic geometry* 117-138 hep-th/9610079.

[45] E. Ogievetsky, P. Wiegmann and N. Reshetikhin, "The Principal Chiral Field in Two-Dimensions on Classical Lie Algebras: The Bethe Ansatz Solution and Factorized Theory of Scattering," Nucl. Phys. B 280 (1987) 45. doi:10.1016/0550$3213(87) 90138-6$ 
[46] R. A. Janik, "The $\operatorname{AdS}(5) \times \mathrm{S}^{* * 5}$ superstring worldsheet S-matrix and crossing symmetry," Phys. Rev. D 73 (2006) 086006 doi:10.1103/PhysRevD.73.086006 hepth/0603038. 